\title{
UNIFYING THE RESONANT SOLUTIONS OF A BROAD CLASS OF KORTEWEG-DE VRIES EQUATIONS
}

By

Philippe H. Trinh, B.Math.

May 2007

\begin{abstract}
A Thesis
submitted to the School of Graduate Studies and Research

in partial fulfillment of the requirements

for the degree of

Master of Science in Mathematics ${ }^{1}$
\end{abstract}

(C) Copyright 2007

by Philippe H. Trinh, B.Math., Ottawa, Canada

\footnotetext{
${ }^{1}$ The M.Sc. Program is a joint program with the University of Ottawa, administered by the Ottawa-Carleton Institute of Mathematics and Statistics
} 


$\begin{array}{ll}\begin{array}{l}\text { Library and } \\ \text { Archives Canada }\end{array} & \begin{array}{l}\text { Bibliothèque et } \\ \text { Archives Canada }\end{array} \\ \begin{array}{l}\text { Published Heritage } \\ \text { Branch }\end{array} & \begin{array}{l}\text { Direction du } \\ \text { Patrimoine de l'édition }\end{array} \\ \begin{array}{l}\text { 395 Wellington Street } \\ \text { Ottawa ON K1A ON4 }\end{array} & \begin{array}{l}\text { 395, rue Wellington } \\ \text { Ottawa ON K1A ON4 } \\ \text { Canada }\end{array}\end{array}$

Your file Votre référence ISBN: 978-0-494-27026-4 Our file Notre référence ISBN: 978-0-494-27026-4

NOTICE:

The author has granted a nonexclusive license allowing Library and Archives Canada to reproduce, publish, archive, preserve, conserve, communicate to the public by telecommunication or on the Internet, loan, distribute and sell theses worldwide, for commercial or noncommercial purposes, in microform, paper, electronic and/or any other formats.

The author retains copyright ownership and moral rights in this thesis. Neither the thesis nor substantial extracts from it may be printed or otherwise reproduced without the author's permission.
AVIS:

L'auteur a accordé une licence non exclusive permettant à la Bibliothèque et Archives Canada de reproduire, publier, archiver, sauvegarder, conserver, transmettre au public par télécommunication ou par l'Internet, prêter, distribuer et vendre des thèses partout dans le monde, à des fins commerciales ou autres, sur support microforme, papier, électronique et/ou autres formats.

L'auteur conserve la propriété du droit d'auteur et des droits moraux qui protège cette thèse. $\mathrm{Ni}$ la thèse ni des extraits substantiels de celle-ci ne doivent être imprimés ou autrement reproduits sans son autorisation.
In compliance with the Canadian

Privacy Act some supporting forms may have been removed from this thesis.

While these forms may be included in the document page count, their removal does not represent any loss of content from the thesis.
Conformément à la loi canadienne sur la protection de la vie privée, quelques formulaires secondaires ont été enlevés de cette thèse.

Bien que ces formulaires aient inclus dans la pagination, il n'y aura aucun contenu manquant.

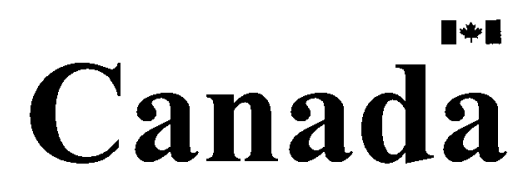




\section{Abstract}

The periodically forced $\mathrm{KdV}$, modified $\mathrm{KdV}(\mathrm{mKdV})$, and extended $\mathrm{KdV}$ (eKdV) equations are considered. Using a method of multiple scales, leading order asymptotic approximations are derived for the steady state solutions near resonance. The parameters and methodology under consideration are inspired by the work of Amundsen, Cox, and Mortell (2006, ZAMP) on the KdV and the problem of resonant sloshing of shallow water in a tank. However, in the case of the $\mathrm{mKdV}$ and $\mathrm{eKdV}$, the addition of a saddle point in the phase plane produces a new array of possible solutions and boundary layer transitions. The connection between the various classes of KdV equations is established and in particular, solutions of the eKdV equation are shown to exhibit behaviour of either the KdV-type or the mKdV-type. Analytic solutions are compared to numerical results and the effectiveness and limitations of the proposed methodology are discussed. 


\section{Acknowledgements}

For the friends life has given me, and for the life friends have given me.

- Loise Wyse ${ }^{2}$

Innocuously tucked away on the fourth floor of the Herzberg Laboratories of Carleton University, is my home away from home. A thank you to the School of Mathematics and Statistics - the bright professors and the amicable administrators - who have all supported me so warmly throughout the years. And as ever, a hearty thank you to my supervisor, Dr. Dave Amundsen, who has taken great delight in joining me while I explore this intimidating world. There is no institution I am more proud to call my home; no adviser I am more proud to call my mentor and confidant.

I am particularly indebted to my wonderful and understanding friends, who, despite my urge to babble on about math, will still smile and nod enthusiastically. Special thanks to Mr. David Ho and Ms. Dorothe Abou-Hamad, who both fought valiantly to keep me young at heart while I vigorously pursued my studies.

Finally, a thank you to my loving family: my mother, Mrs. Lien Hoa Hoang, father, Mr. Tri Dinh Trinh, and sister, Ms. Tu-Quynh Trinh, who tended to, and reminded me of the little things I am prone to forget - eating, sleeping, and staying alive.

\footnotetext{
${ }^{2}$ Dedication in her 1996 book, Women Make the Best Friends.
} 


\section{Dedication}

To my former teachers at Hillcrest High School ${ }^{3}$, Class of 2004 - who would reply to my incessant whining by saying rather grimly,

"You don't know what hard is. But one day, you'll figure it out. And then you'll be sorry."

Thanks for the bold advice. I did figure it out and yes, I'm sorry I did.

\footnotetext{
${ }^{3}$ Hillcrest High School, 1900 Dauphin Road, Ottawa Ontario, K1G 2L7
}

iv 


\section{Contents}

Abstract

Acknowledgements $\quad$ iii

Dedication $\quad$ iv

1 Introduction 1

2 A Review of Korteweg-de Vries Type Equations 4

2.1 The Discovery of the Soliton and the

Derivation of the $\mathrm{KdV} \ldots \ldots \ldots 4$

2.2 The Forced Korteweg-de Vries Equation . . . . . . . . . . . . 7

2.3 The Forced Modified Korteweg-de Vries Equation . . . . . . . . . . . . . . . . . . 8

2.4 The Forced Extended Korteweg-de Vries Equation ......................... 10

2.5 Asymptotic Analysis of the Equations . . . . . . . . . . . . . 11

2.6 Summary . . . . . . . . . . . . . . . . 12

3 Steady Solutions of the

Modified KdV Equation $\quad 13$

3.1 Introduction . . . . . . . . . . . . . . . . 13

3.2 The Non-Dispersive Solutions . . . . . . . . . . . . 14

$\mathrm{V}$ 
3.2.1 Deriving the Non-Dispersive Solutions

Using a Regular Perturbation . . . . . . . . . . . . 15

3.2.2 Existence of Non-Dispersive Solutions . . . . . . . . . . . . 16

3.3 The Equation and its Qualitative Behaviour . . . . . . . . . . . 21

3.3.1 The Phase Plane ............... 21

3.3.2 Energy Analysis and the Quartic Equation . . . . . . . . . . 24

3.4 Asymptotic Analysis of the

Undamped Equation . . . . . . . . . . . . . . . 28

3.4.1 Deriving the Dispersive Solutions . . . . . . . . . . . 30

3.4.2 Avoiding Secularity and the Solvability Condition . . . . . . . 33

3.5 Asymptotic Analysis of the Damped Equation . . . . . . . . . . . 37

3.6 Matching of Solutions . . . . . . . . . . . . . . . 39

3.6.1 Determining $\kappa$ by Enforcing a Matching . . . . . . . . . 39

3.6.2 Determining $\omega_{1}(x)$ and $m(x) \ldots . . . . . . . . . .441$

3.6.3 Determining the Phase Shift $\delta(x) \ldots \ldots . . . . . . .441$

3.6.4 Determining $c$ from the Mean Condition . . . . . . . . . . 42

3.6.5 Summarizing the Parameter Regime . . . . . . . . . . . 43

3.7 Comparison of Analytic Approximations and Numerical Solutions . . 43

3.7 .1 Numerical Solutions . . . . . . . . . . . . . . 44

3.7.2 The Effectiveness of the Derived Approximations . . . . . . 48

3.8 A Novel Approximation to Singular Shocks . . . . . . . . . . . . . 51

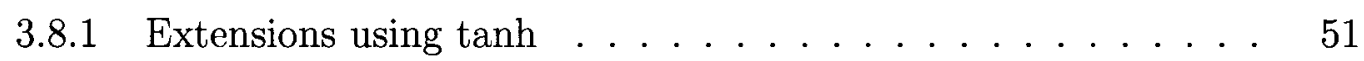

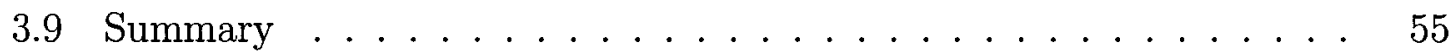

4 Comparing the KdV and mKdV Equations $\quad 57$

4.1 Introduction . . . . . . . . . . . . . 57

4.2 Non-Dispersive Solutions . . . . . . . . . . . . . . . . . 58

4.2.1 A Transformation and the Non-Dispersive Solutions . . . . . . 58

4.2.2 Existence of the Non-Dispersive Solutions and the Resonant Band ................. 59

4.3 The Phase Plane .................... 60

vi 
4.4 The Asymptotic Analysis . . . . . . . . . . . . . . . . . . . 61

4.5 The Numerical Solutions . . . . . . . . . . . . . . . . . 65

4.6 The $\mathrm{mKdV}$ and the $\mathrm{KdV} \ldots \ldots \ldots \ldots \ldots \ldots$

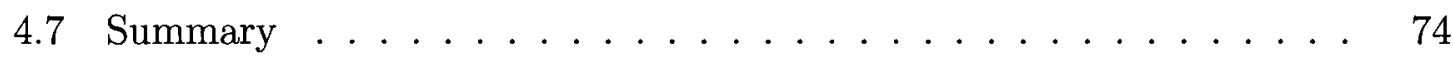

5 The Extended Korteweg-de Vries Equation $\quad 75$

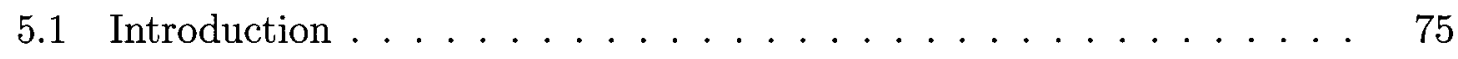

5.2 A Transformation from $\mathrm{eKdV}$ to $\mathrm{mKdV} \ldots \ldots \ldots \ldots . \ldots 77$

5.3 The Transition from KdV to $\mathrm{eKdV} \ldots \ldots \ldots \ldots . \ldots 78$

5.3.1 No Substantial Cubic Effect . . . . . . . . . . . . . . 80

5.3 .2 Substantial Cubic Effect . . . . . . . . . . . . . 83

5.3.3 Predicting the Bifurcation and the Peak Movement . . . . . 88

5.4 In the Middle of the eKdV Spectrum . . . . . . . . . . . 90

5.5 The Transition from eKdV to $\mathrm{mKdV} \ldots \ldots \ldots$. . . . . . . 93

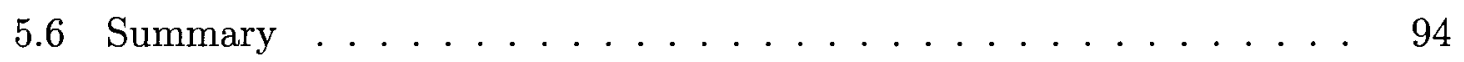

6 Conclusions $\quad 95$

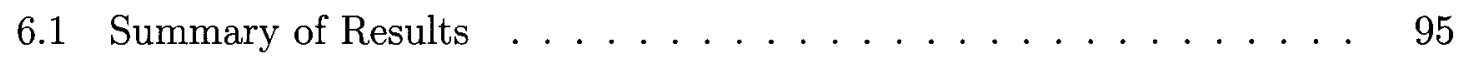

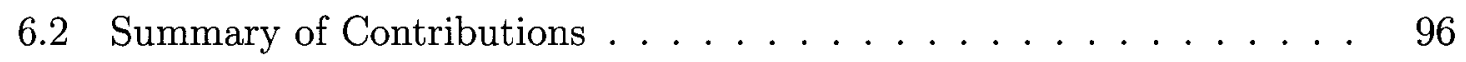

6.3 Future Research . . . . . . . . . . . . . . . . . . . 97

$\begin{array}{ll}\text { Appendices } & 99\end{array}$

A Existence and Uniqueness of Solutions $\quad 99$

$\begin{array}{ll}\text { B Roots of the Quartic Equation } & 101\end{array}$

vii 


\section{Chapter 1}

\section{Introduction}

Just for a moment, close your eyes and imagine a shallow tank, filled with water. The tank is closed at one end, but at the other, you see a piston oscillating periodically, forcing the water to and fro and causing waves to form at the surface. Now imagine the piston pushing at just the right frequency so that resonance is produced, and suppose that the induced oscillations have settled down to final periodic and steady state.

What does the water look like?

The mathematical and experimental origins of this problem begins with the work of Chester and Bones in 1968 ([12], [13]), and much of what we will see in this thesis is inspired, in some way or another, by their profound water-wave problem. The most general aspect of our focus will be the periodically forced extended Korteweg-de Vries $(\mathrm{eKdV})$ equation with viscous damping,

$$
u_{t}-\gamma u_{x x x}+\alpha u u_{x}+\beta u^{2} u_{x}+\Delta u_{x}-\mu u_{x x}=f(x),
$$

where $\gamma, \alpha, \beta, \Delta$, and $\mu$ are real parameters, and $f(x)$ provides the periodic forcing. In 1986, Cox and Mortell showed that for the above water-wave problem, the governing equation found by setting $\beta=0$ in the above expression, an equation known as the forced $\mathrm{KdV}^{1}$. On the other hand, when setting $\alpha=0$, the resulting cubic nonlinear

\footnotetext{
${ }^{\mathbf{1}}$ Formally, the equation is known as the fKdVB, or forced KdV with Burgers' damping.
} 
variant is usually known as the modified Korteweg-de Vries (mKdV) equation which, for example, arises in stratified fluid flow over a topography (see [28]). In this work, all three $K d V$-type variants are considered: the forced $\mathrm{KdV}, \mathrm{mKdV}$, and $\mathrm{eKdV}$.

For the scientist or engineer, these $\mathrm{KdV}$ equations are encountered during many excursions into fluid mechanics or nonlinear waves. Their immense ubiquity and tendency to appear in fluid flow phenomena have made them into veritable celebrities, gracing the cover of hundreds of research articles.

But these KdV-type equations are also of great importance for the mathematician: They are fascinating case studies into the various mathematical techniques available to applied researchers. The equations hide a wealth of truly rich intricacies and many of the methods used in studying these problems have gone on to inspire or complement research in other important equations, such as the Nonlinear Schrödinger and the Sine-Gordon equations, to name a few.

The type of solutions which arise is highly dependent on the particular choice of parameters; certain choices are more amenable to standard analysis, while other incarnations are more difficult or devious. We will first study the $\mathrm{KdV}, \mathrm{mKdV}$, and eKdV where the parameters corresponding to the dispersion $(\gamma)$ and damping $(\mu)$ are small, while the forcing and nonlinear effects $(\alpha$ and $\beta$ ) are of primary importance. However, we will later bridge together the three classes by studying the effects of varying $\alpha$ and $\beta$. In this fashion, our work captures a wide range of relevant physical parameters.

But for these forced KdV variants, there are no closed form solutions and certainly no exact methods. Here, approximate methods such as those using the method of multiple scales or methods of averaging are often needed in order to derive asymptotic expansions. These expansions provide the means of approximating the solutions at usually an excellent level of accuracy.

The purpose of this thesis is twofold. The most immediate goal is to present a detailed analytic theory of the three types of equations mentioned thus far: the $\mathrm{KdV}, \mathrm{mKdV}$, and $\mathrm{eKdV}$. The culmination of this portion of our work will be a general methodology which will allow researchers to derive leading order asymptotic solutions, not only these KdV-type equations, but hopefully to an even broader class 
of problems.

The second and perhaps more important purpose is to unify these three archetypal KdV-type equations and provide a more global perspective of the problem at hand. Although many individual studies of the $\mathrm{KdV}, \mathrm{mKdV}$, or eKdV exist, there are few (if any) that have sought to compare and contrast these three equations under the same heading. Our work in this respect is distinct and revealing.

In Chapter 2, we begin by introducing the reader to some of the countless applications of KdV-type equations and more importantly, review the current state of the literature and summarize some of the key papers which have motivated and inspired our research.

The bulk of our work however, begins in Chapter 3 where we study the forced $\mathrm{mKdV}$ equation and provide the core methodology for which much of the later text refers to. Chapter 4 , where we compare the $\mathrm{mKdV}$ and $\mathrm{KdV}$ equations provides a natural link from our new work with that of Amundsen, Cox, and Mortell [2] on the forced KdV, a motivating work in our research (to be reviewed in Chapter 2). Finally, the fruits of our labour are applied to the most general of these KdV-type equations in Chapter 5 where we examine the Extended KdV equation in detail, leveraging our results from the $\mathrm{mKdV}$ and $\mathrm{KdV}$ equations. 


\section{Chapter 2}

\section{A Review of Korteweg-de Vries Type Equations}

\subsection{The Discovery of the Soliton and the Derivation of the $\mathrm{KdV}$}

Any introduction to the celebrated Korteweg-de Vries equation would be sorely incomplete without reference to the famous observation by the Scottish engineer, John Scott Russell, as he traveled by horseback along the Edinburgh-Glasgow canal in 1834 [36]:

I was observing the motion of a boat which was rapidly drawn along a narrow channel by a pair of horses, when the boat suddenly stopped not so the mass of water in the channel which it had put in motion; it accumulated round the prow of the vessel in a state of violent agitation, then suddenly leaving it behind, rolled forward with great velocity, assuming the form of a large solitary elevation, a rounded, smooth and well-defined heap of water, which continued its course along the channel apparently without change of form or diminution of speed. I followed it on horseback, and overtook it still rolling on at a rate of some eight or nine miles an hour, preserving its original figure some thirty feet long and a foot to a 
foot and a half in height. Its height gradually diminished, and after a chase of one or two miles I lost it in the windings of the channel.

The phenomenon was so unusual and remarkable in appearance that Russell immediately recognized its significance and claimed,

This is a most beautiful and extraordinary phenomenon: the first day I saw it was the happiest day of my life. Nobody had ever had the good fortune to see it before or, at all events, to know what it meant.

He would later call it his "great waves of translation", and would devote much of his later life studying the problem. Both Boussinesq (in 1871) [8] and Lord Rayleigh (in 1876) [35] would later contribute significantly to the theory, but it was not until 1895 that an equation was provided. Korteweg and de Vries [24] provided the expression for the phenomenon, which they wrote as,

$$
u_{t}=\frac{3}{2}\left(\frac{g}{h}\right)^{1 / 3}\left(u u_{x}+\frac{1}{3} \sigma u_{x x x}\right)
$$

where $h$ is the undisturbed depth of the water, $g$ is the acceleration due to gravity, $\sigma$ incorporates the surface tension, and $u(x, t)$ is the wave profile above the undisturbed depth. Placed in standard form, this equation is,

$$
u_{t}-6 u u_{x}+u_{x x x}=0,
$$

the canonical KdV equation. Zabusky and Kruskal [42] later showed in 1965 that nonlinear waves, such as the ones for the $\mathrm{KdV}$, can interact strongly and continue thereafter almost as if there was no interaction at all (see figure 1). This observation of usually localized and solitary behaviour would lead them to coin the name 'soliton' in labeling these special solutions. And soon after this discovery, research into the $\mathrm{KdV}$ and similar nonlinear equations continued at a frenzied pace.

The $\mathrm{KdV}$ is considered as one of the simplest nonlinear differential equations to write down. But those brave enough to first attempt an analytical study of the equation are in for a rude awakening: closed form solutions of the KdV are not easy to find, and researchers have had to work long and hard until several new techniques 

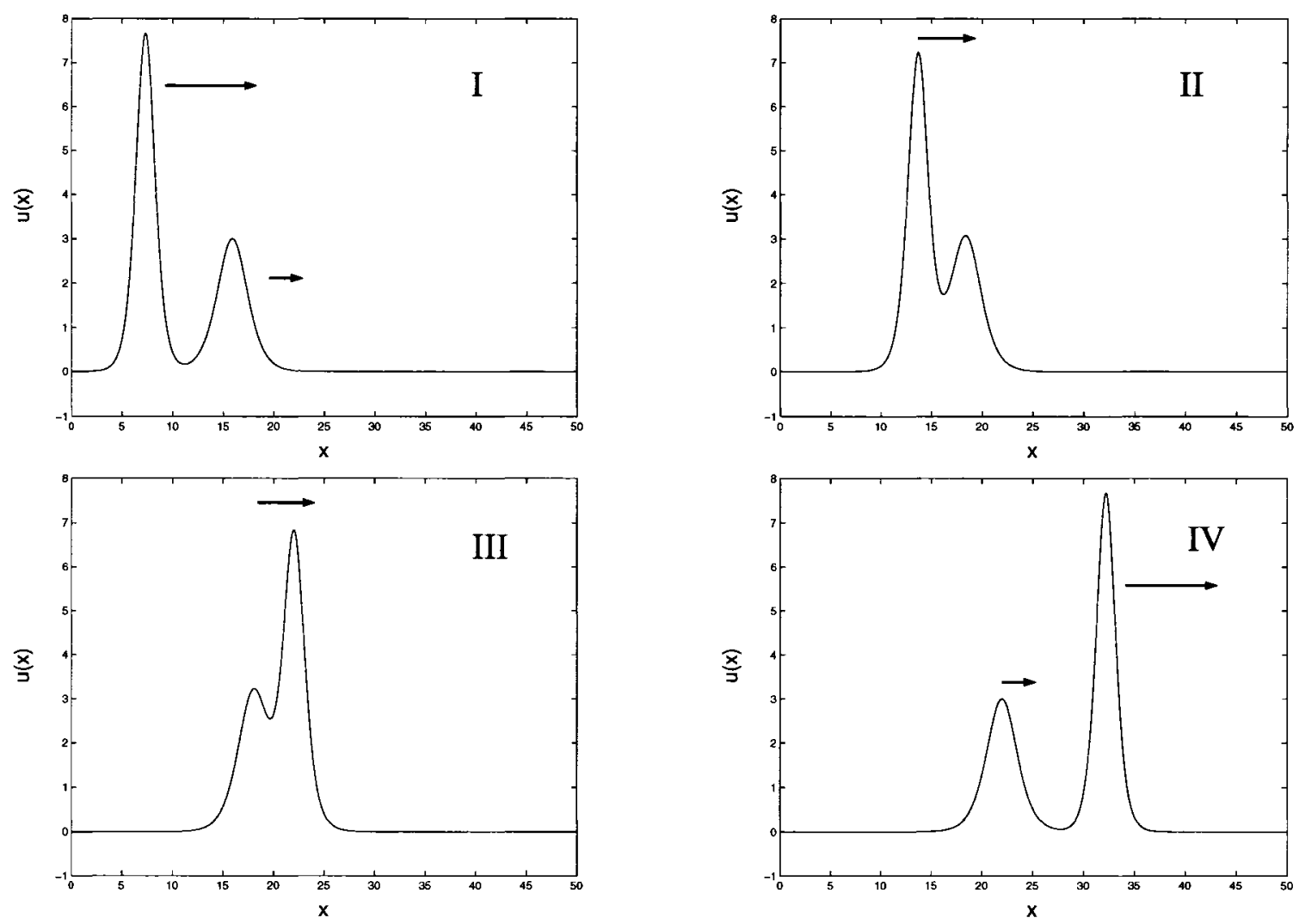

Figure 1: A sequence depicting the interaction of two 'solitons' 
were developed that could handle such equations (see, for example details of the Inverse Scattering Transform in [16]).

Our investigation into KdV-type equations will be confined to a specific type of variation which has been largely motivated by the work of Chester and Bones on the oscillations of shallow water in a tank.

\subsection{The Forced Korteweg-de Vries Equation}

In the introduction of this thesis, we presented the problem originally proposed by Chester in his 1967 paper [12]: How does a fluid behave when confined to a shallow tank closed at one end by a rigid barrier and at the other by a periodically vibrating piston? In his work, Chester began with the two-dimensional Navier-Stokes equations for an incompressible fluid and derived a steady-state equation which governed the periodic oscillations of the water surface. Shortly after the mathematical formulation, Chester and Bones presented a comparison between numerical solutions of the model and experimental observations in [13] (see figure 2), performed at the Mechanical Engineering laboratory of Bristol University. The agreement between theory and experiment was confirmed.

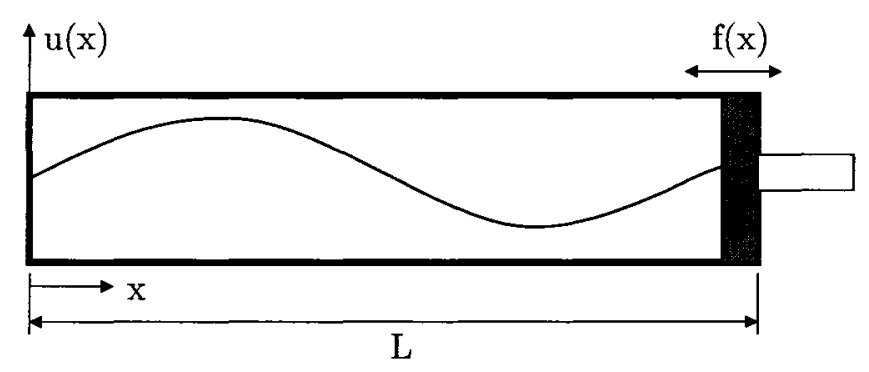

Figure 2: A sketch of the tank considered by Chester and Bones. The water in the tank is being forced at one end by a vibrating piston. (Chester and Bones [12] [13])

In 1986, Cox and Mortell [14] provided an alternate expression to Chester's equation, modeled as a forced KdV equation with Burgers' type damping, 


$$
u_{t}-\gamma u_{x x x}+\alpha u u_{x}-\mu u_{x x}=f(x),
$$

and this form of the water-wave problem will be used throughout this thesis.

However, deriving the approximate steady state, resonant solutions to the forced $\mathrm{KdV}$ equation above is a rather non-trivial problem. Research began with a paper by Ockendon and Ockendon [32] in 1972, where asymptotic solutions to a modest set of solutions were provided. This was continued in 1986 by Ockendon, Ockendon, and Johnson [33], when the analysis was extended to a wider range of solutions.

More recently in [2], Amundsen, Cox, and Mortell were able to extend the methodology and construct asymptotic solutions to the entire resonant band and in particular studied some of the more complex solutions avoided by previous authors. The approach and interpretation found in their paper (and also in [4]) is the principle motivation behind our own analysis in this work. In fact, we will review much of their work during our discussion of the KdV in Chapter 4.

\subsection{The Forced Modified Korteweg-de Vries Equation}

The modified Korteweg-de Vries Equation (mKdV) results when we replace the quadratic nonlinearity in the KdV with a cubic nonlinearity, such as in,

$$
u_{t}-\gamma u_{x x x}+\beta u^{2} u_{x}-\mu u_{x x}=f(x),
$$

Unfortunately, we shall later see that this rather innocent change produces qualitatively different solutions in comparison with the regular $\mathrm{KdV}$. The $\mathrm{mKdV}$ equation arises in weakly dispersive nonlinear wave motion where, for example, symmetry considerations of the media or boundary conditions requires the quadratic nonlinearity of the KdV to vanish. This may occur in interfacial gravity (or capillary gravity) waves between two superposed, finite layers of immiscible fluids of different densities [34]. In these and other cases, the mKdV may arise as the leading order equation. 
We are solely interested in steady solutions of the mKdV equation, thus setting $u_{t}=0$ and integrating yields,

$$
-\gamma u_{x x}+\frac{\beta}{3} u^{3}-\mu u_{x}=F(x)+c,
$$

where $F(x)+c$ is an anti-derivative of the forcing $f(x)$. This oft-encountered equation found in the theory of ordinary differential equations is a variant of the well-known Duffing Equation, which has the physical interpretation of describing the motion of a damped oscillator with a more complicated potential than in simple harmonic motion. Figure 3 illustrates the phase plane of one of the many Duffing-type equations, this one with a centre and two saddle fixed points.

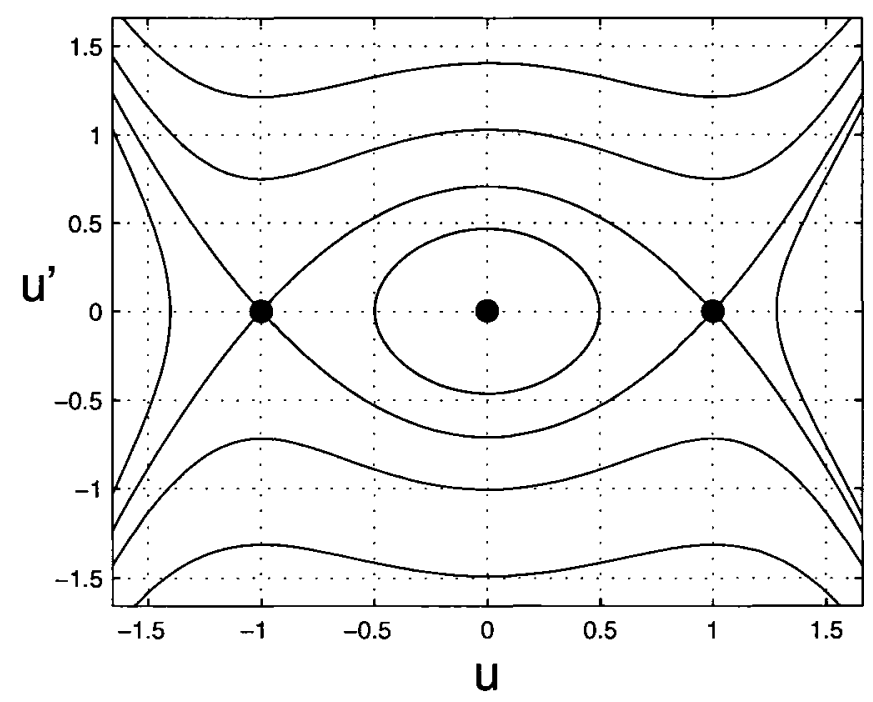

Figure 3: Phase plane of the Duffing/Pendulum problem, $u^{\prime \prime}=u^{3}-u$

Over the years, many variants of the Duffing equation have surfaced and although the equation possesses no closed form solutions, different approximation methods have been developed depending on the parameters involved. Perhaps the most natural and first variant studied was a weakly nonlinear $(\beta \ll 1)$ and unforced variation 
$(F(x)+c \equiv 0)$. This type of equation is almost a classic problem found in countless texts on perturbation theory or nonlinear oscillation theory (See [19], [21], [39], and [23]).

From here, different Duffing variants can be found in the literature with different methods used in analyzing the behaviour. For example, Kaper uses a method of multiple scales to study the weakly forced Duffing equations in [22], Struble and Yionoulis use an asymptotic expansion in circular functions to examine a weakly forced and weakly nonlinear Duffing, and Elias-Zuniga applies a harmonic balance method to the Duffing equation using elliptic functions in [43]. The closest and most revealing work to what we will do in this thesis is attributed to Kuzmak, who derives in [25] the leading order asymptotic solutions to an unforced Duffing equation with slowly varying coefficients. Kuzmak's analysis is quite important to our approach, and we will briefly mention his name throughout the text.

However, even with so much work already done on the Duffing equation, our work here is distinct and in many ways, an extension of previous work on the topic. We will instead focus on a Duffing Equation where the leading order effects are both from the cubic nonlinearity $\beta$ and the forcing $f(x)$.

\subsection{The Forced Extended Korteweg-de Vries Equation}

As we mentioned earlier, the KdV equation arises in the description of weakly nonlinear, long wavelength water waves when second order nonlinear and dispersive terms are retained (see Whitham [41]). But if we instead retain terms of third order in wave amplitude, the result is the Extended Korteweg-de Vries equation, or eKdV for short.

One of the first applications of an eKdV equation with forcing was studied by Meville and Helfrich [29] in the context of transcritical flow of a two-layer fluid over a bottom topography. Nearly in parallel, Grimshaw, Smyth, and Marchant (See [20], [28], [37]) derived and studied a similar forced eKdV equation, also to study the resonant flow of a fluid over a topography. 
As an example of the eKdV, consider equation (3.8) of the paper by Marchant and Smyth [28],

$$
-u_{t}+u_{x x x}-\alpha c_{1} u^{2} u_{x}+6 u u_{x}-\Delta u_{x}=-\left(1+\alpha c_{8} \Delta\right) G_{x}(x),
$$

where $\alpha$ is a relative measure of the typical wave amplitude, $c_{1}$ and $c_{8}$ are constants which depend on the density stratification, and $\Delta$ is a detuning parameter. Instead of the periodic oscillator generating the waves of a tank we presented for the forced $\mathrm{KdV}$, this time the forcing $G(x)$ is localized and is a measure of the bottom topography over which the water flows (See Equation 3.1 in their paper).

However, one of the weaknesses of the work by Marchant and Smyth is the fact that they largely neglect the effects of the cubic nonlinearity - an assumption which essentially allows them to leverage the quadratic KdV theory in understanding the eKdV. This was also emphasized in a comparison by Melville and Helfrich in [29],

But what happens if the cubic nonlinearity is of equal importance to the quadratic nonlinearity? This question is certainly of physical importance in some applications and is of course a mathematically interesting question in its own right.

Our research on the eKdV in Chapter 5 will focus on leveraging the $\mathrm{mKdV}$ and $\mathrm{KdV}$ theory developed beforehand to study the $\mathrm{eKdV}$ for varying degrees of quadratic and cubic nonlinearities. In particular, we will examine the effects of perturbing an $\mathrm{mKdV}$ with a quadratic nonlinearity ( $\mathrm{mKdV}$ to eKdV transition), the effects of perturbing a $\mathrm{KdV}$ with a cubic nonlinearity ( $\mathrm{KdV}$ to $\mathrm{KdV}$ transition), and how the solutions behave when both cubic and quadratic regimes are in effect ('fully' eKdV).

\subsection{Asymptotic Analysis of the Equations}

For the most part, our asymptotic analysis in the chapters to come are stand-alone and can be understood with only an introductory knowledge of perturbation theory. As we have mentioned previously, our methodology follows closely with the work of Amundsen, Cox, and Mortell during their study of the KdV in [2].

It appears that this special technique using the method of multiple scales originated with Kuzmak in his 1959 paper [25]. Luke also contributed to the analytical 
theory in his 1965 work [26], and thus, the key ideas behind the method is referred to by some authors (such as [23]) as the Kuzmak-Luke method of multiple scales.

\subsection{Summary}

- The KdV equation is ubiquitous in nature and is the simplest equation which incorporates both nonlinearity and dispersion.

- We will be focused on three variants of the $\mathrm{KdV}$ : (1) KdV with quadratic nonlinearity, (2) Modified KdV (mKdV) with cubic nonlinearity, and (3) Extended $\mathrm{KdV}(\mathrm{eKdV})$ with both quadratic and cubic nonlinearity.

- Our research is motivated by the application of the KdV to shallow water in a tank by Chester and Bones ([12] and [13]). In particular, we are interested in the resonant behaviour of strongly forced KdV-type equations with both leading order forcing and nonlinearity. 


\section{Chapter 3}

\section{Steady Solutions of the Modified KdV Equation}

\subsection{Introduction}

We begin our analysis of KdV-type equations with the forced modified Korteweg-de Vries (mKdV) equation with Burgers' damping,

$$
u_{t}-\gamma u_{x x x}+\Delta u_{x}+\beta u^{2} u_{x}-\mu u_{x x}=f(x)
$$

The terms, which are later explained in more detail, represent the effects of dispersion, nonlinearity, a detuning from resonance, damping, and an external forcing. We will confine ourselves to the steady solutions of the forced $\mathrm{mKdV}$ in (8), and thus setting $u_{t}=0$, the equation becomes,

$$
-\gamma u_{x x x}+\Delta u_{x}+\beta u^{2} u_{x}-\mu u_{x x}=f(x),
$$

which can then be integrated to yield,

$$
-\gamma u_{x x}+\frac{\beta}{3} u^{3}+\Delta u-\mu u_{x}=F(x)+c
$$

where we have defined $F(x)+c$ as an anti-derivative of $f(x)$. As we alluded to during our review of the $\mathrm{mKdV}$ equation in Chapter 2, this equation is simply one of the 
many variants of the well known Duffing equation. But our problem is unique by virtue of the fact that we consider both the effects of leading order non-linearity and forcing. With these two dominant effects in play, the solutions and the methodology differs from previous work on the Duffing equation (see for example, [21], [19], and $[38])$.

The existence and uniqueness of solutions of (9) will be established implicitly as we construct the leading order asymptotic solutions. And while not within the scope of this work, we also briefly discuss the details of existence and uniqueness in Appendix A.

Our plan of action for this chapter will be as follows. Much of the essential groundwork will be laid in Sections 3.2 and 3.3, where we study the mKdV equation broadly and qualitatively, and establish simple, yet limited solutions. This will illuminate our extensive asymptotic analysis to the undamped mKdV equation in Section (3.4), which will soon after be extended to including damping in Section 3.5. Finally, we will discuss the parameter selection (the so-called matching conditions) in Section 3.6, before comparing our analytical approximations to numerical solutions of the equation in Section 3.7.

Perhaps the most exciting result of the work in this portion of our work will be found in Chapter 5, where we study the extended Korteweg-de Vries equation, which contains both quadratic and cubic nonlinearities. In fact, we shall see that a clear understanding of the mKdV reveals much about the seemingly intimidating and unapproachable $\mathrm{eKdV}$ equation.

\subsection{The Non-Dispersive Solutions}

Our asymptotic analysis will proceed with the following strongly nonlinear and strongly forced modified Korteweg-de Vries equation with Burger's damping,

\begin{tabular}{c} 
Forced mKdV \\
$-\underbrace{\gamma u_{x x x}}_{\text {dispersion }}+\underbrace{\Delta u_{x}}_{\text {detuning }}+\underbrace{\frac{\beta}{3} u^{2} u_{x}}_{\text {cubic nonlinearity }}-\underbrace{\mu u_{x x}}_{\text {damping }}=\underbrace{f(x)}_{\text {forcing }}$ \\
\hline
\end{tabular}


where we define $x$ over the interval $[0,2 L]$. The parameter $\gamma$ represents the dispersion, $\Delta$ the detuning from resonance, $\beta$ the degree of nonlinearity, and $\mu$ the damping. To be consistent with physical models, we assume that both the dispersion, $\gamma$ and the damping $\mu$ are small and of the same order. The forcing $f(x)$ is assumed to be smooth, $2 L$ periodic, and with zero-mean. Finally, we impose a zero mean condition on the solutions,

$$
\int_{0}^{2 L} u(x, t) d x=0 .
$$

This mean condition is a key constraint in the problem, limiting the types of solutions that may occur. The constraint is a physically motivated condition, corresponding, for example, to conservation of mass in the system.

\subsubsection{Deriving the Non-Dispersive Solutions Using a Regular Perturbation}

Integrating equation (11) yields,

$$
\gamma u_{x x}+\mu u_{x}=\frac{\beta}{3} u^{3}+\Delta u-(F(x)+c),
$$

where $F(x)+c$ is an anti-derivative of the forcing, $f(x)$. Since we are assuming both $\gamma$ and $\mu$ are small, we can first try a typical perturbation expansion in powers of $\sqrt{\gamma}$ : Let us expand,

$$
u(x)=u_{0}(x)+\sqrt{\gamma} u_{1}(x)+\gamma u_{2}(x)+\ldots
$$

Using this expansion in equation (13) yields the leading order equation,

$$
\mathcal{O}(1): \frac{\beta}{3} u_{0}^{3}+\Delta u_{0}-(F(x)+c)=0 .
$$

Thus the leading order non-dispersive solution will be one of the solutions of the cubic equation (14): 


$$
\begin{gathered}
\text { Non-Dispersive Solutions } \\
u_{1,2,3} \equiv \frac{\rho^{1 / 3}}{2 \beta} e^{2 \pi i k}-\frac{2 \Delta}{\rho^{1 / 3}} e^{-2 \pi i k}, \quad k=0,1,2
\end{gathered}
$$

where,

$$
\rho=(12[F(x)+c]+4 \sqrt{M}) \beta^{2}, \quad M=\frac{4 \Delta^{3}+9 \beta[F(x)+c]^{2}}{\beta}
$$

But of course, to be valid leading approximations, these equations would not only have to be well defined, but would also need to satisfy the condition of zero mean (12). The question of where these non-dispersive solutions $u_{1,2,3}$ are valid and where they are not are studied in the next section.

\subsubsection{Existence of Non-Dispersive Solutions}

A natural question to ask is why the non-dispersive solutions $u_{1,2,3}$ of equation (15) would satisfy the zero-mean condition for some values of $\Delta$, but fail for others? The reason can be seen through simple graphical arguments. To repeat, the non-dispersive solutions are the cubic roots of equation (14),

$$
G(u) \equiv \frac{\beta}{3} u^{3}+\Delta u-C=0
$$

where we have defined $C \equiv F(x)+c$, and have chosen to represent the cubic polynomial (in $u$ ) for fixed forcing by $G(u)$. As shown previously, the non-dispersive solutions are,

$$
u_{1,2,3} \equiv \frac{\rho^{1 / 3}}{2 \beta} e^{2 \pi i k}-\frac{2 \Delta}{\rho^{1 / 3}} e^{-2 \pi i k}, \quad k=0,1,2
$$

where,

$$
\rho=(12[F(x)+c]+4 \sqrt{M}) \beta^{2}, \quad M=\frac{4 \Delta^{3}+9 \beta[F(x)+c]^{2}}{\beta} .
$$

We remind the reader that the cube root $\rho^{1 / 3}$, refers to the principal branch, with the other two branches provided by $k=1$ and $k=2$. Of particular interest are two 
points on each of the three solutions: The point $\Delta^{*}$ for which $\Delta=0$, and the point $\Delta=\Delta_{M}$ for which $M=0$.

- $\Delta^{*}=0:$ From one solution to three

Let us begin by examining Figure 4, which illustrates the cubic $G(u)$ for $\Delta \gg 0$ at three values of the forcing: At the minimum $C=C_{\min }$, at the maximum $C=C_{\max }$, and for no perturbation $C=0$.

The choice of $\Delta>0$ has the effect of allowing only a single real root of $G(u)$ over the entire variation of the forcing. As the forcing varies, the cubic root shifts and this produces the non-dispersive solution $u_{1}$ shown in Figure 5. Clearly, it satisfies the zero-mean condition so long as the forcing is symmetric.

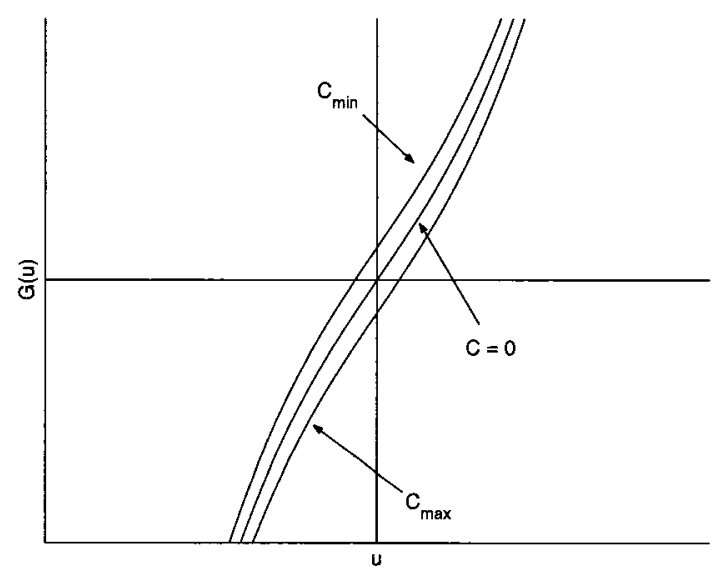

Figure 4: Shifting the curve up and down produces the three non-dispersive solutions. Note the existence of only one real root.

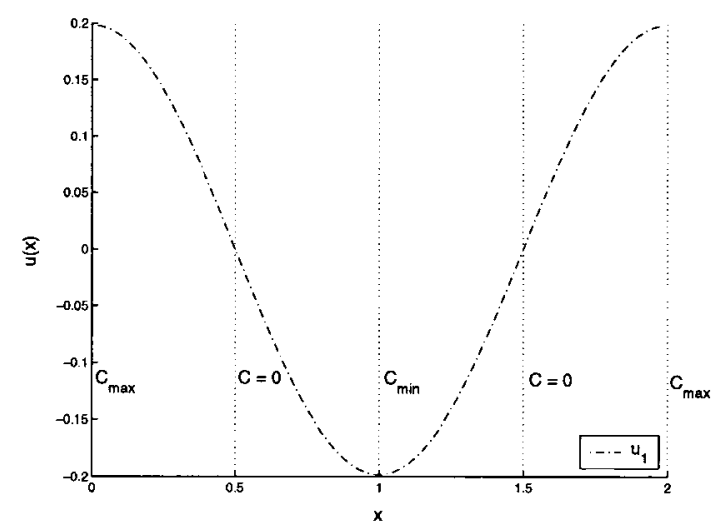

Figure 5: Corresponding non-dispersive solution that exists everywhere.

However, as $\Delta$ tends to 0 , an inflection point begins to form on the cubic $G(u)$ as seen in Figure 6. This has the effect of producing regions of large increase and decrease on the non-dispersive solution $u_{1}$, as in Figure 7.

Past $\Delta=0$, the lone root splits, separating into three real roots (Figure 8), and this critical value of $\Delta$ we define as,

$$
\Delta^{*}=0 .
$$




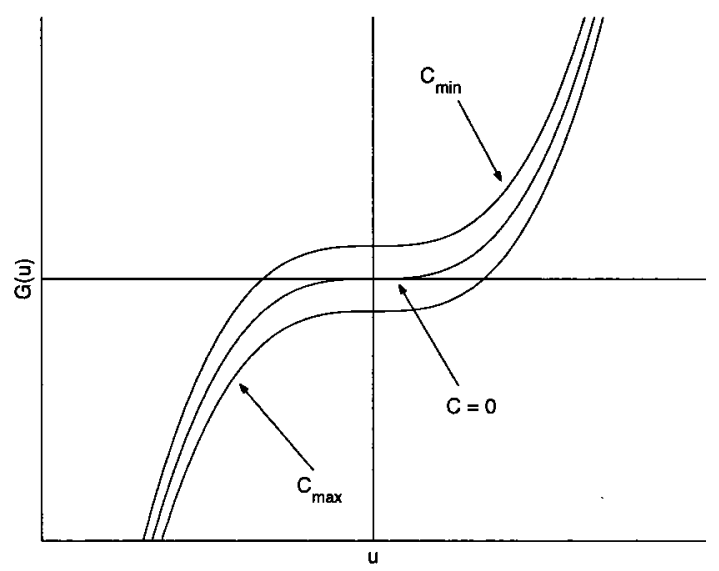

Figure 6: Shifting the curve up and down produces the three non-dispersive solutions. Notice the large change in the cubic root as the curve shifts.

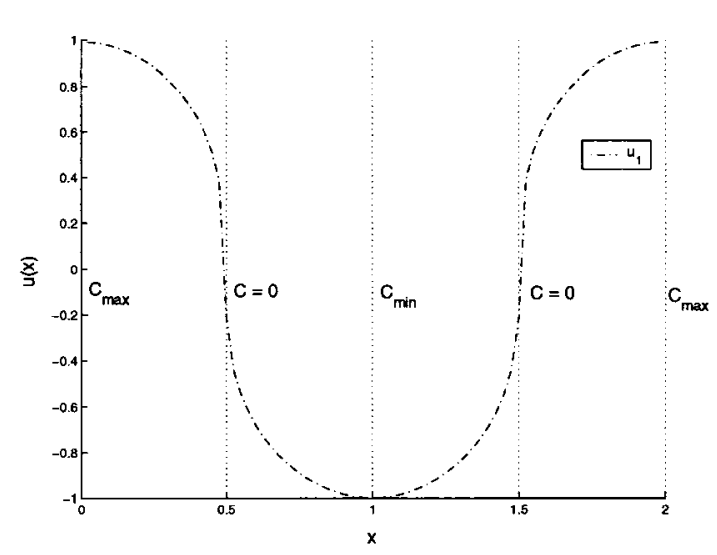

Figure 7: Corresponding non-dispersive solution that exists everywhere. Notice the beginning formation of a vertical tangent.

Also note that while three solutions are clearly visible in Figure 9, only the centre root, $u_{3}$ satisfies the mean condition.

\section{- $\Delta_{M}^{*}$ : The creation of a cusp}

Upon examination of Figure 8, we notice an alarming problem: If the maximum (or minimum) of $G(u)$ is not sufficiently large (or small), two of the cubic roots may be complex valued for certain values of the forcing. Clearly, these critical points can be found by studying the maxima and minima of $G(u)$ at $C=0$, which are found at,

$$
u_{\min / \max }= \pm \sqrt{\frac{-\Delta}{\beta}}
$$

with corresponding height,

$$
G\left(u_{\min / \max }\right)= \pm \sqrt{\frac{-\Delta}{\beta}}\left(\frac{2 \Delta}{3}\right)
$$

There is a precise point $x$, corresponding to the forcing $C \equiv F(x)+c$ when the 


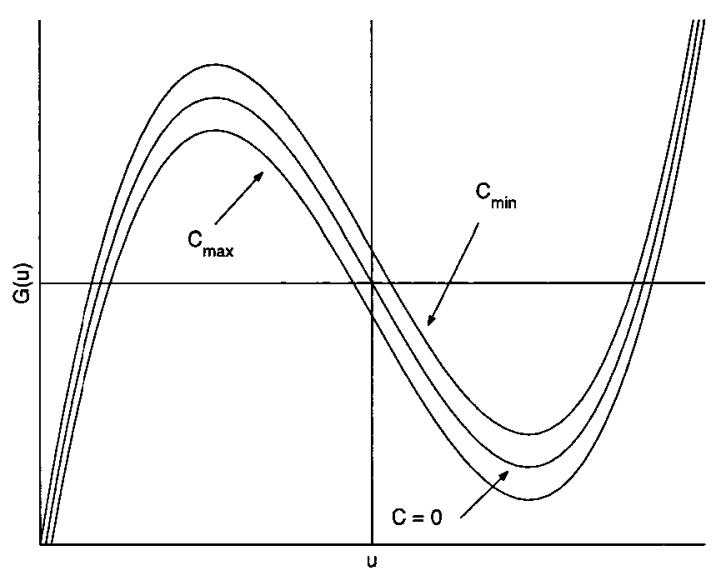

Figure 8: Shifting the curve up and down produces the three non-dispersive solutions. Note the existence of three real roots everywhere.

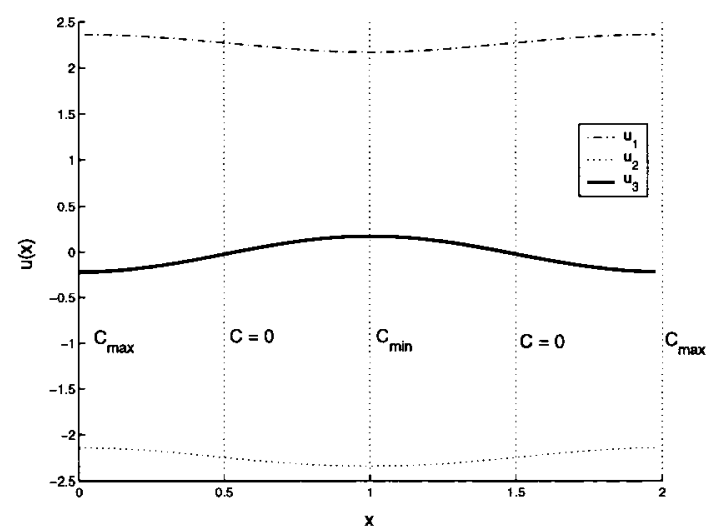

Figure 9: Corresponding non-dispersive solutions that exist everywhere.

cubic $G(u)$ is on the verge of losing a root (via a double root), i.e. when the height (18) is equal to $-C$,

$$
\sqrt{\frac{-\Delta}{\beta}}\left(\frac{2 \Delta}{3}\right)=-C
$$

Now solving for $\Delta$ yields,

$$
\Delta_{M} \equiv\left(-\frac{9}{4} \beta[F(x)+c]^{2}\right)^{1 / 3}
$$

The label $\Delta_{M}$ is appropriate as setting $M=0$ in the non-dispersive solutions also yields this value of $\Delta$. The point we are interested here is when the forcing $(F(x)+c)^{2}$ is at a maximum and thus the corresponding $\Delta_{M}$ is the smallest point in which the solutions $u_{1,2,3}$ with $\Delta<\Delta_{M}^{*}$ first forms a cusp. That is,

$$
\Delta_{M}^{*}=\left(-\frac{9}{4} \beta \max _{x \in[0,2 L]}\left\{(F(x)+c)^{2}\right\}\right)^{1 / 3}
$$

Figure 10 illustrates the cubic $G(u)$ when $\Delta=\Delta_{M}^{*}$ and clearly shows the birth 
of a cusp when $C^{2}$ is maximal. The non-dispersive solutions for this value of $\Delta$ contain two (and in general, potentially three) cusps, as shown in Figure 11.

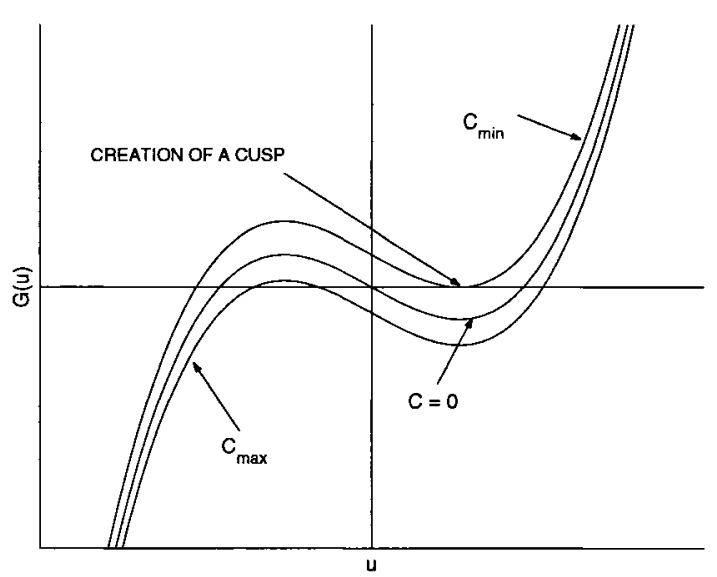

Figure 10: The birth of a cusp due to the cubic equations.

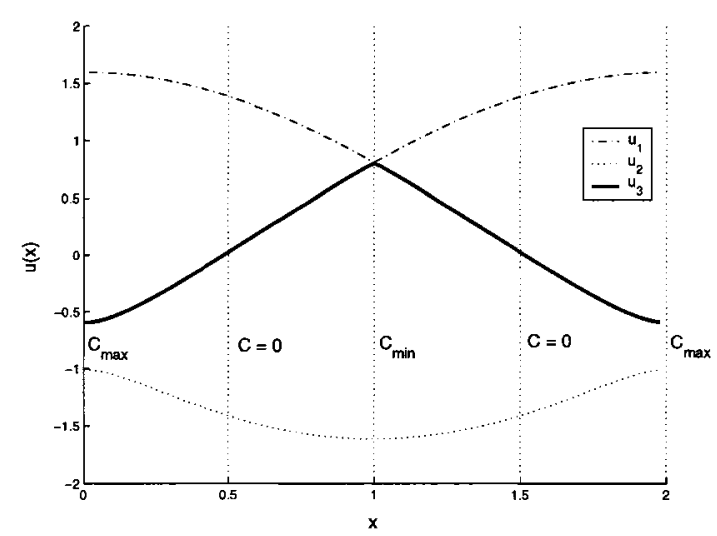

Figure 11: Corresponding nondispersive solutions. Here, two solutions have a cusp.

\section{- $\Delta_{M}^{*} \leq \Delta \leq \Delta^{*}$ : The resonant band}

With this in mind, we have successfully delimited a band of $\Delta$ values where all three non-dispersive solutions fail to be real-valued throughout the entire variation of the forcing: We call the band $\Delta_{M}^{*} \leq \Delta \leq \Delta^{*}$, the resonant band, and it corresponds to a location where the non-dispersive solutions can no longer satisfy the $\mathrm{mKdV}$ equation alone.

As an interesting illustration of our classification, examine Figure 12, which is the cubic arrangement corresponding to Figure 13, which depicts the three nondispersive solutions, $u_{1,2,3}$ within the resonant band, $\Delta_{M}^{*} \leq \Delta \leq \Delta_{\rho}^{*}$. We have taken particular care to only plot the solutions at points where the imaginary components are zero.

As depicted, $u_{1}$ does not exist in the centre (region III), $u_{2}$ does not exist in the edges (regions I and $\mathrm{V}$ ), and $u_{3}$ does not exist in either the edges or the centre (regions I, III, and V). The size of the regions II and IV where three solutions exist simultaneously is a function of the size of the $\max / \mathrm{min}$ of the unperturbed 
cubic in Figure 12.

It is quite amazing then, that a smooth solution of the $\mathrm{mKdV}$ can be pieced together using combinations of these non-dispersive solutions. Most likely, the reader can already predict the outcome: A solution will follow $u_{1}$ in region I, only to jump down to the bottom $u_{2}$ solution, using the transition layer II, where all three non-dispersive solutions are valid. Similarly, the solution will follow $u_{2}$ in region III, then jump back up to $u_{1}$ through region IV.

Thus our focus turns now to the resonant band, where the non-dispersive solutions alone cannot satisfy the mKdV equation. We summarize our findings in the following table:

\begin{tabular}{|c|c|c|}
\hline$\Delta<\Delta_{M}^{*}$ & $\Delta_{M}^{*} \leq \Delta \leq 0$ & $\Delta>0$ \\
(Non-Dispersive Regime) & (Resonant Band) & (Non-Dispersive Regime) \\
\hline \hline Three roots & One or three roots & One root \\
$u_{1}, u_{2}, u_{3}$ exists everywhere & $u_{1}, u_{2}, u_{3}$ exists but & $u_{1}$ satisfies mean condition \\
$u_{3}$ satisfies mean condition & only for limited ranges & \\
\hline
\end{tabular}

\subsection{The Equation and its Qualitative Behaviour}

In the previous section, we derived the leading order non-dispersive solutions and more importantly, discovered a region (the resonant band) in which the non-dispersive solutions alone could not satisfy the mKdV equation. Before proceeding to an asymptotic analysis of the resonant band, we will analyze some the qualitative aspects of the $\mathrm{mKdV}$ in detail.

\subsubsection{The Phase Plane}

We can turn to an analysis of the phase plane of equation (13) in the undamped case for inspiration. For fixed values of $x$, corresponding to 'snapshots' of our solution, the result can be sketched in a two-dimensional phase plane. So if we let $C \equiv F(x)+c$ and $\mu=0$, equation 13 yields, 


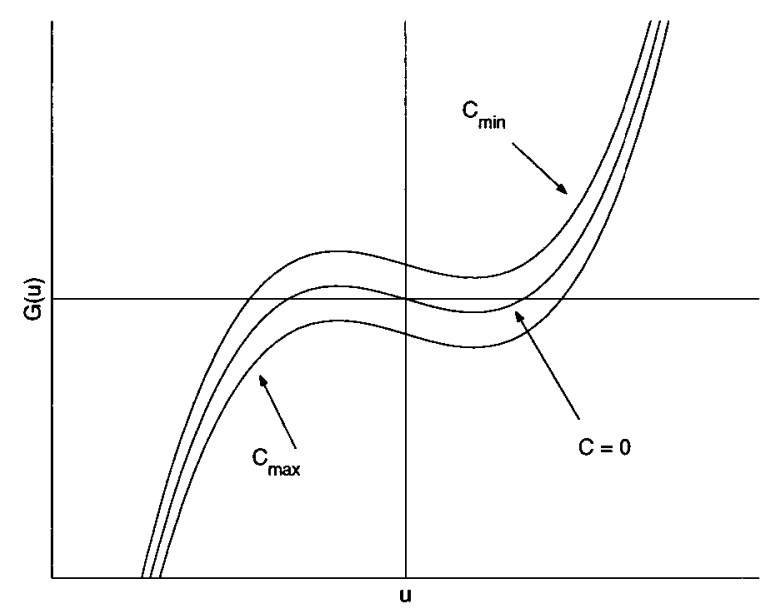

Figure 12: Cubic arrangement within the resonant band, $\Delta_{M}^{*} \leq \Delta \leq 0$

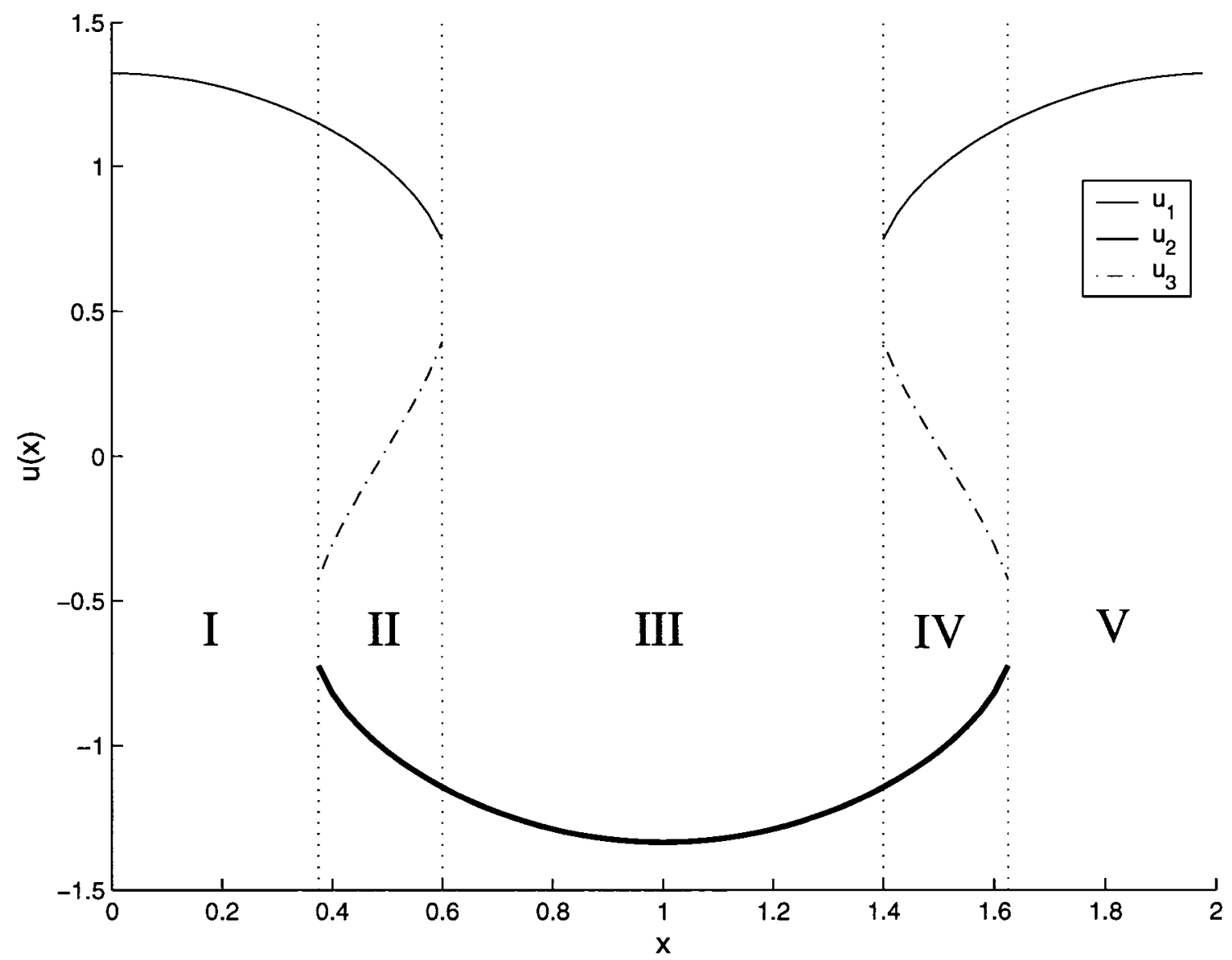

Figure 13: Partial real non-dispersive solutions. The various regions I-V are discussed in the text. 


$$
\gamma u_{x x}=\frac{\beta}{3} u^{3}+\Delta u-C .
$$

The phase plot of (21) is shown in Figure 14 for $\beta=3$, and in the two instances that $C=0$ and $C=1$. The non-dispersive solutions (for fixed $x$ ) in (14) are exactly the fixed points of the phase plot. We can then think of the forcing as causing a slow horizontal shift of the plane underneath the solutions; a solution beginning at one of the fixed points will thus remain at the 'fixed' point, but now slowly varies in response to the forcing.
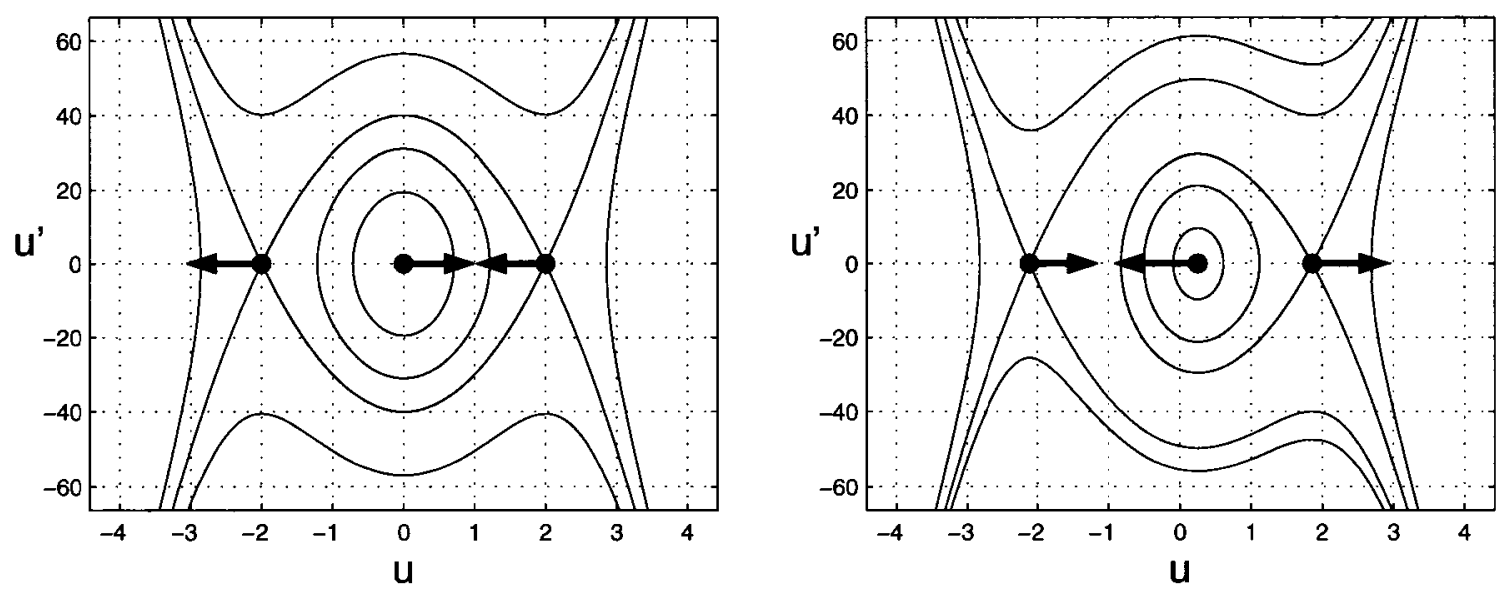

Figure 14: Phase plane of the undamped mKdV equation. The effect of the forcing is to provide an underlying shift in the phase plane.

But what about solutions in the resonant band? How do they behave in the phase plane? Although we have not yet studied these solutions in detail, we did manage to reveal in the previous section that they would involve transitions between the two flanking roots (or non-dispersive solutions). Can we thus expect these resonant solutions to transition between the two saddle points in the phase planes of Figure 14, perhaps closely following along the heteroclinic? We will explore this possibility soon enough. 
In Section 3.5, we will also take in account the small damping $\mu \sim \mathcal{O}(\gamma)$. Fortunately, this physically relevant parameter will only change the undamped solutions slightly, shifting the location of the boundary layer. The snapshot phase plots will also change, as the effect of damping breaks the symmetry and the closed orbits now become inward spirals, as in Figure 15

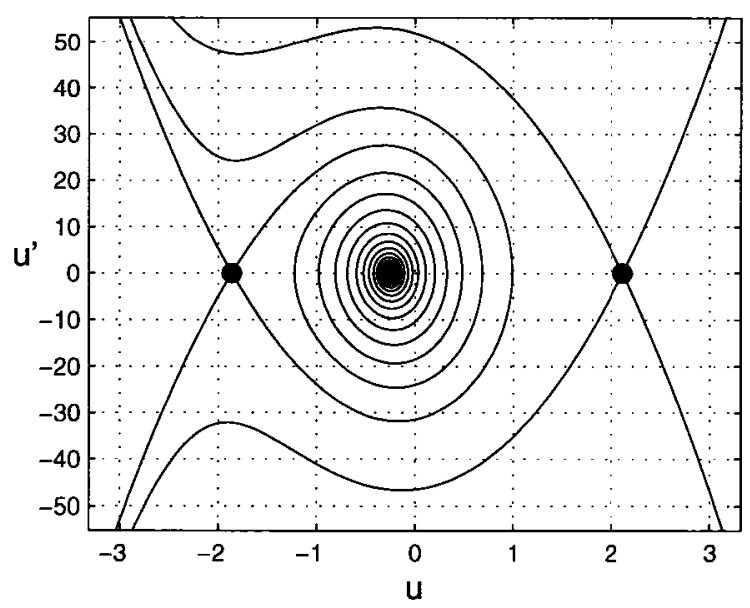

Figure 15: Phase Plane of the Damped Equation. Damping changes closed orbits to spirals.

\subsubsection{Energy Analysis and the Quartic Equation}

As with our examination of the cubic equation, equally revealing properties can be discovered by studying the integrated cubic, or energy equation of the undamped $\mathrm{mKdV}$ equation. To proceed with the analysis, we can multiply equation (13) by $u_{x}$ and integrate further, yielding

$$
\frac{6 \gamma}{\beta}\left(u_{x}\right)^{2}=u^{4}+\left(\frac{6 \Delta}{\beta}\right) u^{2}-\left(\frac{12 C}{\beta}\right) u+E \equiv H(u),
$$

once $\mu$ has been set to zero and the equation rearranged. 
The behaviour of the quartic polynomial $H(u)$ is of key importance to the solutions of the $\mathrm{mKdV}$ and an elementary study of the roots of the quartic polynomial can yield a wealth of qualitative details. Since for practical applications we are only interested in real solutions $u$ of equation (22), we will require that $\left(u_{x}\right)^{2} \geq 0$ and so we can expect solutions to vary monotonically (for the constant perturbed function) until $u_{x}$ vanishes at one of the roots of the quartic.

Thus for each value of the forcing, $C$ and for each choice of solution curve in the phase plane, $E$, we have a quartic polynomial with four (possibly complex or real repeated) roots. The particular solution $u(x)$ associated with these particular constants will be in one of the different possible regions with $H(u) \geq 0$.

There are several key observations to be made:

- First, it is quite simply seen that the non-dispersive solutions (15) are precisely the maxima and minima of the quartic polynomial $H(u)$.

- If $\bar{u}$ is a simple zero of $H(u)$, then an expansion of $H(\bar{u})$ reveals,

$$
\begin{aligned}
2 \gamma\left(u^{\prime}\right)^{2} & =H(\bar{u})+(u-\bar{u}) H^{\prime}(\bar{u})+\mathcal{O}\left((u-\bar{u})^{2}\right) \\
2 \gamma\left(u^{\prime}\right)^{2} & \sim(u-\bar{u}) H^{\prime}(\bar{u})
\end{aligned}
$$

as $u \rightarrow \bar{u}$. Now if we express $u(x)$ as a Taylor series about the point $x=\bar{x}$, where $u(\bar{x})=\bar{u}$, then

$$
u=\bar{u}+u^{\prime}(\bar{x})(x-\bar{x})+\frac{u^{\prime \prime}(\bar{x})}{2}(x-\bar{x})^{2}+\mathcal{O}\left((x-\bar{x})^{3}\right)
$$

and noting that $u^{\prime}(\bar{x})=0$ since $H(\bar{u})=0$ yields,

$$
u=\bar{u}+\frac{u^{\prime \prime}(\bar{x})}{2}(x-\bar{x})^{2}+\mathcal{O}\left((x-\bar{x})^{3}\right)
$$

And now differentiation of the energy equation (22) allows us to substitute, $u^{\prime \prime}(\bar{x})=H^{\prime}(\bar{u})$ into the previous equation,

$$
u \sim \bar{u}+\frac{1}{2} H^{\prime}(\bar{u})(u-\bar{u})^{2}
$$


where we have considered the limit that $x \rightarrow \bar{x}$. Thus, $u$ has a local minimum at $x=\bar{x}$ if $H^{\prime}(\bar{u})$ is strictly positive or a local maximum at $x=\bar{x}$ if $H^{\prime}(\bar{u})$ is strictly negative, respectively.

- If instead $\bar{u}$ is a double zero, then in the limit $u \rightarrow \bar{u}$, the approximation of equation (22) changes instead to,

$$
\left(u^{\prime}\right)^{2} \sim H^{\prime \prime}(\bar{u})(u-\bar{u})^{2}
$$

where we note $H^{\prime \prime}(\bar{u})>0$ and this can be integrated to yield,

$$
u \sim \bar{u}+\tilde{C} e^{ \pm x \sqrt{H^{\prime \prime}(\bar{u})}}
$$

Thus for solutions near a double root, we must have $u \rightarrow \bar{u}$ as $x \rightarrow+\infty$ or $u \rightarrow \bar{u}$ as $x \rightarrow-\infty$ (or both).

- This analysis of the quartic roots allows us to anticipate the types of rootarrangements we will require for our asymptotic analysis in the next section. There, we will be mainly interested in solutions $u(x)$ found between two simple roots of $H(u)$. For instance, consider Figure 16. Here, we have a simple zeros at a local max and a local min. Thus $u_{x}$ changes signs at these points and since the behaviour is algebraic (as opposed to the exponential behaviour with a double root), we would expect solutions to oscillate between the two roots with finite period.

- Also, let us examine the case when $u$ is located near a double root, $u=\overline{u_{1}}, \overline{u_{2}}$. Recall that the maxima and minima of the quartic are exactly the non-dispersive solutions (15). It should thus be clear that in the case of two double roots (Figure 17), the bounded solutions we expect to occur are monotonically increasing or decreasing, and approaching the non-dispersive solutions $\overline{u_{1}}$ and $\overline{u_{2}}$ as $x$ tends to $+\infty$ or $-\infty$ (assuming the positive branch of the square root of $\left(u_{x}\right)^{2}$ is chosen). 


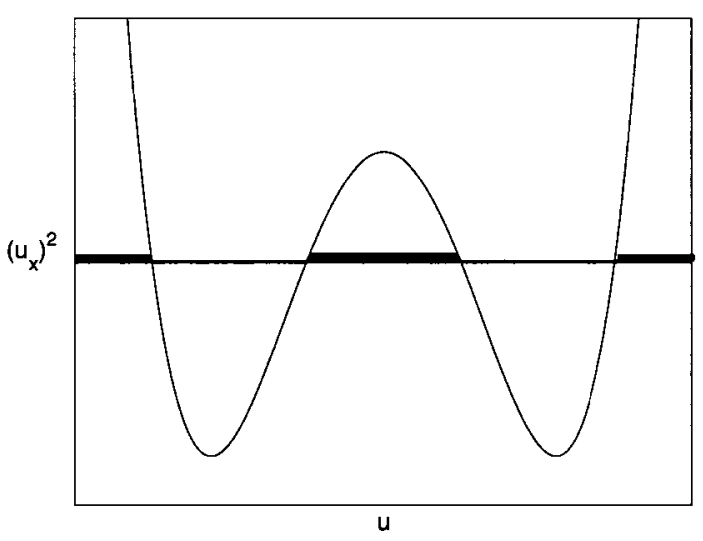

Figure 16: Quartic $H(u)$ has four distinct roots. Note if $u$ is between the two central roots, it will oscillate back and forth.

If instead $u$ is taken to be between a double and a single root (Figure 18), we would expect a $\max / \mathrm{min}$ to occur at the single root where $u_{x}$ changes signs, and thus it approaches the double root (non-dispersive solution) as $x \rightarrow \pm \infty$, forming a solitary wave.

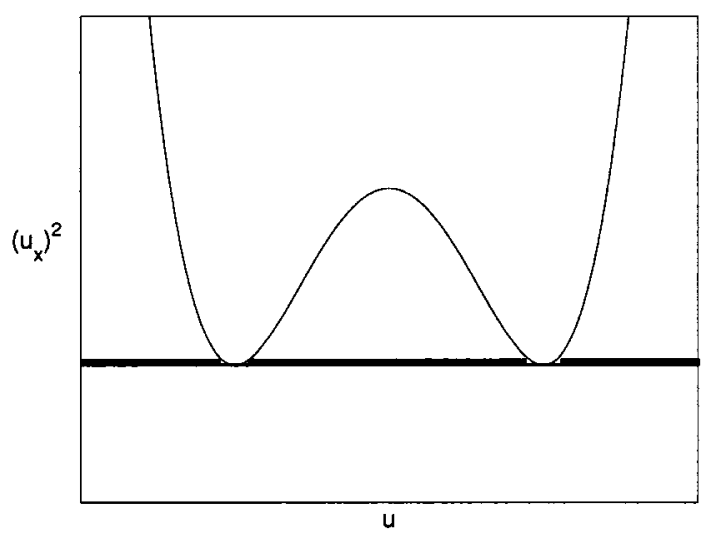

Figure 17: Two double roots. If $u$ is between the two roots, the solution will approach distinct values as $x \rightarrow \pm \infty$

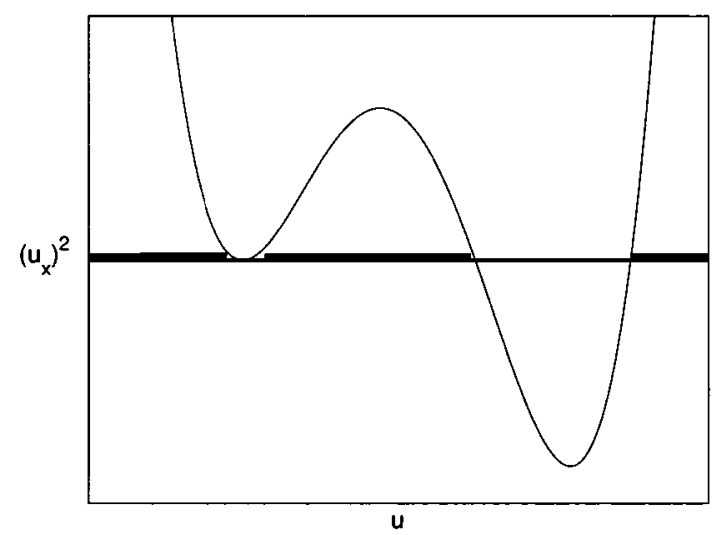

Figure 18: A Double and a single root. If $u$ is between the two roots, the solution will resemble a solitary wave.

- Although there are other (unbounded) solutions corresponding to cases where the solution lies outside the roots, these are not of particular interest. We 
will later see that the oscillatory solutions (between two single roots) and the double root solutions play a pivotal role in the derivation of the asymptotic solutions. In essence, the boundary layer will consist of oscillatory solutions with a slowly varying period (as the quartic shifts in response to the forcing), and the matching with the outer layers (non-dispersive solutions) will be accomplished by having the solution approach a double root.

In summary: The primary objective of these past few sections was to introduce the reader to the types of solutions that may arise from studying the forced $\mathrm{mKdV}$. We began by deriving the non-dispersive solutions $u_{1,2,3}$ in (15) which formed the fixed points in the subsequent phase plane analysis. By studying these equations in greater detail, we realized that they could not be valid solutions within a range of $\Delta$, the resonant band. We then explained how the phase plane of the forced mKdV could be interpreted as possessing an underlying shift due to the forcing, with the solutions adjusting appropriately. Finally, we studied the equivalent energy equation (22) and related the quartic roots with the types of solutions we might expect. Understanding these elementary facts will help immensely with the asymptotic analysis in the next section.

\subsection{Asymptotic Analysis of the Undamped Equation}

With a clear idea of our task at hand, we now look to derive the leading order asymptotic solutions of the undamped forced $\mathrm{mKdV}$, which is repeated here for convenience,

$$
\gamma u_{x x}=\frac{\beta}{3} u^{3}+\Delta u-(F(x)+c)
$$

Remember only for sufficiently large values of $|\Delta|$ can the zero-mean condition (12) be satisfied solely by the non-dispersive solutions $u_{1,2,3}$ indicated in (15). For the values of $\Delta$ lying within the resonant band, boundary layer solutions need to be derived. And from our qualitative analysis in the previous section, we can expect 
these dispersive solutions to be oscillatory in nature and occurring on a fast time scale.

Our asymptotic analysis will proceed with the following methodology (see also Figure 19):

1. Introduce a multiply-scaled function $u(x) \rightarrow u(x, X)$ where $X$ is the (fast) boundary-layer scaling and use a perturbative expansion of the function in orders of $\sqrt{\gamma}$.

2. Transform the differential equation and examine each separate order.

3. Solve for the leading order solution $u_{0}(x, X)$.

4. To avoid secular terms, impose a constant period $T$, and derive the periodicity condition involving the $\mathcal{O}(\sqrt{\gamma})$ terms. This provides an equation relating the energy $E$ to the slow variation $x$.

5. Match the dispersive solutions to the non-dispersive solutions.

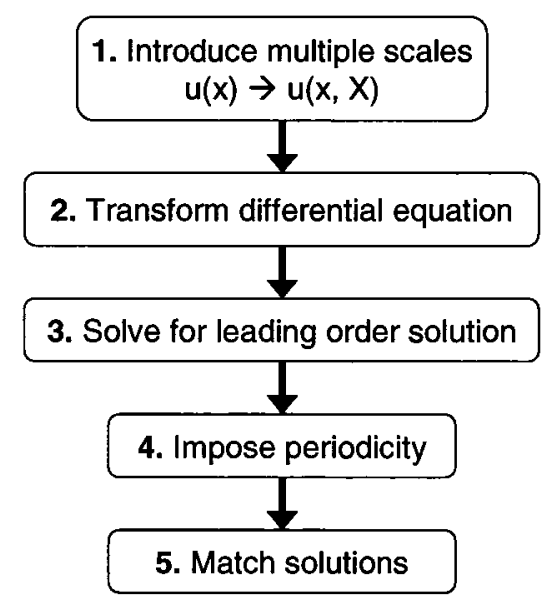

Figure 19: Our plan of attack. 


\subsubsection{Deriving the Dispersive Solutions}

To begin, we let,

$$
u(x) \rightarrow u(x, X),
$$

where we now allow $u$ to be a function of two independent scalings: a slow variable $x$ and a fast variable $X$, where

$$
X=\frac{\omega_{1}(x)}{\sqrt{\gamma}}+\omega_{2}(x)+\sqrt{\gamma} \omega_{3}(x)+\ldots
$$

This more general expansion is required, instead of the more typical linear scaling occurring in usual problems in multiple scales. This is in order to account for cases where the boundary layer may not be quite as small and the variation in the forcing needs be taken into account. Our need for the functional dependence $\omega_{1}(x)$ will emerge a little bit later.

By the chain rule, the ordinary differential operator now becomes,

$$
\begin{aligned}
\frac{d}{d x} & \rightarrow \frac{\partial}{\partial x}+\frac{\partial X}{\partial x} \frac{\partial}{\partial X} \\
& =\frac{\partial}{\partial x}+\left(\frac{\omega_{1}^{\prime}(x)}{\sqrt{\gamma}}+\omega_{2}^{\prime}(x)+\sqrt{\gamma} \omega_{3}^{\prime}(x)+\ldots\right) \frac{\partial}{\partial X}
\end{aligned}
$$

And the second order operator becomes,

$$
\begin{aligned}
\frac{d^{2}}{d x^{2}}= & {\left[\frac{\partial}{\partial x}+\left(\frac{\omega_{1}^{\prime}(x)}{\sqrt{\gamma}}+\omega_{2}^{\prime}(x)+\ldots\right) \frac{\partial}{\partial X}\right]\left[\frac{\partial}{\partial x}+\left(\frac{\omega_{1}^{\prime}(x)}{\sqrt{\gamma}}+\omega_{2}^{\prime}(x)+\ldots\right) \frac{\partial}{\partial X}\right] } \\
= & \frac{\partial^{2}}{\partial x^{2}}+2\left(\frac{\omega_{1}^{\prime}(x)}{\sqrt{\gamma}}+\omega_{2}^{\prime}(x)+\ldots\right) \frac{\partial^{2}}{\partial X \partial x} \\
& +\left(\frac{\omega_{1}^{\prime}(x)}{\sqrt{\gamma}}+\omega_{2}^{\prime}(x)+\ldots\right)^{2} \frac{\partial^{2}}{\partial X^{2}}+\left(\frac{\omega_{1}^{\prime \prime}(x)}{\sqrt{\gamma}}+\omega_{2}^{\prime \prime}(x)+\ldots\right) \frac{\partial}{\partial X} \\
= & \frac{1}{\gamma}\left(\omega_{1}^{\prime 2} \frac{\partial^{2}}{\partial X^{2}}\right)+\frac{1}{\sqrt{\gamma}}\left(2 \omega_{1}^{\prime} \frac{\partial^{2}}{\partial X \partial x}+2 \omega_{1}^{\prime} \omega_{2}^{\prime} \frac{\partial^{2}}{\partial X^{2}}+\omega_{1}^{\prime \prime} \frac{\partial}{\partial X}\right)+\mathcal{O}(1)
\end{aligned}
$$

We now introduce the perturbation expansion, 


$$
u(x, X)=u_{0}(x, X)+\sqrt{\gamma} u_{1}(x, X)+\gamma u_{2}(x, X)+\ldots
$$

The result of substituting the transformed differential operator, as well as the stretched coordinates, and perturbation expansion into the mKdV equation (23) yields the first two orders,

$$
\begin{aligned}
\mathcal{O}(1): & \left(\omega_{1}^{\prime}\right)^{2} u_{0 X X}=\frac{\beta}{3} u_{0}^{3}+\Delta u_{0}-(F(x)+c) \\
\mathcal{O}(\sqrt{\gamma}): & \left(\omega_{1}^{\prime}\right)^{2} u_{1 X X}-\beta u_{0}^{2} u_{1}-\Delta u_{1}=-2 \omega_{1}^{\prime} \omega_{2}^{\prime} u_{0 X X}-2 \omega_{1}^{\prime} u_{0 x X}-\omega_{1}^{\prime \prime} u_{0 X}
\end{aligned}
$$

It is quite convenient at this point to introduce the linear operator $L$, defined by,

$$
L \equiv\left(\omega_{1}^{\prime}\right)^{2} \frac{\partial}{\partial X^{2}}-\beta u_{0}^{2}-\Delta
$$

and it is easily seen that the higher order equations of (23) are given by,

$$
\mathcal{O}\left(\gamma^{k / 2}\right): L\left(u_{k}\right)=R_{k}, \quad k=1,2,3, \ldots
$$

where for example, $R_{1}$ is the right hand side of (30). We will now look to solve the $\mathcal{O}(1)$ equation. As in Section 3.3, we integrate once to reveal the energy equation,

$$
\left(\frac{6 \omega_{1}^{\prime 2}}{\beta}\right) u_{0 X}^{2}=u_{0}^{4}+\left(\frac{6 \Delta}{\beta}\right) u_{0}^{2}-\left(\frac{12 C}{\beta}\right) u_{0}+E
$$

where $C=C(x)$ and $E=E(x)$. Now by integrating the equation, we can obtain the solutions by way of an inverse integral,

$$
\int^{u_{0}(x)} \frac{d u_{0}}{ \pm \sqrt{u_{0}^{4}+\left(\frac{6 \Delta}{\beta}\right) u_{0}^{2}-\left(\frac{12 C}{\beta}\right) u_{0}+E}}=\int^{X} \frac{\sqrt{\beta}}{\sqrt{6} \omega_{1}^{\prime}} d X=\frac{\sqrt{\beta}}{\sqrt{6} \omega_{1}^{\prime}}(X+\delta(x))
$$

Notice here that we have used the constant of integration $\delta(x)$ to represent both the phase shift due to the definite integral (in X), and also from the constant arising from the multiply parametered $u(x, X)$ function. The lower limit of integration is not quite arbitrary, as we will want to restrict the types of solutions that arise in 
conjunction with our boundary layer phenomenon. It is also well known that the integral of $1 / \sqrt{P}$, where $P$ is a polynomial of degree 3 or 4 can be represented in terms of elliptic functions of the first and second kind (see [1] and [9]).

While no harm is done if we were to leave our solutions as the inverse integral in (33), explicitly stating the corresponding elliptic functions can ease the numerical analysis, and more importantly, provides an intuitive interpretation of the solutions, making use of common properties of the elliptic and circular functions. To do this, we will first begin by assuming,

$$
\begin{gathered}
u^{4}+\left(\frac{6 \Delta}{\beta}\right) u^{2}-\left(\frac{12 C}{\beta}\right) u+E=(u-a)(u-b)(u-c)(u-d) \\
d \leq c \leq u \leq b \leq a
\end{gathered}
$$

and that the roots $a, b, c, d$ are all real-valued. We can then integrate equation (32) from the $c$-root with the assumption that the solutions of interest oscillate between roots $c$ and $b$ (see Section 3.3).

$$
\int_{c}^{u} \frac{d u}{ \pm \sqrt{(u-a)(u-b)(u-c)(u-d)}}=\int_{c}^{X} \frac{\sqrt{\beta}}{\sqrt{6} \omega_{1}^{\prime}} d X=\frac{\sqrt{\beta}}{\sqrt{6} \omega_{1}^{\prime}}(X+\delta(x))
$$

Using [9] and [18], we can see that this inverse integral involving the square root of a quartic polynomial with four distinct roots can be expressed as,

$$
\int_{c}^{u} \frac{d u}{ \pm \sqrt{(u-a)(u-b)(u-c)(u-d)}}=\frac{2}{\sqrt{(a-c)(b-d)}} F(\theta, k)
$$

where $F(\theta, k)$ is the elliptic integral of the first kind (see [9]) and,

$$
\theta=\arcsin \left(\sqrt{\frac{(b-d)(u-c)}{(b-c)(u-d)}}\right), \quad k=\sqrt{\frac{(b-c)(a-d)}{(a-c)(b-d)}}
$$

And so if we use this result (35) in our expression for the inverse integral (34) and invert the result, we obtain our desired boundary layer solution ${ }^{1}$,

\footnotetext{
${ }^{1}$ For convenience of notation, we have chosen to use $m$, the modulus-squared value in our formula instead of the modulus $k$. They are simply related by $m=k^{2}$.
} 


$$
\begin{gathered}
\text { Jacobi Elliptic Dispersive Solutions (mKdV) } \\
u_{0}(x, X)=\frac{-(b-d) c+\operatorname{sn}^{2}(B(x-\delta), m)(b-c) d}{-(b-d)+\operatorname{sn}^{2}(B(x-\delta), m)(b-c)} \\
B=\frac{\sqrt{\beta(a-c)(b-d)}}{\sqrt{24} \omega_{1}^{\prime}}, \quad m=\frac{(b-c)(a-d)}{(a-c)(b-d)}
\end{gathered}
$$

The analytical solution of the four roots of the quartic equation can of course be solved for, but we omit the solution here, instead directing the readers to Appendix $\mathrm{B}$, where the details can be found.

\subsubsection{Avoiding Secularity and the Solvability Condition}

So by now, we have managed to derive the leading order boundary layer solution of interest. However, on closer inspection there are still several relations to establish. In particular, the $\omega_{1}$ of our stretched coordinate must be determined, along with the energy, $E$ allowing us to calculate the quartic roots $a, b, c$, and $d$ involved in the solution. These parameters will be determined by matching our analytic approximations.

Before turning to the $\mathcal{O}(\sqrt{\gamma})$ terms, let us first examine the period of the dispersive solutions (37),

$$
T=\frac{2 K(m)}{B}=\frac{4 \sqrt{6} K(m)}{\sqrt{\beta(a-c)(b-d)}} \omega_{1}^{\prime}
$$

Here we used our previously derived expression for the modulus (37) and the fact the Jacobi $\operatorname{sn}^{2}$ function has period $2 K(m)$ (see [1]). Luke [26] showed that to avoid secular growth, the period of the solution $T$ must be constant. To see this, we can express the solution as $u_{0}(X+n T(x), x)$ and differentiate with respect to $x$,

$$
\begin{aligned}
\frac{\partial}{\partial x} u_{0}(X+n T(x), x) & =\frac{\partial}{\partial x}(X+n T(x)) u_{0 X}+u_{0 x} \\
& =\left(\frac{\partial X}{\partial x}+n \frac{d T}{d x}\right) u_{0 X}+u_{0 x} \\
& =n\left(\frac{d T}{d x}\right) u_{0 X}+\frac{\partial X}{\partial x} u_{0 X}+u_{0 x}
\end{aligned}
$$


and it is then clear that unbounded growth occurs unless $\frac{d T}{d X}=0$. The period is thus constant and we are free to choose any constant. Conveniently, we will let $T=\frac{4 \sqrt{6}}{\sqrt{\beta}}$ and this allows us to express,

$$
\omega_{1}^{\prime}(x)=\frac{\sqrt{(a-c)(b-d)}}{K(m)},
$$

establishing the connection between the stretched coordinate parameter $\omega_{1}(x)$ and the modulus $m(x)$. The relation between the modulus and the variation in $x$ can now be derived using the solvability condition. Recall that using our notation for the linear operator $L$ in $(31)$, the $\mathcal{O}(\sqrt{\gamma})$ equation in our perturbative hierarchy is,

$$
L\left(u_{1}\right)=R_{1}
$$

where $R_{1}$ is simply the expression detailed in (30). It is not difficult to see that if we take the derivative of (29) with respect to $X$, we have,

$$
\begin{aligned}
0 & =\frac{\partial}{\partial X}\left(\left(\omega_{1}^{\prime}\right)^{2} u_{0 X X}-\frac{\beta}{3} u_{0}^{3}-\Delta u_{0}+(F(x)+c)\right) \\
& =\left(\omega_{1}^{\prime}\right)^{2} u_{0 X X X}-\beta u_{0}^{2} u_{0 X}-\Delta u_{0 X}
\end{aligned}
$$

which is nothing more than $L\left(u_{0 X}\right)$ and we have found that $u_{0 X}$ is a homogeneous solution of the higher ordered equation (40). But now in order to assure periodicity at this order, the right hand side of (30) must be orthogonal to all periodic homogeneous solutions of (40). This is known as the Fredholm Alternative and provides the solvability condition,

$$
\int_{0}^{T} u_{0 X} R_{1} d X=0
$$

where again, $R_{1}$ is the right hand side of (30). Although we only need to deal with assuring periodicity at this particular order, it should be noted that the above solvability condition is required when examining the higher orders as well. It will be instructive if we derive this solvability condition explicitly and review the underlying 
reasons for the Fredholm Alternative. To this end, we multiply the left-hand side of (30) by $u_{0 X}$ and integrate over a complete period,

$$
\begin{aligned}
\int_{0}^{T} L\left(u_{1}\right) u_{0 X} d X= & \left(\omega_{1}^{\prime}\right)^{2} \int_{0}^{T} u_{1 X X} u_{0 X} d X-\int_{0}^{T} u_{1}\left(\beta u_{0}^{2} u_{0 X}+\Delta u_{0 X}\right) d X \\
= & \left.\left(\omega_{1}^{\prime}\right)^{2} \underbrace{\left(u_{0 X} u_{1 X}-u_{0 X X} u_{1}\right)}_{\text {zero by periodicity }}\right|_{0} ^{T} \\
& +\int_{0}^{T} u_{1}(\underbrace{u_{0 X X X}-\beta u_{0}^{2} u_{0 X}+\Delta u_{0 X}}_{\text {zero by (41) }}) d X \\
= & 0
\end{aligned}
$$

where we have applied the condition of periodicity and used the fact $u_{0 X}$ is a homogeneous solution of $L(u)$. So the left side is zero and we are again left with the condition,

$$
\int_{0}^{T} u_{0 X} R_{1} d X=0
$$

And thus this method of imposing periodicity through solving an adjoint equation is precisely the reason for the Fredholm Alternative. Let us go on and calculate the terms in the solvability condition above,

$$
\begin{aligned}
0 & =\int_{0}^{T} u_{0 X} R_{1} d X \\
& =\int_{0}^{T}\left(2 \omega_{1}^{\prime} \omega_{2}^{\prime} u_{0 X X}+2 \omega_{1}^{\prime} u_{0 x X}+\omega_{1}^{\prime \prime} u_{0 X}\right) u_{0 X} \partial X \\
& =2 \int_{0}^{T}(\underbrace{\omega_{1}^{\prime} \omega_{2}^{\prime} u_{0 X X} u_{0 X}}_{\text {(1) }}+\underbrace{\frac{d}{d x}\left(\omega_{1}^{\prime} u_{0 X}^{2}\right)}_{\text {(2) }}) \partial X
\end{aligned}
$$

(1) is an exact differential and will vanish due to periodicity, 


$$
\begin{aligned}
2 \int_{0}^{T} \omega_{1}^{\prime} \omega_{2}^{\prime} u_{0 X X} u_{0 X} \partial X & =\omega_{1}^{\prime} \omega_{2}^{\prime} \int_{0}^{T} \frac{d}{d X} u_{0 X}^{2} d X \\
& =\omega_{1}^{\prime} \omega_{2}^{\prime}\left(u_{0 X}^{2}(T)-u_{0 X}(0)\right) \\
& =0
\end{aligned}
$$

And thus the solvability condition becomes (2) only,

$$
\frac{d}{d x} \omega_{1}^{\prime} \int_{0}^{T} u_{0 X}^{2} d X
$$

If we make the substitution $Q=\int_{0}^{T} \omega_{1}^{\prime} u_{0 X}^{2} d x$, we arrive at the canonical form of the solvability condition,

$$
\frac{d Q}{d x}=0
$$

This expression is also commonly known as the adiabatic invariant (see for example, [41]) and thus by integration,

$$
\begin{aligned}
& \text { Solvability Condition (Undamped mKdV) } \\
& \qquad \omega_{1}^{\prime} \int_{0}^{T} u_{0 X}^{2} d X=\kappa
\end{aligned}
$$

where $\kappa$ is a constant. This crucial equation (46) is the final relation that allows us to solve for the modulus $m$, as a function of $x$. The particular value of $\kappa$ will be dependent on the matching criteria and will be discussed in Section 3.6.

The solvability condition provides the final relationship between the modulus (or energy) values and the $x$ variation. There are, however, still several parameters which we have not yet examined: $\kappa$ from the above solvability condition, the phase shift $\delta(x)$ corresponding to our stretched coordinate, and $c$ the constant of integration from the forcing. For the moment, we leave these issues until Section 3.6, when we discuss the matching criteria.

In the next section, we study the $\mathrm{mKdV}$ with damping $\mu \neq 0$, but in general, the bulk of our work has already been done. As we shall see, the only effect of the 
damping, due to a slight change of our solvability condition in equation (46), is to change the location of the boundary layer .

\subsection{Asymptotic Analysis of the Damped Equation}

In the following section, we repeat the asymptotic analysis of the previous section, but now allow a non-zero damping $\mu \neq 0$ in our $\mathrm{mKdV}$ equation,

$$
\gamma u_{x x}+\mu u_{x}=\frac{\beta}{3} u^{3}+\Delta u-(F(x)+c)
$$

where, motivated by the physical scaling of the problem, we assume that $\mu \sim \mathcal{O}(\sqrt{\gamma})$.

As we mentioned during our qualitative discussion in Section 3.3, the effect of the damping will be to break the inherit symmetry of the undamped case, turning the closed orbits around the centre fixed point into inward spirals, and causing dispersive solutions to leave the non-dispersive solutions at different points (See Figure 15).

Since the introduction of the damping does not change the leading order expression in our regular perturbation series $u=u_{0}+\sqrt{\gamma} u_{1}+\ldots$, the leading order equation remains the same and in particular, our non-dispersive solutions are given by (15),

$$
u_{1,2,3}=\frac{\rho^{1 / 3}}{2 \beta} e^{2 \pi i k}-\frac{2 \Delta}{\rho^{1 / 3}} e^{-2 \pi i k}, \quad k=0,1,2
$$

where the expressions for $\rho$ and $M$ are given by (15).

Determining the dispersive solutions is now an exercise in applying the same stretched coordinate (25) and perturbative expansion of (28) into (47) as in the undamped case. Notice that the highest order term in $\mu u_{X}$ using the transformed differential operator (26) is only of order $\mathcal{O}\left(\frac{\mu}{\sqrt{\gamma}}\right) \sim \mathcal{O}(\sqrt{\gamma})$, so the leading order equation remains the same as (29). Consequently, the leading order dispersive solutions are given by (37),

$$
u_{0}(x, X)=\frac{-(b-d) c+\operatorname{sn}^{2}(B(x-\delta), m)(b-c) d}{-(b-d)+\operatorname{sn}^{2}(B(x-\delta), m)(b-c)}
$$


where the parameters are defined previously in (37). The only significant change occurs with the $\mathcal{O}(\sqrt{\gamma})$ equation, which is now,

$$
\mathcal{O}(\sqrt{\gamma}): \omega_{1}^{2} u_{1 X X}-\beta u_{0} u_{1}-\Delta u_{1}=-2 \omega_{1}^{\prime} \omega_{2}^{\prime} u_{0 X X}-2 \omega_{1}^{\prime} u_{0 x X}-\omega_{1}^{\prime \prime} u_{0 X}-\frac{\mu}{\gamma} \omega_{1}^{\prime} u_{0 X}^{2}
$$

Notice that the left hand side is still the $L$ operator defined in (31) applied to $u_{1}$, and thus the homogeneous solution of $L(u)=0$ is $u_{0 X}$ as always. We can apply the same method in deriving the solvability condition as before. Multiplying the right hand side of (48) by $u_{0 X}$ and integrating over a period $T$ of the leading order solution yields the (now slightly modified) adiabatic invariant which must be zero:

$$
\frac{d}{d x} \omega_{1}^{\prime} \int_{0}^{T} u_{0 X}^{2} d X-\frac{\mu}{\gamma} \int_{0}^{T} \omega_{1}^{\prime} u_{0 X} d X=0,
$$

where we have used the same simplifications as in (44) by using the periodicity of $u_{0 X}$. Making the substitution,

$$
Q=\omega_{1}^{\prime} \int_{0}^{T} u_{0 X}^{2} d X
$$

reveals the canonical form of the adiabatic invariant,

$$
\frac{d Q}{d x}+\frac{\mu}{\gamma} Q=0
$$

with the solution,

$$
\begin{aligned}
& \text { Solvability Condition (Damped mKdV) } \\
& \qquad Q(x, m(x))=\omega_{1}^{\prime} \int_{0}^{T} u_{0 X}^{2} d X=\kappa e^{-\frac{\mu}{\gamma} x}
\end{aligned}
$$

Here, we have implicitly provided relation between $Q$, and the $x$ and $m$ values. Note that setting $\mu=0$, we arrive at the same equation as in the undamped case (46) derived in the previous section. Equation (52) serves two purposes: (1) It provides a location for the layer and (2) It relates the modulus (and hence, the energy $E$ ) to the spatial variation $x$ provided that a value of $\kappa$ is given. In the next section, we will 
describe the final steps in determining these and the other parameters found in our analytic expressions.

But we have made significant progress already, so let us review our findings. In this section, we modified the asymptotic analysis of the undamped mKdV equation to account for a small damping $\mu \neq 0$. But qualitatively, the only change in our derived leading order solutions is a phase shift of the dispersive peaks and so there is an inherit asymmetry seen in the solutions. Quantitatively, this is seen by the change in the adiabatic invariant by way of an exponential term. These conditions allowed us to completely determine the variation of the modulus $m$ as a function of the spatial variation $x$.

\subsection{Matching of Solutions}

At this point, we have determined nearly all the necessary components to describe the leading order behaviour of the $\mathrm{mKdV}$. We have expressed our leading order solutions $u_{0}$ using both a slowly varying non-dispersive solution (15) and as a quickly oscillating dispersive solution. But these solutions involved several variables yet to be determined: $\kappa$, the constant of our solvability condition $(52) ; c$, the constant of integration from the forcing; $\delta(x)$, the phase shift; and $m(x)$, the modulus-squared or 'energy' of the problem. In the Kuzmak-Luke Method upon which we based our asymptotic analysis, these parameters were found in the context of an initial value problem (see [25] and [26]); our problem is similar, and the parameters will be determined by matching the inner and outer solutions across the boundary layer interface, and then applying the condition of zero mean. We now study each parameter in turn.

\subsubsection{Determining $\kappa$ by Enforcing a Matching}

Recall that during our asymptotic analysis, we used the inverse integral of a quartic equation (32) to derive the dispersive solutions,

$$
\left(\frac{6}{\beta} \omega_{1}^{\prime 2}\right) u_{0 X}^{2}=u_{0}^{4}+\left(\frac{6 \Delta}{\beta}\right) u_{0}^{2}-\left(\frac{12 C}{\beta}\right) u_{0}+E
$$


In fact, this quartic was first encountered during our qualitative introduction in Section 3.3. Our analysis assumed that the quartic could be factored,

$$
u_{0}^{4}+\left(\frac{6 \Delta}{\beta}\right) u_{0}^{2}-\left(\frac{12 C}{\beta}\right) u_{0}+E=\left(u_{0}-a\right)\left(u_{0}-b\right)\left(u_{0}-c\right)\left(u_{0}-d\right)
$$

into four ordered roots, $a \geq b \geq c \geq d$, and in particular, the inverse integral was evaluated from the initial root $u_{0}=c$ and integrated in the region between $c$ and $b$. This classification allowed us to express the dispersive solutions as a rational expression of Jacobi Elliptic functions.

In order for us to match these dispersive solutions to our non-dispersive solutions $u_{1,2,3}$, we will need to return once more to the quartic polynomial arrangements. Previously, we showed that the non-dispersive solutions were located at the maxima and minima of the quartic. Consequently, a matching between the dispersive solutions (oscillating between $b$ and $c$ ) and the non-dispersive solutions requires $u$ to approach the maxima/minima as the fast variable $X$ tends to $\pm \infty$ and we leave the layer.

But from our discussion of solutions near a double root of the quartic, this clearly implies that to force a successful match between dispersive and non-dispersive solutions, we must let $u$ approach a double root arrangement. More formally, let $x=\tilde{x_{1}}, \tilde{x 2}$ be the matching locations. At this value of $x$, we will need,

$$
\begin{aligned}
u_{0}^{4}+\left(\frac{6 \Delta}{\beta}\right) u_{0}^{2}-\left(\frac{12 C}{\beta}\right) u_{0}+E= & (u-c)^{2}(u-b)(u-a) \\
= & u^{4}+(-2 c-a-b) u^{3}+\left(c^{2}+2 a c+2 b c+a b\right) u^{2} \\
& +\left(-c^{2} a-c^{2} b-2 a b c\right) u+a b c^{2}
\end{aligned}
$$

And now equating coefficients, we have,

$$
\begin{gathered}
\frac{\beta}{3} c^{3}+\Delta c-C=0 \\
b=-c-\frac{\sqrt{-2 \beta\left(\beta c^{2}+3 \Delta\right)}}{\beta} \\
a=-b-2 c
\end{gathered}
$$


but examining the last term of (53), note we must now restrict,

$$
E=a b c^{2}
$$

Also note that implicit in equation (54) for $b$ was a sign choice in order to preserve our ordering $b \leq a$. More importantly, we can now recognize $c$ in (53) as exactly the three non-dispersive solutions, as we expected all along. That is,

$$
c=u_{1}, u_{2}, \text { or } u_{3}
$$

Now determining the value of $\kappa$ in the solvability condition (52) is easily done: Given the location of the matching $x=\tilde{x_{1}}, \tilde{x_{2}}$, we calculate the value of $\omega_{1}^{\prime}(x)$ and the four roots $a, b, c, d$ using the formulae (53)-(55), making sure to choose the appropriate non-dispersive solution for $c$ where the matching occurs. This completely determines our dispersive solution $u_{0}$ and now $\kappa$ is given by the solvability condition,

$$
\kappa=\omega_{1}^{\prime}(x) e^{\frac{\mu}{\gamma} x} \int_{0}^{T} u_{0 X}^{2} d X
$$

evaluated at the point of matching. And of course in the case of an undamped equation $(\mu=0)$, the exponential term disappears.

\subsubsection{Determining $\omega_{1}(x)$ and $m(x)$}

Recall that $\omega_{1}$ appears in the functional dependence of the fast coordinate $X$ on the slow spatial variation $x(25)$, while the modulus-squared $m(x)$ allows the elliptic functions in equation (37) to vary slowly in response to the forcing. Once a value of $\kappa$ has been determined however, both parameters are easily solved as functions of $x$ : the $\omega_{1}$ using equation (39), and the modulus-squared $m(x)$ using equation (52).

\subsubsection{Determining the Phase Shift $\delta(x)$}

The reader should recall that the phase shift was originally encountered as a constant of integration arising in our inverse integral (34), or equivalently, can be interpreted 
as arising in our definition of $X$, the scaled parameter. As before, let us assume that the transition between inner and outer layers occurs at $x=\tilde{x_{1}}$ and $x=\tilde{x_{2}}$.

If one is unfortunate enough to make an incorrect choice for $\delta(x)$, the boundary layer solutions beginning at $x=\tilde{x_{1}}$ will not match correctly at the other side, $x=\tilde{x_{2}}$. Note then, that for a successful match, we will also require an appropriate choice of $\delta(x)$ such that only an integer number of peaks exist within the dispersive layer.

In general, the modulation in the phase shift is not an easy problem to solve. It was extensively studied for a more general class of nonlinear differential equations by Bourland and Haberman in [5], [6], and [7], and can be formally solved for by examining the $\mathcal{O}(\gamma)$ terms and imposing periodic constraints, similar to what we have done in Section 3.4.2. The details however, are not particularly relevant to our work.

It will suffice it to say that for leading order approximations when the number of oscillations within the dispersive layer is small (in most practical circumstances), the phase shift $\delta(x)$ can be taken as constant. However, the algebraic complexity of our leading order solutions (37) necessitates numerically solving for the phase $\delta$, whether one chooses to solve the higher ordered solvability condition (as Bourland and Haberman), or perhaps more easily, as a a shooting-type algorithm from $x=\tilde{x_{1}}$ to $x=\tilde{x_{2}}$.

\subsubsection{Determining $c$ from the Mean Condition}

Finally, we turn to the last unknown parameter in our analytic approximations: the constant $c$, which occurs as a constant of integration in (10). Effectively, $c$ is a parameter that delineates the particular solutions that may arise among the family of possible solutions of the $\mathrm{mKdV}$ equation. However, as a result of the global mean condition (12), not all these solutions are acceptable. The final step in the analysis will thus be to solve for the value of $c$, which is determined implicitly by the mean condition,

$$
\int_{0}^{2 L} u(x, c) d x=0
$$


where we have constructed $u(x, c)$ using the non-dispersive and dispersive solutions. The above integral cannot be expressed analytically, so in practice $c$ must be determined numerically.

\subsubsection{Summarizing the Parameter Regime}

It will do us well to review our work in this section in matching the dispersive and non-dispersive solutions and determining the parameters involved in our analytic expressions. In many mathematical problems, the subtle details are often much more troublesome than the main bulk of the work, and indeed the reader will probably agree that matching and polishing the asymptotic solutions we derived in Sections 3.4 and 3.5 was not at all trivial. It will be convenient to summarize the key steps in determining each of the unknown parameters addressed in our work:

\begin{tabular}{|c|c|c|}
\hline Parameter & Found Using & Equation \\
\hline \hline$\kappa$ & Matching inner and outer solutions using a double root & $(57)$ \\
\hline$m(x)$ & Solvability condition & $(52)$ \\
\hline$\omega_{1}(x)$ & Enforcing constant period & $(39)$ \\
\hline$\delta(x)$ & Shooting-type argument & N/A \\
\hline$c$ & Global mean condition & $(58)$ \\
\hline
\end{tabular}

\subsection{Comparison of Analytic Approximations and Numerical Solutions}

In this section, we will study the numerical solutions of the $\mathrm{mKdV}$, and comment on the effectiveness of our asymptotic approximations. Here, many of the characteristics and curiosities hinted at during our brief qualitative analysis in Section 3.3) will be cleared up and in the end, the reader should have a clear conception of what solutions to the forced $\mathrm{mKdV}$ are possible and perhaps more impressively, how these solutions can be predicted and analytically formed.

The particular mKdV equation under study is, 


$$
-\gamma u_{x x x}+\Delta u_{x}+\beta u^{2} u_{x}-\mu u_{x x}=f(x)
$$

where we have chosen the parameters as,

\begin{tabular}{|c|c|}
\hline Parameter & Value \\
\hline \hline$\gamma$ & 0.005 \\
\hline$\beta$ & 3 \\
\hline$\mu$ & 0.0015 \\
\hline$f(x)$ & $-\pi \sin (\pi x)$ \\
\hline
\end{tabular}

In the first section, we will numerically solve for (59) for various values of $\Delta$, and discuss the characteristics of the solutions in correspondence with the resonance curves. In the second section, we will match the asymptotic approximations to the typical solutions and discuss the general effectiveness of our methodology.

\subsubsection{Numerical Solutions}

To generate the solutions to equation (59) we use AUTO 97, a well-known software package for continuation and bifurcation problems in ordinary differential equations [15]. The main result of interest here is the resonance graph displayed in Figure 20. Each area of interest is labeled, and we will discuss each in turn.

\section{- Regions A and I}

One may begin studying the resonance curves by starting at points $\mathbf{A}$ and $\mathbf{I}$, and following the curve towards the outstretched fingers. As was mentioned previously, for sufficiently small and large values of $\Delta$, at least one of the nondispersive solutions $u_{1,2,3}$ exist and satisfies the zero mean condition. In region A, all three non-dispersive solutions are real, but only $u_{3}$ satisfies the mean condition. In contrast, region I contains only one real solution $u_{1}$, which also satisfies the mean condition, but here, $u_{2}$ and $u_{3}$ are both imaginary and real for all $x$. Figure 21 and 22 illustrates the slowly oscillating graph of these non-dispersive solutions. 


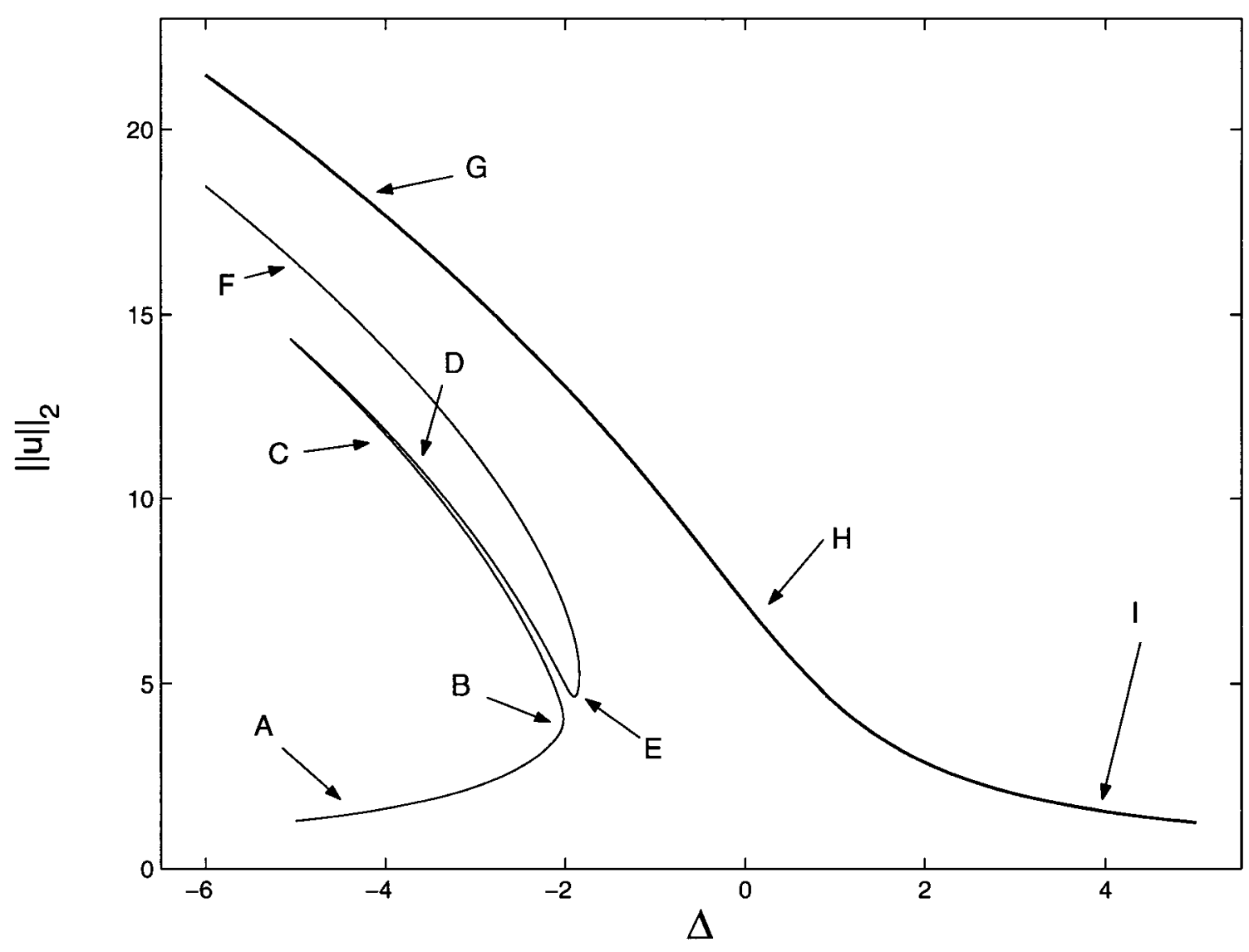

Figure 20: Resonance curves of the mKdV equation 59. The labeled regions are discussed in the text. We refer hereafter to the peaks of the figure as the 'fingers' of the resonance plot. 


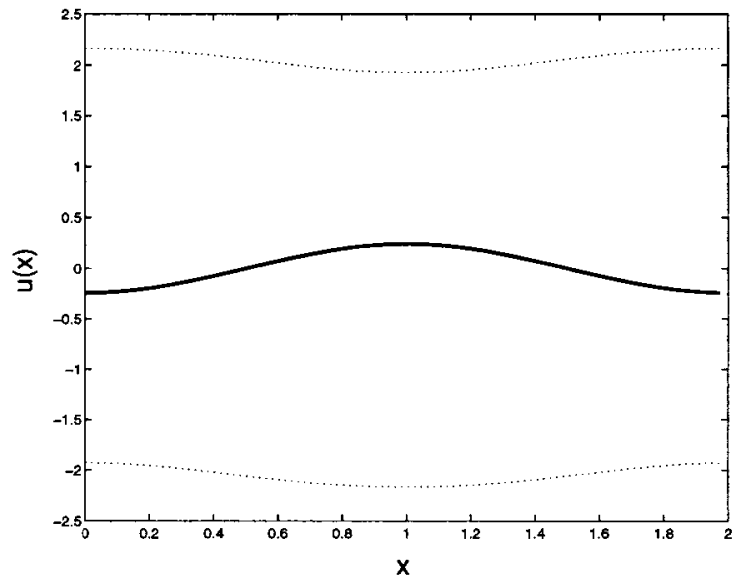

Figure 21: Region A

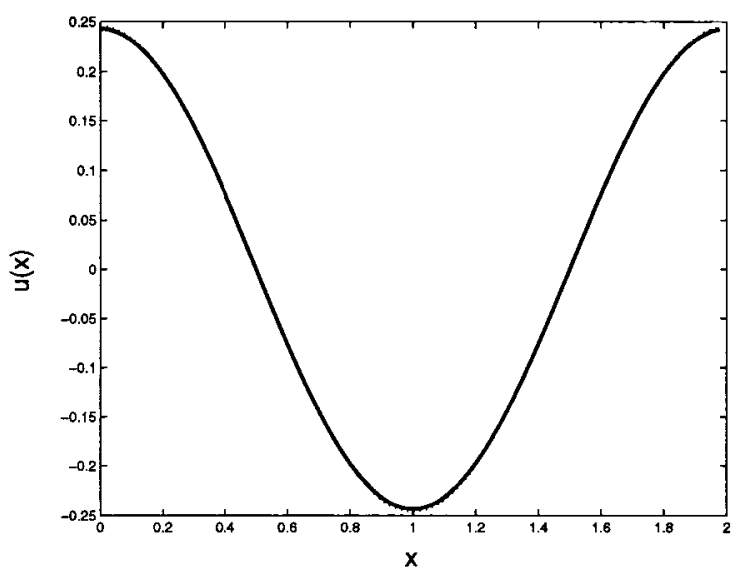

Figure 22: Region I

\section{- Regions B and E}

The 'bend' near regions $\mathbf{B}$ and $\mathbf{F}$ can be explained by our calculation of $\Delta_{M}^{*}$ in the previous section, which corresponds to the development of a cusp in all three non-dispersive solutions $u_{1,2,3}$. Using the approximation in (20) with $\beta=3$ and $\max _{x \in[0,2 L]}\left\{(F(x)+c)^{2}\right\} \approx 1$, we see that,

$$
\Delta_{M}^{*} \approx\left(\frac{-9}{4} 27^{3}\right)^{1 / 3} \approx 1.89
$$

which is indeed a good estimate of the location of the region. What is seen in region $\mathbf{B}$ is actually the formation of slightly faster oscillations about the $u_{3}$ solution which can be seen in Figure 23. The transition at region $\mathbf{E}$ and Figure 24 is more difficult to describe, and is more easily seen by comparing regions $\mathbf{D}$ and $\mathbf{F}$, with essentially the transition being in response to the (future) formation and desired avoidance of a cusp.

\section{- Regions H and G}

If the reader follows region I (Figure 22) backwards to $\mathbf{H}$ (Figure 25), the $u_{1}$ solution stretches in amplitude and more resembles the shape of a square pulse. The point of interest here is $\Delta^{*}=0$ calculated in equation (16) which corresponds to the point where $u_{1}$ develops a singularity. The key observation 


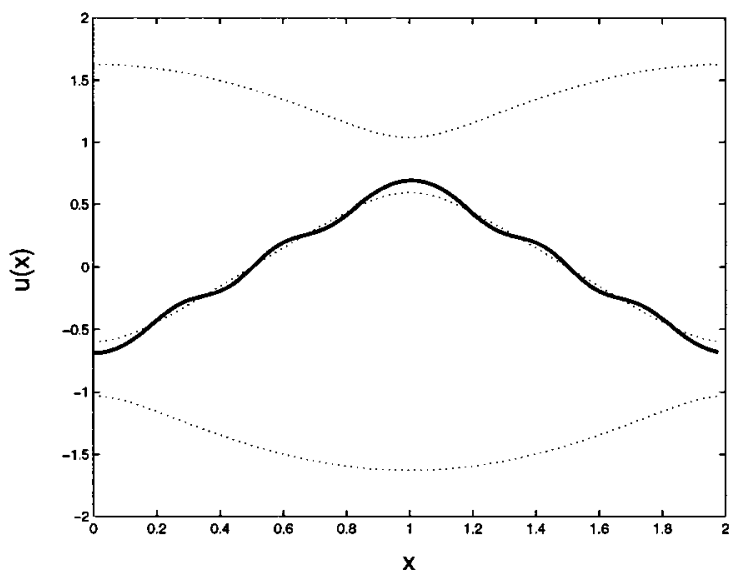

Figure 23: Region B

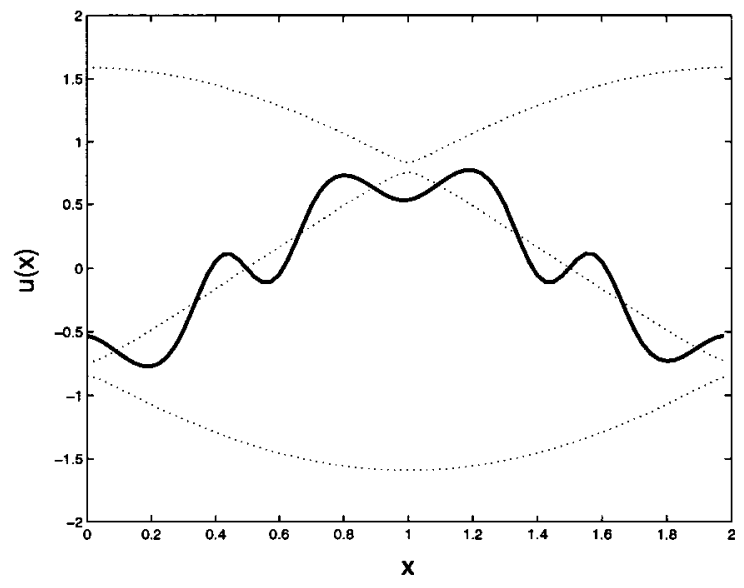

Figure 24: Region E

here is that the solutions, in response to this singularity have already began their transition into region $\mathbf{G}$ - where the top of the solution is attracted to the real remnants of $u_{1}$ and the bottom attracted to the real remnants of $u_{2}$. It is here where we see the first signs of a boundary layer which in essence, allows the solution to avoid complex regions of the non-dispersive solutions within the band $\Delta_{M}^{*}<\Delta<0$.

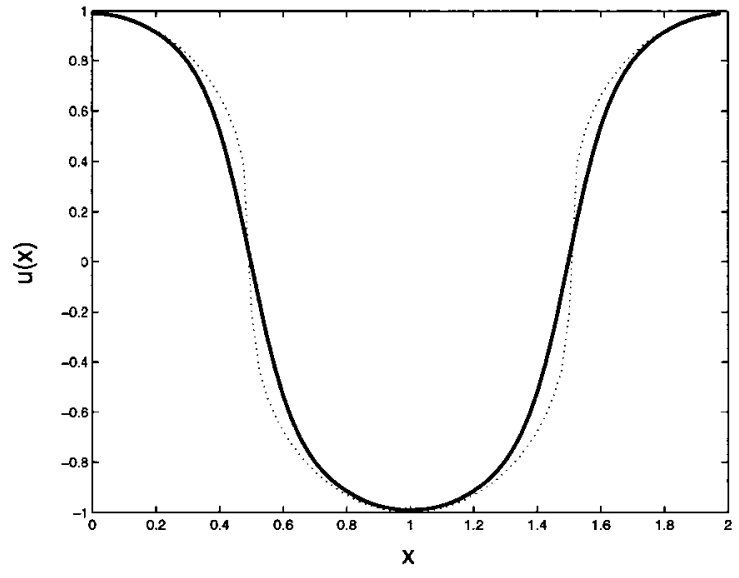

Figure 25: Region $\mathrm{H}$

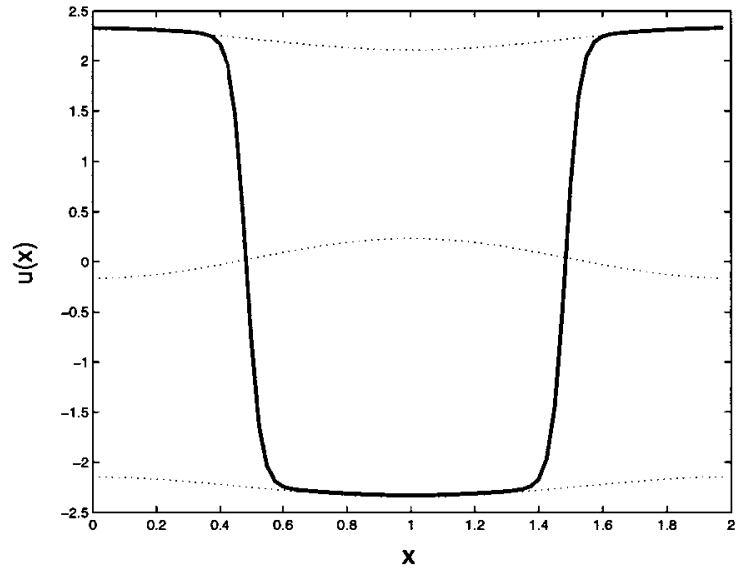

Figure 26: Region G

\section{- Regions C $\rightarrow \mathrm{D}$}

The main focus of these regions is the characteristic phase shift caused by the 
damping parameter $\mu$. Recall in Section 3.3, we showed that the introduction of damping resulted in an asymmetric shift in the boundary layer which was produced by an exponential term in the matching conditions. Figures 27 and 28 provide evidence of this reoccurent phase shift.

\section{- Regions $\mathbf{F} \rightarrow \mathrm{G}$}

Finally, the last few observations we make are found in Figures 28 and 29. Guiltily, we must admit that the figures are misleading and that unlike the solutions found transitioning from Regions $\mathbf{C}$ to $\mathbf{D}$, there is no easy way to see how Regions $\mathbf{F}$ and $\mathbf{G}$ are connected.

Guiltily, we admit that the resonance curves shown in Figure 20 are incomplete. Extending the domain of $\Delta$ to the left simply elongates the pre-existing fingers - even on the order of a hundred times the current size, the fingers do not seem to transition from left to right.

However, we shall see in Chapter 5 that indeed, the fingers do continuously transition from left to right and in fact, there are more fingers, not displayed in the resonance plots. This explains why the numbers of peaks within the dispersive layers differ from Figure 29 and 26: they are not even on the same finger! Numerically, the difficulty is the the enormous norms of the additional fingers. They are there; we simply can not see them. But the underlying reason why this occurs is not clear at the moment and we will defer the discussion until our examination of the eKdV in Chapter 5.

\subsubsection{The Effectiveness of the Derived Approximations}

Figure 31 provides an example of the high accuracy of approximating the solution using the leading order approximations. In particular, we have generated approximations to equation (59) for the two cases $\Delta=-5$ and $\Delta=-4.57$. Notice that the agreement is excellent and our approximations are not limited by the complex nature of these 'multiply-peaked' solutions. 


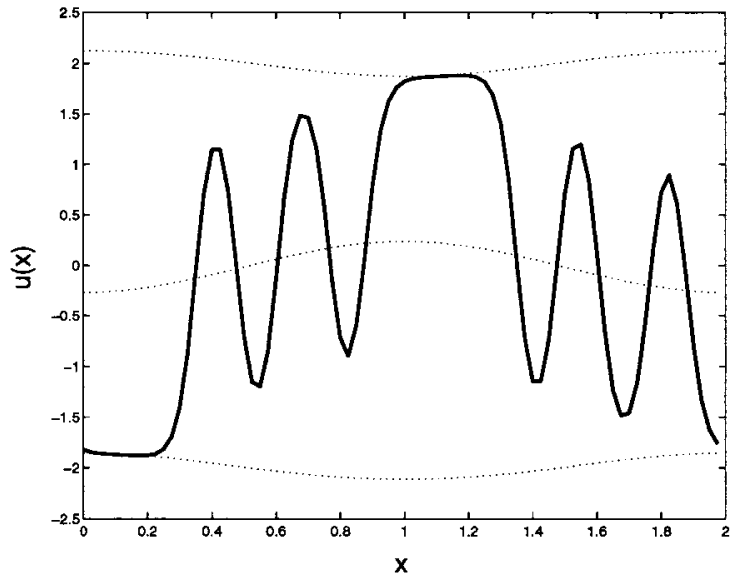

Figure 27: Region C

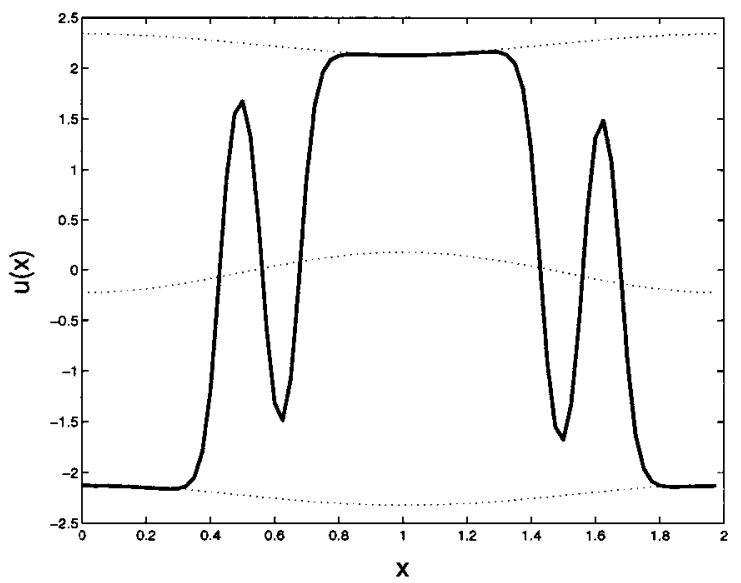

Figure 29: Region F

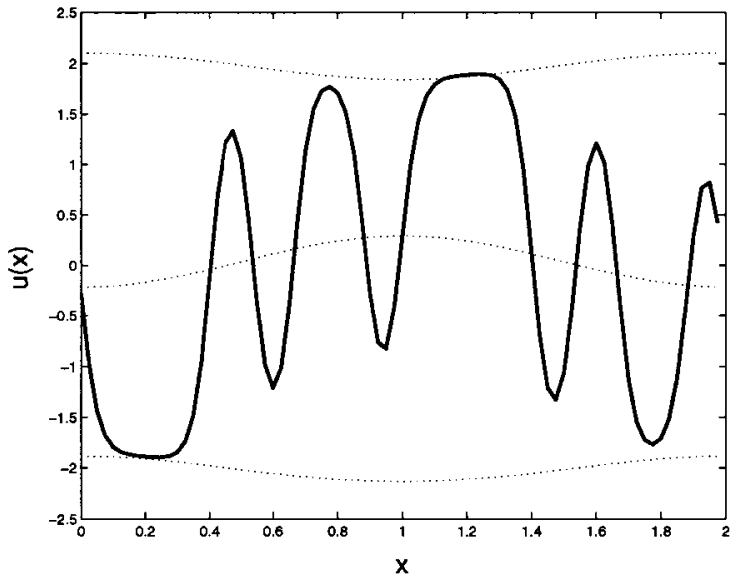

Figure 28: Region D

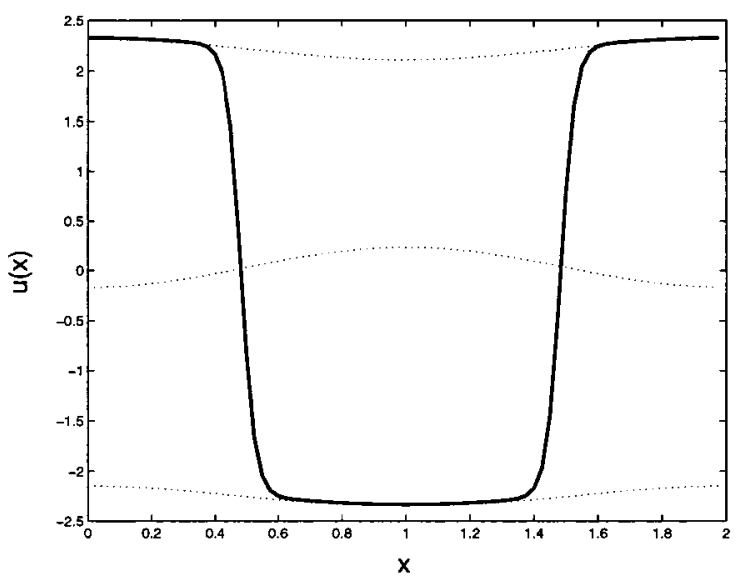

Figure 30: Region G 

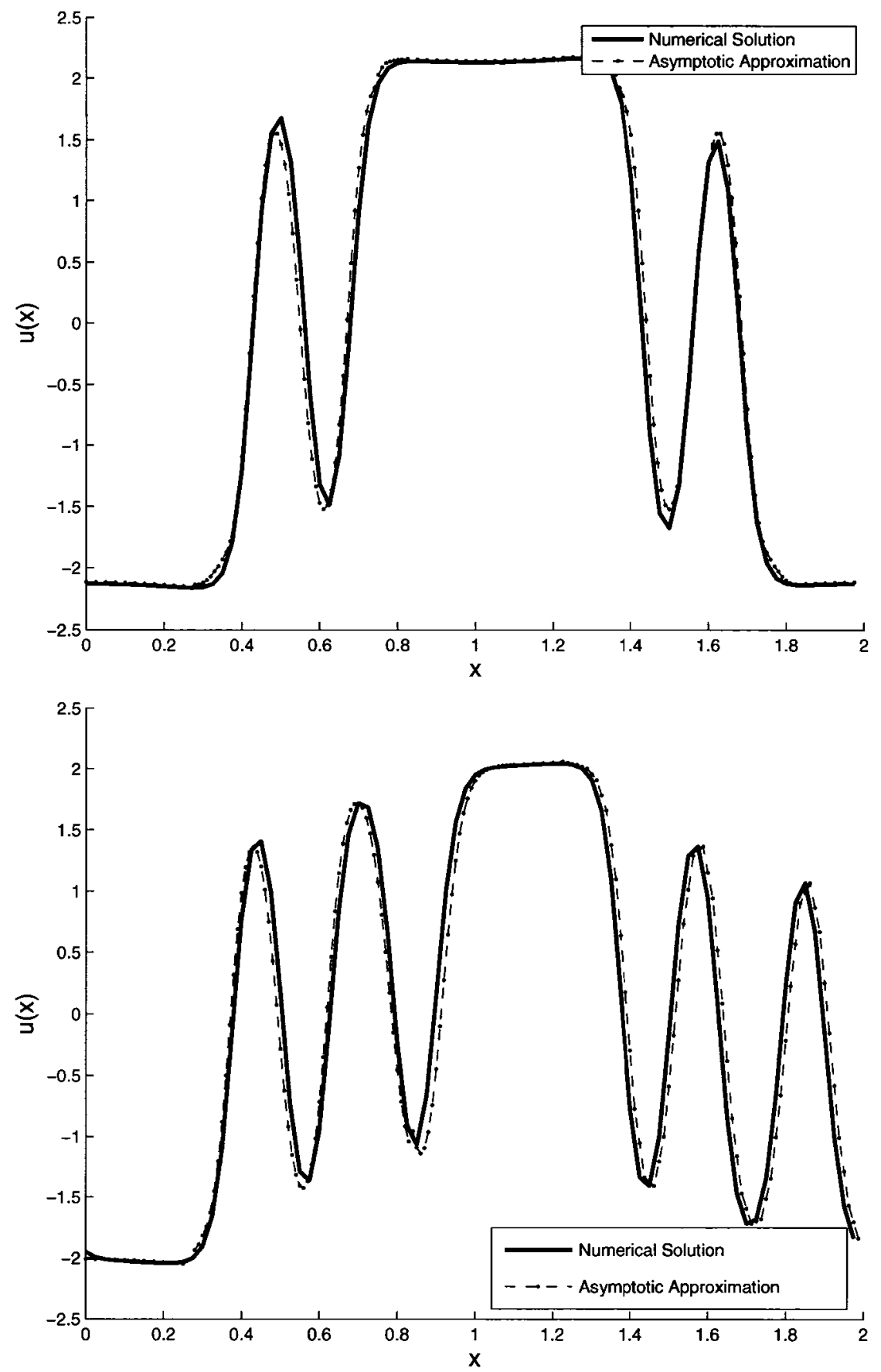

Figure 31: A comparison between the numerical solutions and leading order approximations of equation (59). The top figure is for $\Delta=-5$ while the bottom figure is for $\Delta=-4.57$ 


\subsection{A Novel Approximation to Singular Shocks}

In this section, we develop a novel but limited approximation scheme, originally conceived as a prelude to the more general elliptic solutions of Section 3.4. Often, it may be useful to sacrifice rigor and generality in exchange for quick analytic approximations which nonetheless illustrate the general nature of the solutions.

The idea is to use the elliptic solutions to an unperturbed $\mathrm{mKdV}$ equation and allow for minimal (constant) variation in the forcing at the edges, just enough to match the inner and outer solutions. This approximation is valid in the case of single shock solutions where the boundary layer is of size $\mathcal{O}(\gamma)$ akin the solutions of Figure 26 .

\subsubsection{Extensions using tanh}

Suppose we begin with the undamped mKdV in integrated form,

$$
\gamma u_{x x}=\frac{\beta}{3} u^{3}+\Delta u-[F(x)+c]
$$

In order to obtain the fast solution, we assume that the boundary layer is centred at $x=\delta$. However, unlike the analysis of Section 3.4, here we assume the forcing is zero within the layer. But for boundary layers with non-zero thickness, these primitive approximations are not sufficient to assure a match with the outer, non-dispersive solutions. To get around this, we will use a non-zero (but constant) forcing at the edges of the layer $x=\tilde{x_{1}}, \tilde{x_{2}}$.

\section{Re-scaling the Problem}

To begin with our usual method of multiple scales, we let,

$$
u(x) \rightarrow u(X, x)
$$

and,

$$
X=\frac{x-\delta}{\sqrt{\gamma}}
$$


We expand the forcing as,

$$
\begin{aligned}
F(x)+c & =[F(\delta)+c]+F^{\prime}(\delta)(x-\delta)+\frac{F^{\prime \prime}(\delta)}{2}(x-\delta)^{2}+\ldots \\
& =0+F^{\prime}(\delta)(x-\delta)+\frac{F^{\prime \prime}(\delta)}{2}(x-\delta)^{2}+\ldots
\end{aligned}
$$

where we have assumed that the forcing is zero at the centre of the layer, or $F(\delta)+c=0$.

Now expanding $u(X, x)=u_{0}(X, x)+\sqrt{\gamma} u_{1}(X, x)+\ldots$ and after the re-scaling of the derivatives, the first and second orders of equation (60) become,

$$
\begin{aligned}
\mathcal{O}(1): & u_{0 X X}=\frac{\beta}{3} u_{0}^{3}+\Delta u_{0} \\
\mathcal{O}(\sqrt{\gamma}): & u_{1 X X}-\beta u_{0}^{2} u_{1}-\Delta u_{1}=-F^{\prime}(\delta)-2 u_{0 X x} \equiv R_{1}
\end{aligned}
$$

where the $F^{\prime}(\delta)$ term arises due to our assumption that $(x-\delta) \sim \mathcal{O}(\sqrt{\gamma})$. This is the key condition that allows us to extend our leading order solutions at the boundary of the matching.

\section{Leading Order Approximation}

The leading order problem (63) an exercise in studying the unforced Duffing equation, and corresponds to the situation when the quartic of equation (22) is symmetric about the $u_{x}$-axis (Review our qualitative analysis in Section 3.3). By substitution, it can be seen that the solution of equation (63) can be represented by the Jacobi elliptic function,

$$
u_{0}=a(x) \operatorname{sn}(b(x) X, k(x))
$$

where,

$$
a(x)= \pm \frac{\sqrt{-6 \Delta} k}{\sqrt{\beta} \sqrt{1+k^{2}}}, \quad \text { and } \quad b^{2}=\frac{a^{2}}{2 k^{2}}
$$




\section{Avoiding Secularity and the Solvability Condition}

Next, we will show that after applying the secularity conditions, the the modulus $k$ must be equal to 1 .

As in Section 3.4, the secularity condition is,

$$
\int_{-\frac{T}{2}}^{\frac{T}{2}} R_{1} u_{0 X} d X=0
$$

where $R_{1}$ refers to the expression in (64) and not the one in Section 3.4.1. This becomes,

$$
\begin{aligned}
0 & =\int_{-\frac{T}{2}}^{\frac{T}{2}}\left(F^{\prime}(\delta)+2 u_{0 X x}\right) u_{0 X} d X \\
& =\int_{-\frac{T}{2}}^{\frac{T}{2}} F^{\prime}(\delta) u_{0 X} d X+\int_{-\frac{T}{2}}^{\frac{T}{2}}\left(u_{0 X}\right)^{2} d X \\
& =F^{\prime}(\delta)(u(T / 2)-u(T / 2))+\frac{\partial}{\partial x} \int_{-\frac{T}{2}}^{\frac{T}{2}}\left(u_{0 X}\right)^{2} d X
\end{aligned}
$$

But note that by properties of the Jacobi SN function, $\left.u\right|_{-\frac{T}{2}} ^{\frac{T}{2}}=0$ unless we choose to extend the period $T \rightarrow \infty$ and set the modulus $k=1$. This important restriction is necessary in order to apply a secularity condition involving the forcing. Thus, in this case the leading order approximation becomes,

$$
u_{0}=a(x) \tanh (b(x) X)
$$

And equation (67) yields,

$$
\begin{aligned}
0 & =2 F^{\prime}(\delta) a(x)+\frac{\partial}{\partial x} a^{2}(x) b^{2}(x) \int_{-\infty}^{\infty} \operatorname{sech}^{4}(b(x) X) d X \\
& =2 F^{\prime}(\delta) a(x)+\frac{d}{d x}\left(\frac{2 \sqrt{2}}{3} a^{3}(x)\right)
\end{aligned}
$$


The result is now a separable differential equation for the amplitude $a(x)$,

$$
\sqrt{2} \frac{d a}{d x} a+F^{\prime}(\delta)=0
$$

which yields solutions,

$$
a(x)= \pm \sqrt{-\sqrt{2} F^{\prime}(\delta)(x-\delta)+\Lambda}
$$

where $\Lambda$ is a constant of integration. By comparison with the unperturbed solutions of equation (66) with $k=1$, we can see that $\Lambda=\frac{3 \Delta}{\beta}$ after requiring $a(x)=\sqrt{\Delta}$ at the centre of the layer $x=\delta$. Choosing the sign of $a(x)$ corresponds to choosing whether to match on the left or right side of the boundary layer. This yields the final form of our approximation,

$$
\begin{gathered}
\text { Tanh Extensions (Single Shock mKdV Solutions) } \\
u(x)=u_{0}=a(x) \tanh \left(b(x) \frac{(x-\delta)}{\sqrt{\gamma}}\right) \\
a(x)= \pm \sqrt{-\sqrt{2} F^{\prime}(\delta)(x-\delta)+\frac{3 \Delta}{\beta}}, \quad b^{2}=\frac{a^{2}(x)}{2}
\end{gathered}
$$

\section{Summary and Comparison with Numerical Solutions}

To recap, we began by examining the centre of the boundary layer $(x=\delta)$. By treating the forcing as zero within this layer $(F(\delta)+c=0)$, we re-scale the problem and solve for an unforced Duffing equation. However, the key step of our approximation is imposing a minimal condition for a matching to occur with the assumption that $x-\delta \sim \mathcal{O}(\sqrt{\gamma})$ at the edges of the boundary layer. This assumption allows us to use the forcing in our secularity condition, yielding an equation to allow for the variation of the amplitude (and phase) of the tanh function. 
Figure 33 illustrates the effectiveness of this method in extending our tanh approximations to the case of single shock solutions with a sufficiently small boundary layer. The approximation is almost a perfect match on the left side of the solutions, with only a small deviation on the right, due to a phase shift from the damping discussed in Section 3.5 unaccounted for. Figure 32 provides a closeup of the crucial transition region between inner and outer layers. Notice that using a regular tanh solution valid in the unforced Duffing equation is not capable of providing a match, but by extending our approximations very slightly, we were able to extend the curve slightly, just enough to achieve a match.

\subsection{Summary}

- We derived the leading order non-dispersive solutions which are the solutions to a cubic equation. There is an interval for which the non-dispersive solutions can not alone satisfy the $\mathrm{mKdV}$ equation. This resonant band, $\Delta_{M}^{*} \leq \Delta \leq 0$ requires inclusion of dispersive effects.

- Using a method of multiple scales, leading order dispersive solutions were derived. These solutions incorporate the effect of slow variation in the forcing, and thus contains slowly varying parameters (such as the amplitude, wavenumber, and modulus).

- By matching slow (non-dispersive) and fast (dispersive) layers and providing a solvability condition, we were able to determine all the parameters involved in the leading order approximations.

- A new approximation method was proposed, consisting of extending simple tanh approximations and imposing a minimal condition for a matching. This approximation is valid in the limiting case of single 'shock' layers between nondispersive solutions.

- Agreement between numerical and approximate solutions is excellent. 


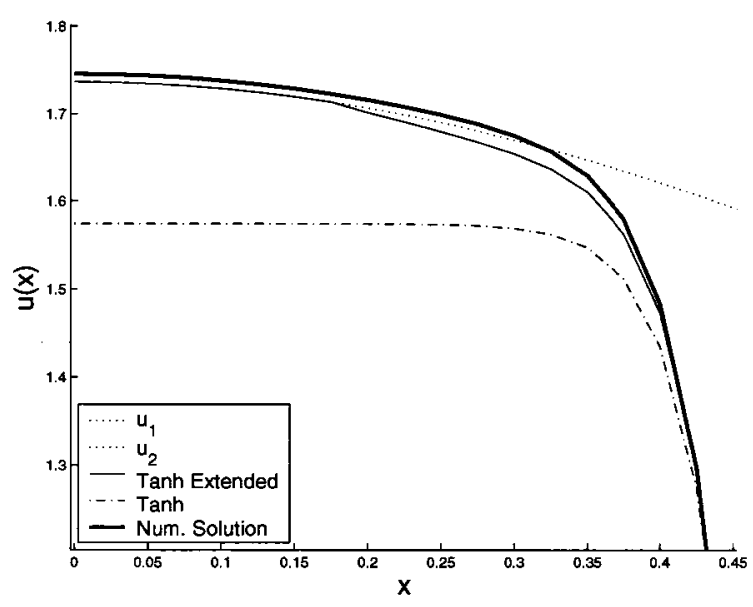

Figure 32: Closeup of the transition region using tanh extensions. Notice the obvious inability of the regular tanh in matching to the non-dispersive solutions

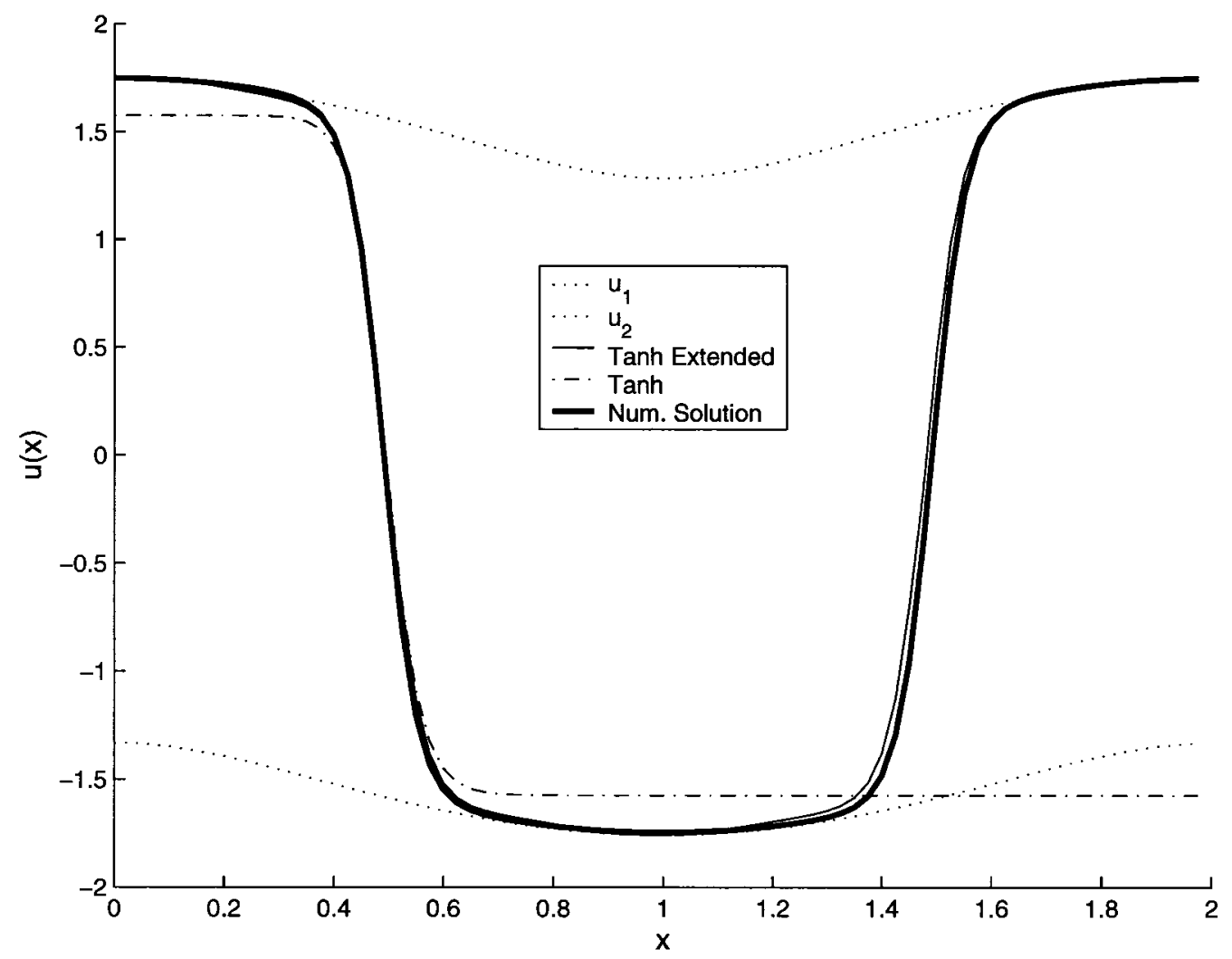

Figure 33: Approximating a singular shock solution using a tanh extension 


\section{Chapter 4}

\section{Comparing the $\mathrm{KdV}$ and $\mathrm{mKdV}$ Equations}

\subsection{Introduction}

In the first chapter, we introduced the reader to the illustrious $\mathrm{KdV}$ equation, and in particular spoke at length regarding the more typical forced $\mathrm{KdV}$ with quadratic nonlinearity and Burgers' type damping,

$$
u_{t}-\gamma u_{x x x}+\alpha u u_{x}+\Delta u_{x}-\mu u_{x x}=f(x) .
$$

In this chapter, we will summarize the previous work and results on the forced $\mathrm{KdV}$ by Amundsen, Cox, and Mortell [2] and in particular, seek to emphasize and compare particular differences between the $\mathrm{KdV}$ and the $\mathrm{mKdV}$. These subtleties may not have been particularly noteworthy when studying each equation separately, but taken together, they lead to a much greater understanding of the KdV-theory as a whole.

Our order of presentation will follow closely to that of the previous section: In Section 4.2 , we will open with the derivation of the non-dispersive solutions and discuss its existence and validity, which will be followed with a discussion of the $\mathrm{KdV}$ phase plane in Section 4.3. Next, we summarize the asymptotic analysis in Section 4.4 , and this will be compared and applied to numerical solutions discussed in Section 
4.5. The theme throughout this chapter is a comparison with the mKdV study in the previous chapter, and we will summarize our findings in Sections 4.6 and 4.7.

\subsection{Non-Dispersive Solutions}

Consider the steady state non-dimensionalized form of the forced KdV equation with Burgers' damping (fKdVB),

$$
-\underbrace{\gamma U_{x x x}}_{\text {dispersion }}+\underbrace{\Delta U_{x}}_{\text {detuning }}+\underbrace{\alpha U U_{x}}_{\text {quadratic nonlinearity }}-\underbrace{\mu U_{x x}}_{\text {damping }}=\underbrace{f(x)}_{\text {forcing }}
$$

defined over the domain $x \in[0,2 L]$. As before, $\gamma$ corresponds to the dispersion, $\alpha$ the degree of nonlinearity, $\Delta$ to the detuning from resonance, and $\mu$ is a measure of the damping. Both $\gamma$ and $\mu$ are assumed to be small and of the same order, the solutions and forcing are assumed to be smooth, and the forcing $2 L$ periodic. Additionally, the zero mean condition is also applied,

$$
\int_{0}^{2 L} U(x) d x=0
$$

which will serve to limit the types of solutions that may occur.

Note that we may assume $\alpha>0$ throughout our analysis since a simple change $U(x) \rightarrow-U(x)$ allows us to repeat the same arguments for the case that $\alpha<0$.

\subsubsection{A Transformation and the Non-Dispersive Solutions}

In contrast with the $\mathrm{mKdV}$, there is a particularly useful linear transformation here,

$$
U=u-\frac{\Delta}{\alpha}
$$

which serves to eliminate the $\Delta$ parameter in equation (73), yielding,

$$
-\gamma u_{x x x}+\alpha u u_{x}-\mu u_{x x}=f(x)
$$

but now the mean condition (74) changes to, 


$$
\int_{0}^{2 L} u(x) d x=\frac{2 L}{\alpha} \Delta .
$$

Integrating equation (75) once and rearranging yields,

$$
\gamma u_{x x}+\mu u_{x}=\frac{\alpha}{2} u^{2}-(F(x)+c)
$$

where we have defined $F(x)$ to be the anti-derivative of $f(x)$ and $c$ is a constant of integration. Like in the $\mathrm{mKdV}$, we can first proceed naively by substituting a regular perturbation expansion,

$$
u=u_{0}+\sqrt{\gamma} u_{1}+\gamma u_{2}+\ldots
$$

into equation (77). This yields the leading order non-dispersive solutions,

KdV Non-Dispersive Solutions

$$
u_{ \pm} \equiv \pm \sqrt{\frac{2}{\alpha}(F(x)+c)}
$$

\subsubsection{Existence of the Non-Dispersive Solutions and the Resonant Band}

Let us take a moment to analyze the existence and validity of these non-dispersive solutions. There is the obvious constraint,

$$
F(x)+c \geq 0
$$

for $x \in[0,2 L]$ which specifies a critical value of $c$,

$$
c^{*}=\max _{x \in[0,2 L]}(-F(x))
$$

where a cusp forms on the solution. The mean condition (76) then allows us to state this existence criterion using $\Delta$, 


$$
\Delta^{*}=\lim _{c \rightarrow c^{*}} \Delta=\lim _{c \rightarrow c^{*}} \frac{\alpha}{2 L} \int_{0}^{2 L} u_{+} d x .
$$

And thus for values of $\Delta$ such that,

$$
|\Delta|<\Delta^{*}
$$

the non-dispersive solutions alone cannot satisfy the global condition. This range of $\Delta$ is referred to as the resonant band.

\subsection{The Phase Plane}

The phase plane of the steady solutions of the undamped KdV equation,

$$
\gamma u_{x x}=\frac{\alpha}{2} u^{2}-C
$$

for fixed values of the forcing $C \equiv F(x)+c$, contains a centre $u_{+}$and a saddle $u_{-}$, enclosed by a homoclinic orbit. As we allow the forcing to vary, this shifts the plane and produces the slow oscillations found in our non-dispersive solutions (78). Figure 34 illustrates the phase plane for the $\mathrm{KdV}$ equation when the forcing $C \equiv F(x)+c=0$.

It is helpful to compare the KdV phase plot to that of the $\mathrm{mKdV}$ found in Figure 14. Note the additional saddle in the $\mathrm{mKdV}$ positioned to the left of the centre fixed point which provides a symmetry to the problem not found in the KdV. Also, a homoclinic serves as the separatrix in the $\mathrm{KdV}$ when there is no perturbation, but in the $\mathrm{mKdV}$, it is instead a heteroclinic that separates the bounded and unbounded solutions.

With these details in mind, we are now ready to proceed to the next section for the asymptotic analysis. We will return to the details of the phase plots in Section 4.6 when we compare the numerical and analytical solutions of the KdV and $\mathrm{mKdV}$ in full detail. 


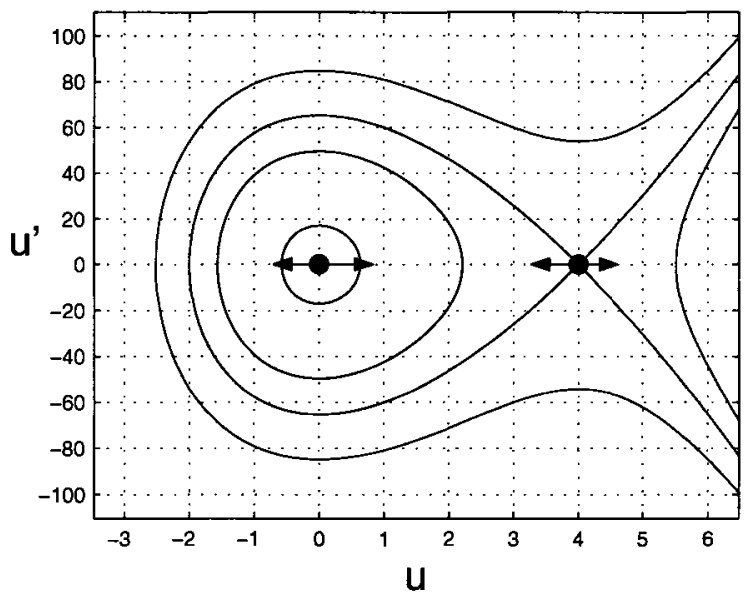

Figure 34: Phase plane of the KdV equation for fixed forcing $C=0$. As we allow the forcing to vary, the phase plane shifts, producing the slow oscillation in the nondispersive solutions.

\subsection{The Asymptotic Analysis}

In this section, we quickly rederive the dispersive solutions of the KdV equation based on the work of Amundsen, Cox, and Mortell [2]. Since the methodology is largely encapsulated in our analysis of the $\mathrm{mKdV}(3.4)$, we need only state the relevant results.

\section{Setting up the Method of Multiple Scales}

First, we will apply a multiple-scales approach to the forced KdV equation,

$$
\gamma u_{x x}+\mu u_{x}=\frac{\alpha}{2} u^{2}-(F(x)+c) .
$$

By introducing the two time scales,

$$
u(x) \rightarrow u(x, X),
$$


where $x$ is the usual slow scaling within the layer and the fast scaling $X$ is defined by,

$$
X=\frac{\omega_{1}(x)}{\sqrt{\gamma}}+\omega_{2}(x)+\sqrt{\gamma} \omega_{3}(x)+\ldots
$$

\section{The Leading Order Equations}

With $u$ now an independent function of both $x$ and $X$, the differential operators $\frac{d}{d x}$ and $\frac{d^{2}}{d x^{2}}$ need to be written out properly using the chain rule. They are found in of Section 3.4 in equations (26) and (28), respectively. Now using a perturbation expansion,

$$
u(x, X)=u_{0}(x, X)+\sqrt{\gamma} u_{1}(x, X)+\gamma u_{2}(x, X)+\ldots
$$

in the $\mathrm{KdV}$ equation (77) yields the first two orders,

$$
\mathcal{O}(1): \quad \omega_{1}^{\prime 2} u_{0 X X}-\frac{\alpha}{2} u_{0}^{2}+F(x)+c=0
$$

and

$$
\begin{aligned}
\mathcal{O}(\sqrt{\gamma}): \quad \omega_{1}^{2} u_{1 X X}-\alpha u_{0} u_{1}= & -2 \omega_{1}^{\prime} \omega_{2}^{\prime} u_{0 X X}-2 \omega_{1}^{\prime} u_{0 x X}-\omega_{1}^{\prime \prime} u_{0 X} \\
& -\frac{\mu}{\gamma} \omega_{1}^{\prime} u_{0 X}^{2} \\
\equiv & R_{1}
\end{aligned}
$$

As before, it will be useful to think of the higher order equations as,

$$
\mathcal{O}\left(\gamma^{k / 2}\right): L\left(u_{k}\right)=R_{k}, \quad k=1,2,3, \ldots
$$

where we have defined the operator $L$ by,

$$
L \equiv\left(\omega_{1}^{\prime}\right)^{2} \frac{\partial}{\partial X^{2}}-\alpha u_{0}
$$




\section{The Leading Order Dispersive Solutions}

Algebraically, the solutions to the leading order equation (81) are more easily solved than those for the corresponding $\mathrm{mKdV}$ equations. They are well known in the literature, and can be expressed in terms of as cnoidal functions,

\section{Jacobi Elliptic Dispersive Solutions (KdV)}

$$
u_{0}(x, X)=d+a \mathrm{cn}^{2}(k(X-\delta(x)), m),
$$

where,

$$
a=-8 \omega_{1}^{\prime 2} m^{2} k^{2}, \quad d=\frac{8}{3} \omega_{1}^{\prime 2} k^{2}\left(2 m^{2}-1\right), \quad k=\left(\frac{3(F(x)+c)}{16 \omega_{1}^{\prime 4}\left(m^{4}-m^{2}+1\right)}\right)^{\frac{1}{4}}
$$

\section{Avoiding Secularity and the Solvability Condition}

We now enforce a constant period on the leading order solutions (83) and derive the solvability condition using the $\mathcal{O}(\sqrt{\gamma})$ terms in equation (82). By properties of the Jacobi Elliptic functions, the period is,

$$
T=\frac{2 K(m)}{k}=4 K(m) \omega_{1}^{\prime}\left(\frac{m^{4}-m^{2}+1}{3(F(x)+c)}\right)^{\frac{1}{4}}
$$

which we set to $T=4 / 3^{1 / 4}$ for convenience. Now we can express,

$$
\omega_{1}^{\prime}=\frac{1}{K(m)}\left(\frac{F(x)+c}{m^{4}-m^{2}+1}\right)
$$

Leaving us with only one parameter to be determined: the modulus-squared $m$. Like the $\mathrm{mKdV}$, the second order equation,

$$
L\left(u_{1}\right)=R_{1}
$$

contains $u_{0 X}$ as a homogeneous solution, and so by the Fredholm Alternative, we require the right hand side, $R_{1}$ to be orthogonal to $u_{0 X}$. This yields, 


$$
\begin{aligned}
0 & =\int_{0}^{T} u_{0 X} R_{1} d X \\
& =\frac{d}{d x} \int_{0}^{T} \omega_{1}^{\prime} u_{0 X}^{2} d X-\frac{\mu}{\gamma} \int_{0}^{T} \omega_{1}^{\prime} u_{0 X} d X \quad \text { (By periodicity) } \\
& =\frac{d Q}{d x}+\frac{\mu}{\gamma} Q
\end{aligned}
$$

after making use of the periodicity of $u_{0}$ and letting $Q=\int_{0}^{T} \omega_{1}^{\prime} u_{0 X}^{2} d X$. Equation (85) is recognized as the well-known adiabatic invariant, and we can state the solvability condition in the convenient form,

\section{Solvability Condition (Damped KdV)

$$
Q(x, m(x))=\omega_{1}^{\prime} \int_{0}^{T} u_{0 X}^{2} d X=\kappa e^{-\frac{\mu}{\gamma} x}
$$

where $\kappa$ is a constant of integration and will be determined through the matching conditions. Because in this case, the analytical solutions to the $\mathrm{KdV}$ are not quite as convoluted as the rational expression of Jacobi Elliptic functions we had obtained for the mKdV, the integral in the solvability condition (85) can actually be integrated exactly (see [9]), yielding,

$$
\frac{16(3)^{1 / 4}}{5} \frac{(F(x)+c)^{5 / 4}}{\left(m^{4}-m^{2}+1\right)^{1 / 4}}\left(2 E(m)-\frac{\left(1-m^{2}\right)\left(2-m^{2}\right)}{m^{4}-m^{2}+1} K(m)\right)=\kappa e^{-\frac{\mu}{\gamma} x}
$$

\section{Matching Inner and Outer Layers}

Once the location of the matching layers $x=\tilde{x_{1}}, \tilde{x_{2}}$ is determined, the inner and outer layers can be matched as in our discussion for the mKdV (Section $3.6)$.

(i) To determine the solvability constant, $\kappa$ in (85), we require $u(x) \rightarrow u_{+}$ as we leave the inner layer, $X \rightarrow \pm \infty$. This requires $m \rightarrow 1$ as $X \rightarrow \pm \infty$ 
and consequently, we are approaching the homoclinic orbit (see Figure 34) as we arrive back at the fixed point $u_{+}$.

(ii) The modulus-squared $m(x)$ and fast coordinate scaling $\omega_{1}(x)$ are now related to the spatial variation $x$ by (84) and (85).

(iii) The phase shift $\delta(x)$ determines whether there can be a successful match on the other side of the layer $x=\tilde{x_{2}}$. Numerically, this can be calculated using a shooting-type algorithm.

(iv) Finally, we apply the mean condition (76) to determine the appropriate value of the c constant.

\section{Discussion}

The derivation of leading order dispersive solutions for both $\mathrm{KdV}$ and mKdV equations can be seen to be quite similar and in fact, these methods have been used to study more general differential equations (See Kuzmak [25], Luke [26], and Kevorkian [23]).

This concludes our asymptotic analysis. In the next section, we will compare the numerical solutions of the $\mathrm{KdV}$ and $\mathrm{mKdV}$ and discuss the accuracies of our leading-order approximations.

\subsection{The Numerical Solutions}

In this section, we present the numerical solutions of the forced KdV equation,

$$
-\gamma u_{x x x}+\alpha u u_{x}+\Delta u_{x}-\mu u_{x x}=f(x)
$$

where we have chosen the parameters as,

\begin{tabular}{|c|c|}
\hline Parameter & Value \\
\hline \hline$\gamma$ & 0.005 \\
\hline$\alpha$ & 2 \\
\hline$\mu$ & 0.0015 \\
\hline$f(x)$ & $-\pi \sin (\pi x)$ \\
\hline
\end{tabular}


Of particular interest is the plot of resonance curves shown in Figure 35, where the norm of the solution, $\|u\|_{2}$, is plotted as a function of the detuning from resonance, $\Delta$. We now describe a selection of qualitatively different solutions labeled on the figure of resonance curves:

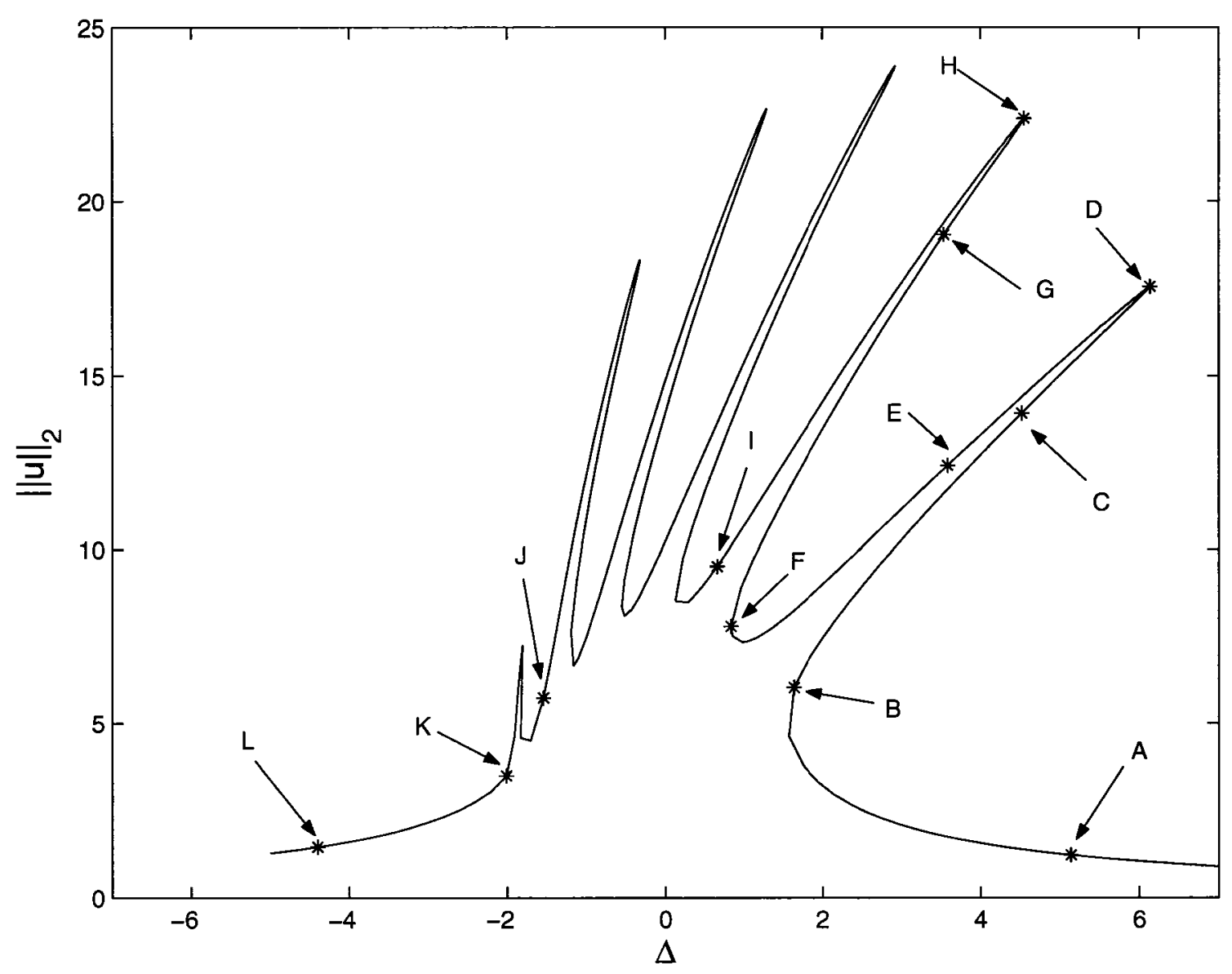

Figure 35: Resonance curves of the KdV equation. The letters A through L indicate the different regions which we address in Section 4.5.

\section{- Regions A to B}

Here, in the edges of the graph where $\Delta>\Delta^{*}$, the non-dispersive solution $u_{+}$ satisfies the mean condition and there is no need to take in account dispersive 
effects. In Figure 36, the numerical solution is shown, and the $u_{+}$curve is nearly indistinguishable. However, as $\Delta$ tends to $\Delta^{*}$, a cusp begins to form at the minimum of the non-dispersive solution. In 'response' the system begins to develop a dispersive regime, characterized by the formation of a single peak, seen in Figure 37 where the peak is centred about the minimum of $u_{+}$.

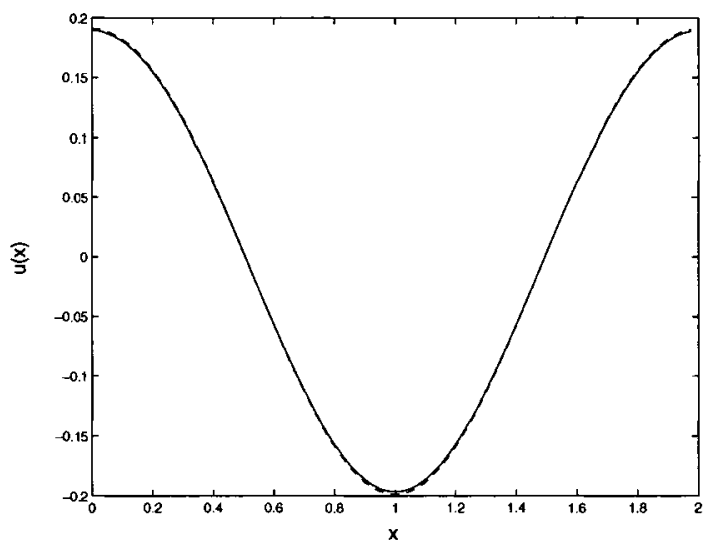

Figure 36: Region A: Valid nondispersive solutions $u_{+}$.

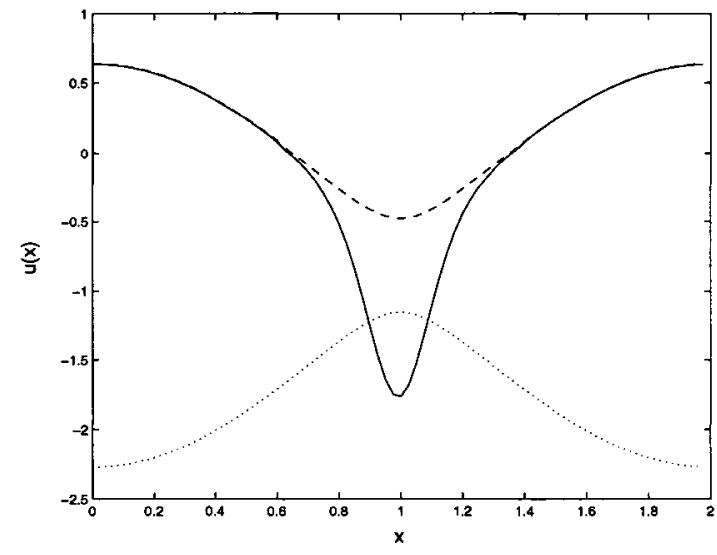

Figure 37: Region B: Beginning of dispersive regime.

\section{- Regions C to $\mathrm{E}$}

Now as we travel up the first finger, the dispersive peaks begin moving from the minimum to the maximum of $u_{+}$as a result of the exponential term in the solvability condition (85), as shown in Figure 38. At the tip of the finger in region $\mathbf{D}$, Figure 39, the dispersive peak is now positioned at the point of inflection of $u_{+}$. It will then continue its journey towards the maximum as shown in Figure 40 in region E.

\section{- Region F}

Now at the bottom between the first and second fingers, the solution contains one dispersive peak, centred about the maximum of $u_{+}$. But here, the characteristic cusp begins developing once more on $u_{+}$, and thus begins the formation of a second dispersive peak centred about the minimum where the cusp was about to form. Figure 41 illustrates the initial formations of the peak. 


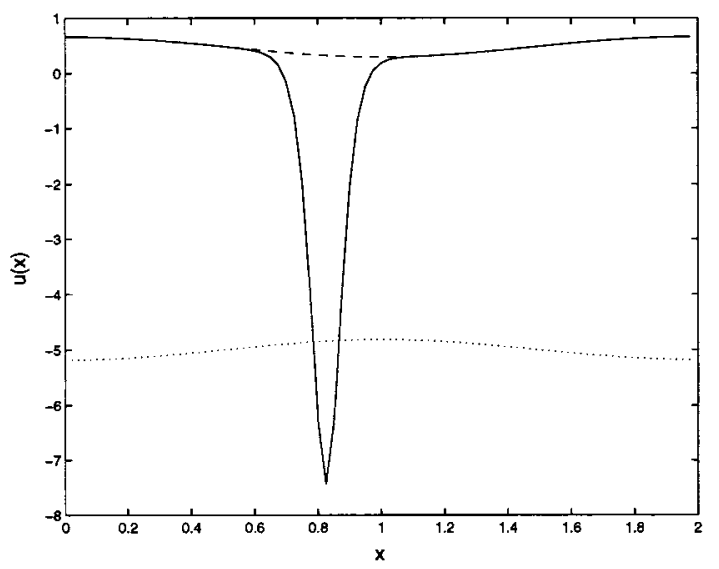

Figure 38: Region C: Underside of first finger and peak moving left.

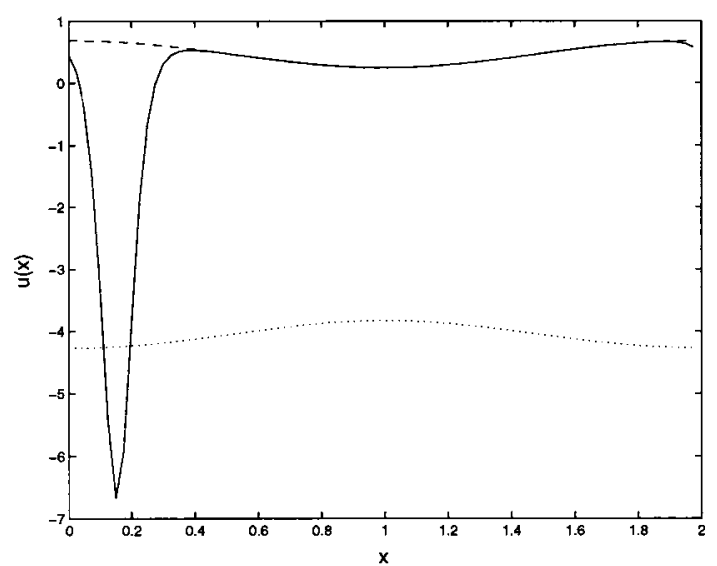

Figure 40: Region E: Top of first finger and peak moving towards maximum.

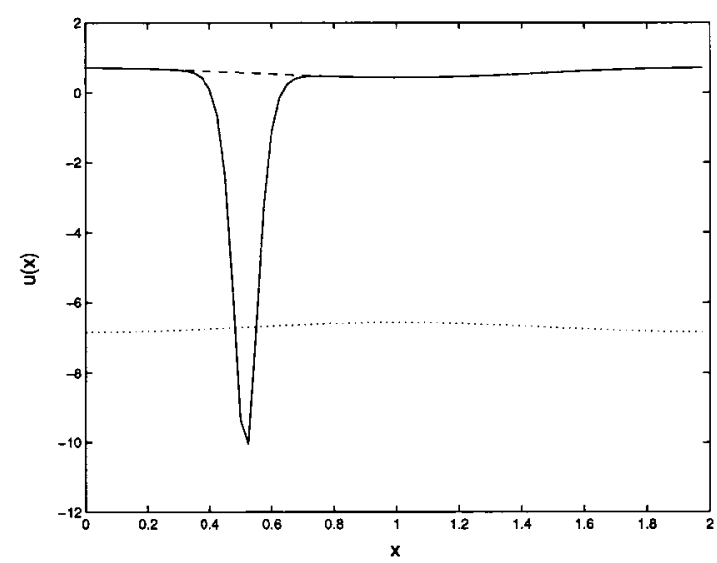

Figure 39: Region D: Tip of first finger and peak centred at point of inflection.

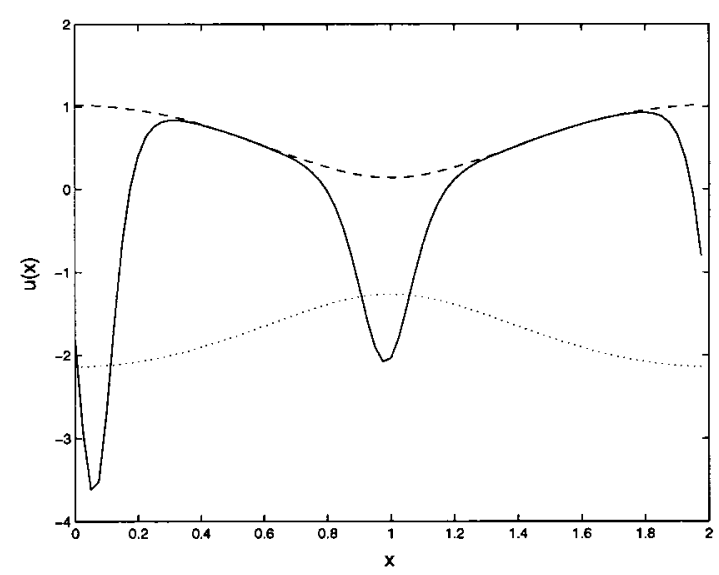

Figure 41: Region F: Between fingers and a new peak develops. 


\section{- Regions G to J, and Beyond}

Region G, located on the underside of the second finger is similar to region $\mathbf{C}$ on the first finger, with one difference: there are now two dispersive peaks. The newly formed peak near the minimum of $u_{+}$begins shifting towards the maximum as usual, but the original peak shifts instead in the opposite direction. Figure 42 illustrates this, as both peaks move towards each other. This behaviour continues until region $\mathbf{H}$ and Figure 43, where both peaks have now coalesced at the point of inflection. And now as we follow the plot down the top of the second finger in region I, Figure 44 both peaks move as one towards the maximum once more until a new peak forms at the first signs of a cusp see Figure 45.

This process repeats. That is, a peak forms at the local minimum, then shifts towards the point of inflection, where it coalesces with the previously-formed peaks. From there, they move as a group once more towards the maximum.

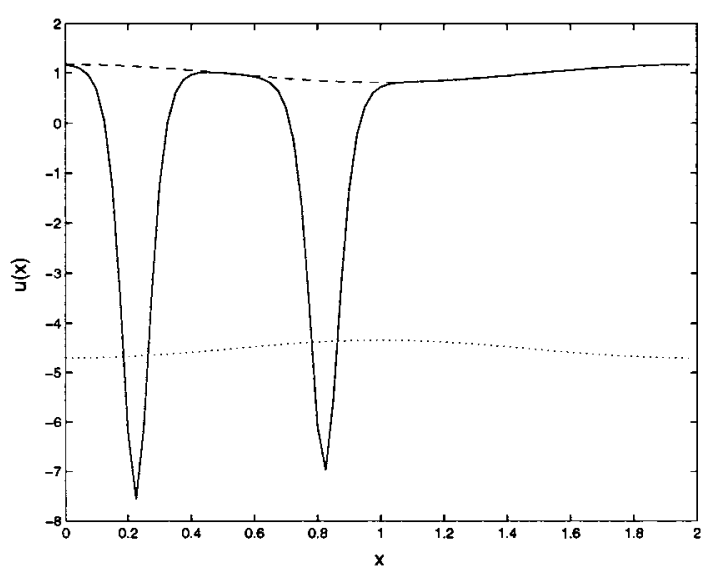

Figure 42: Region G: Underside of second finger and peaks move towards one another.

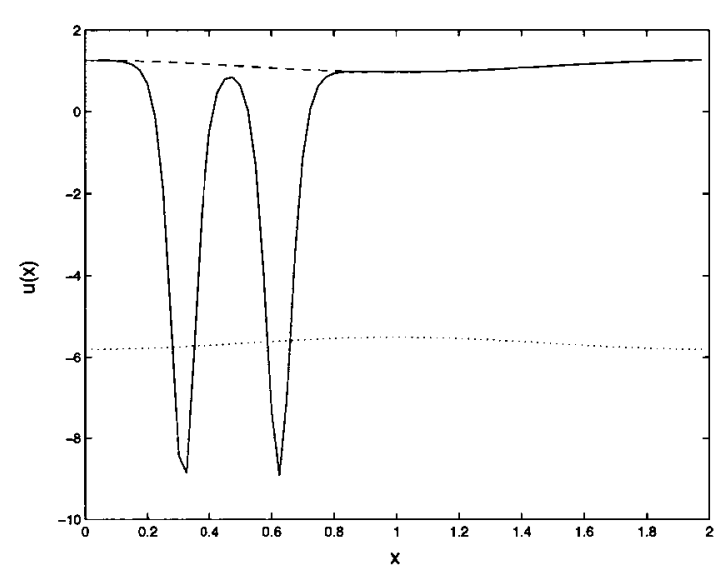

Figure 43: Region H: Tip of second finger and peaks coalesce at point of inflection.

\section{- Regions K to $\mathbf{L}$}

But this process of peak formation does not continue forever. We can explain this restriction in an ad-hoc manner: there is a constraint on the minimum width 


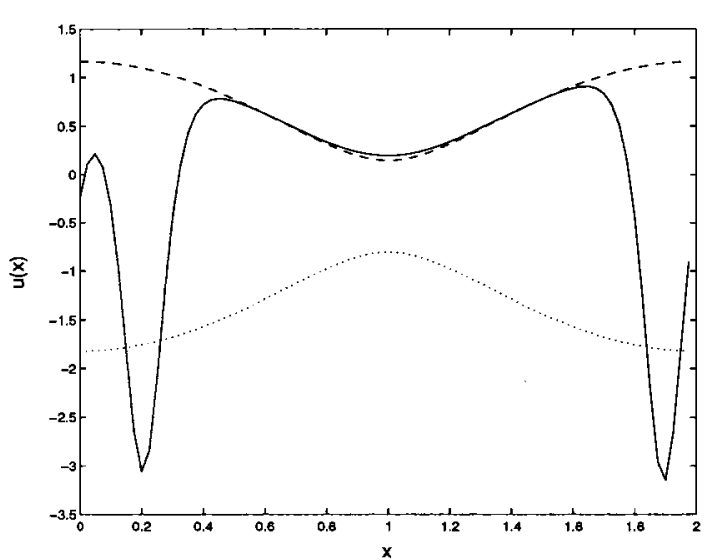

Figure 44: Region I: Between second and third fingers and just prior to the formation of a third peak.

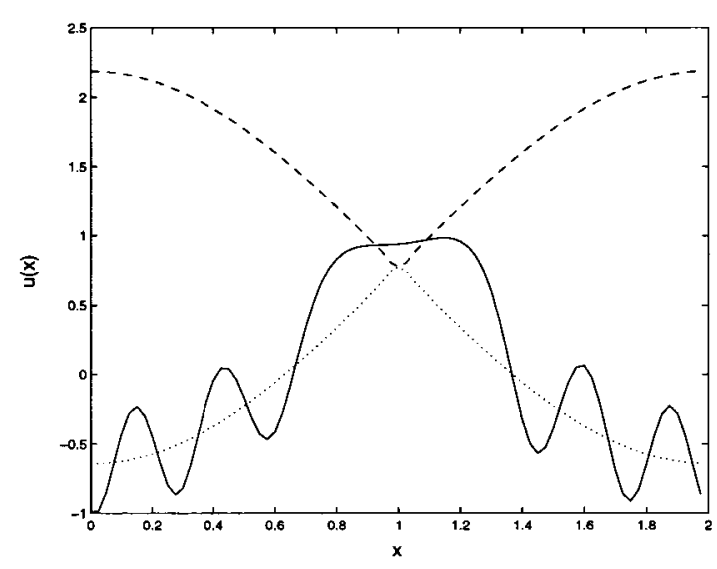

Figure 45: Region J: Peaks are beginning to overcrowd.

of the dispersive peaks because of the mean condition and consequently, the number of peaks can only increase until there is no space left for the formation of a new peak. This is shown in Figure 46 for region $\mathbf{K}$. As the domain fills up entirely with peaks, the solutions begin a natural transfer towards the second non-dispersive solution $u_{-}$. From then onwards, the size of the peaks slowly diminish until all that remains is the non-dispersive solution $u_{-}$, in the regions $\Delta<-\Delta^{*}$. This is shown in Figure 47 for region $\mathbf{L}$, where the leading order approximation is simply $u_{-}$. This process of peak overcrowding and shifting is discussed in more details in [4].

Figure 48 provides an example of the accuracy of the asymptotic approximation in comparison with the numerical solutions for the KdV equation in (86) with $\Delta=0.22$.

\subsection{The mKdV and the $\mathrm{KdV}$}

Now that we've established the main characteristics of both the KdV resonance curves above and those of the $\mathrm{mKdV}$ in Section 3.7.1, we can discuss the key differences.

\section{Non-Dispersive Solutions as Leading Order}




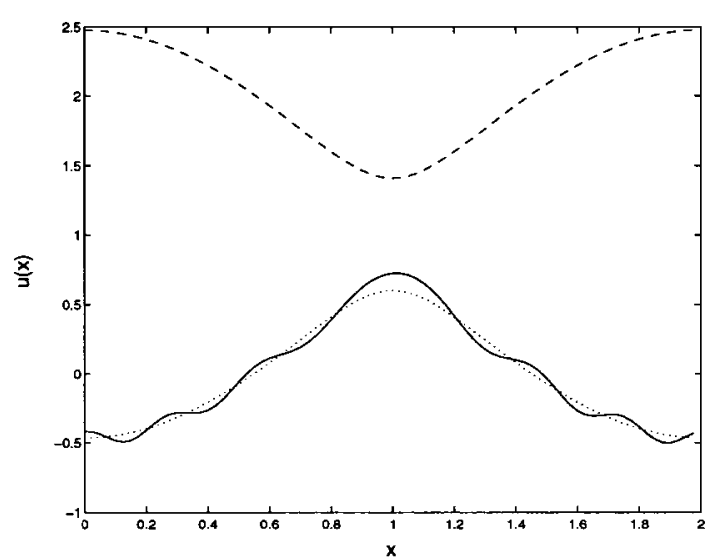

Figure 46: Region K: Peaks have transitioned into $u_{-}$

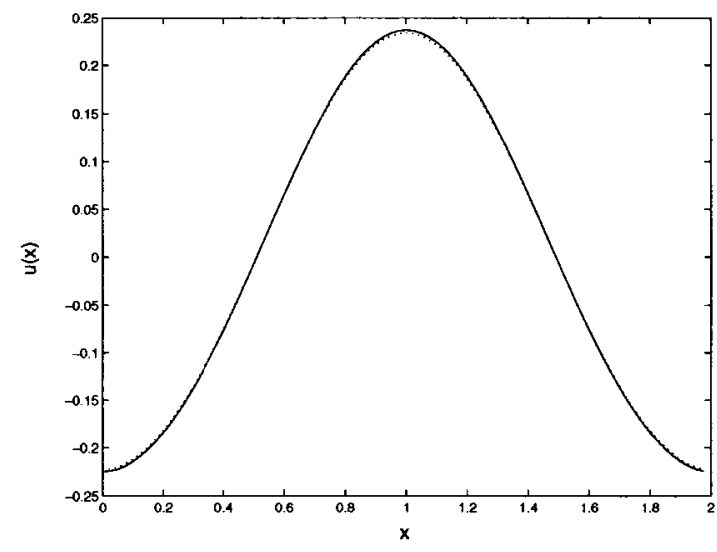

Figure 47: Region L: Valid nondispersive solution $u_{-}$

When examining the phase plane (see Figures 14 and 34), we see that two of the non-dispersive solutions of the KdV and $\mathrm{mKdV}$ share a correspondence: $u_{+}$ $(\mathrm{KdV})$ and $u_{1}(\mathrm{mKdV})$, and $u_{-}(\mathrm{KdV})$ and $u_{3}(\mathrm{mKdV})$ in our previous notation (see (15) and (78)). In both cases, these refer to the centre and the positive saddle in the phase plots. The third non-dispersive solution in the mKdV $u_{2}$ can be thought of as originating from $-\infty$, its effect only felt when it is sufficiently near to the centre $u_{3}$. As a result, the tails of the resonance curves of both KdV and $\mathrm{mKdV}$ are both formed by the comparative non-dispersive solutions.

\section{Validity of Non-Dispersive Solutions}

However, when examining the existence of the non-dispersive solutions, this is where differences can be seen between $\mathrm{KdV}$ and $\mathrm{mKdV}$. Recall that the creation of fingers on the resonance curves can be thought of as a mechanism counteracting the tendency for the non-dispersive solutions to vanish (for example, via a cusp). However, one of the fundamental differences between a cubic and a quadratic equation is the number of roots that may exist.

In particular, the cubic equation may have one or three roots $^{1}$, while the quadratic has either zero or two roots. The effect of this is to change the

\footnotetext{
${ }^{1}$ the degenerate case of two roots is not relevant because the system is continually perturbed by the forcing
} 


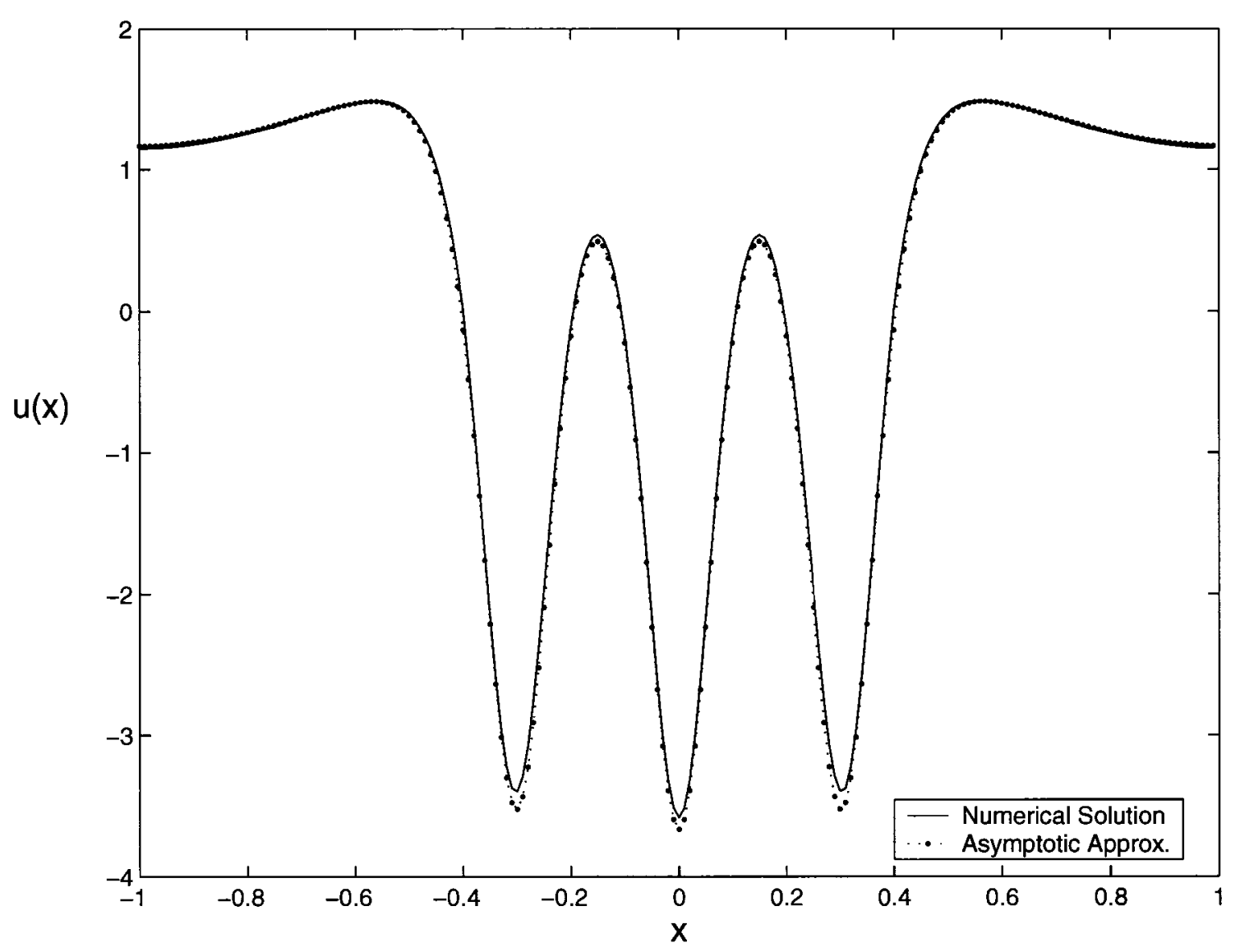

Figure 48: Comparison of numerical solution to analytical approximations for $f(x)=$ $-\pi \sin (\pi x), \gamma=0.005, \mu=0.0015$, and $\Delta=0.22$ 
types of transitions that can occur. Since both non-dispersive solutions of the $\mathrm{KdV}$ will disappear in tandem (via a cusp), solutions cannot transition between non-dispersive solutions. However, in the case of the $\mathrm{mKdV}$, there is now the distinct possibility of transitioning between non-dispersive solutions. This was shown in Section 3.2.2 and is particularly evident in Figure 13.

\section{Location of the Transition}

Let us examine the KdV and $\mathrm{mKdV}$ equations with zero damping. We saw that in the undamped equations, the location of the dispersive regions was either about a maxima or minima of the non-dispersive solution (KdV) or about a point of inflection $(\mathrm{mKdV})$. Equivalently, the dispersive peaks were either centred about a max/min of the forcing $F(x)+c$ in the case of the $\mathrm{KdV}$, or where $F(x)+c=0$ in the case of the mKdV. What accounts for the difference in positions?

In the case of the $\mathrm{KdV}$, there is an obvious symmetry in the problem when the damping is removed. The requirement is that dispersive solutions must leave and return at the same value on the non-dispersive solution - and so they are centred about a maximum or minimum of such solutions. To explain the transition about the inflection point for the $\mathrm{mKdV}$, examine the limiting dispersive transition via the heteroclinic orbit, as shown in Figure 14. This transition corresponds to a single shock transition, which we analyzed in detail using the tanh extensions in Section 3.8. By symmetry, we expect the phase plane to contain this heteroclinic orbit when the solution is in the centre of the layer - at the very top of the orbit, where the effects of both saddles are equal. This corresponds to exactly the case $F(x)+c=0$ and the cubic roots are symmetric, explaining the position of the dispersive layer about the point of inflection. 


\subsection{Summary}

- The crucial difference between the $\mathrm{KdV}$ and $\mathrm{mKdV}$ can be seen in the phase planes: The quadratic KdV possesses zero or two real roots. In contrast, the $c u$ bic $\mathrm{mKdV}$ possesses zero, one, or three real roots. As a result, $\mathrm{mKdV}$ solutions can (and will) transition between roots, while $\mathrm{KdV}$ solutions can not.

- In the undamped case, the boundary layers of the KdV equation occur at the $\max / \mathrm{min}$ of the non-dispersive solutions, while for the $\mathrm{mKdV}$, they occur at the points of inflection. 


\section{Chapter 5}

\section{The Extended Korteweg-de Vries Equation}

\subsection{Introduction}

The extended Korteweg-de Vries equation (eKdV) is the final piece of the puzzle, and will complete our study of the three KdV-type equations:

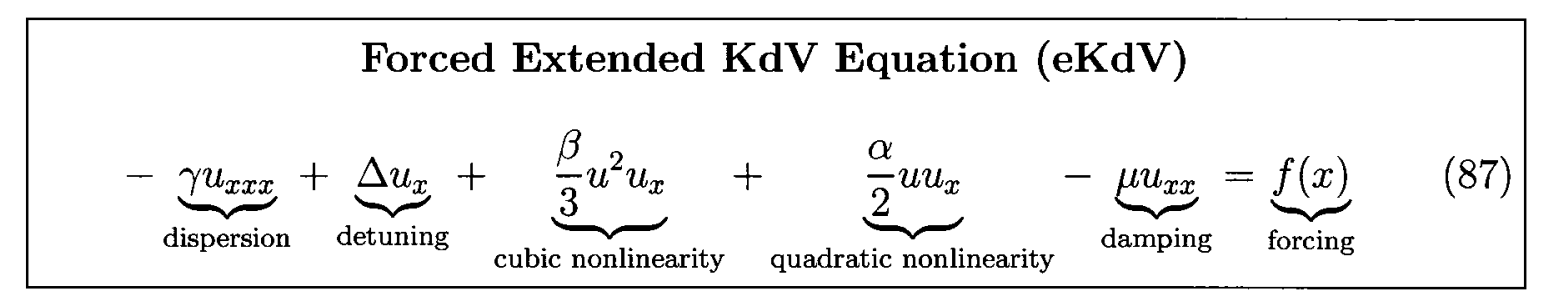

The eKdV combines the cubic nonlinearity seen in the mKdV (chapter 3 ) with the the quadratic nonlinearity seen in the regular KdV (chapter 4). For clarity, we will only refer to the eKdV when both cubic and quadratic nonlinearities are present $(\alpha, \beta \neq 0)$, and refer to the special cases with only one nonlinear term as the KdV or $\mathrm{mKdV}$ as usual.

The uniqueness of our work in this section derives from our interpretation of the $\mathrm{KdV}$ spectrum, which contains the $\mathrm{KdV}$ and $\mathrm{mKdV}$ variants on either end (see Figure 49). Having studied the resonant solutions of the $\mathrm{mKdV}$ and $\mathrm{KdV}$ equations 


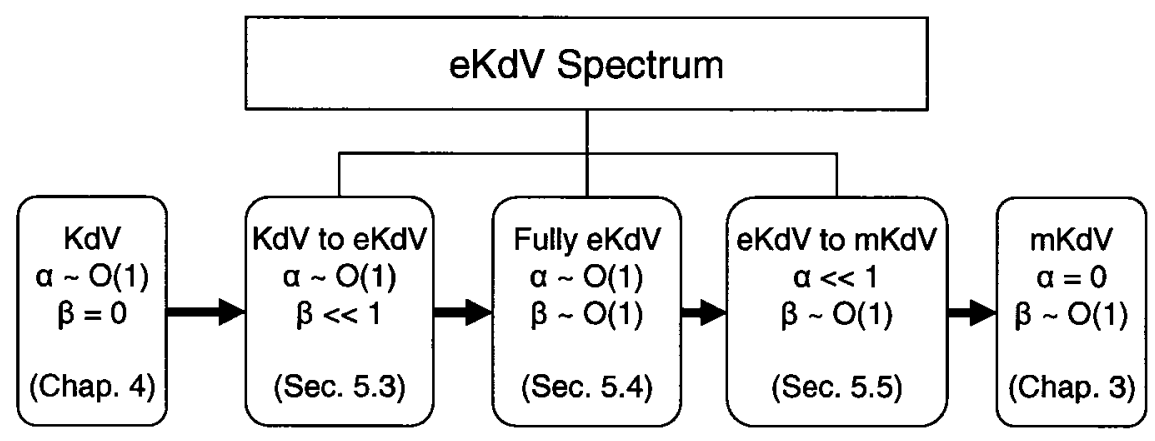

Figure 49: Figure of the eKdV spectrum and our plan of attack.

in Chapters 3 and 4, we will attempt to tie together the two special classes.

This KdV-eKdV-mKdV connection has not been established in the literature. In most cases, the research has been solely focused on studying only one specific class of the KdV family, perhaps spurred by a particular physical system. In other cases, authors have reduced one system into another (e.g. through a re-scaling), such as in the work of Marchant and Smyth [28], but the end result is still that only one of the three $\mathrm{KdV}$ variants is studied in any detail.

Thus, there is a definite lack of study concerning the eKdV equation, examining the effects as the nonlinearity transitions from purely quadratic $(\mathrm{KdV})$ to purely cubic ( $\mathrm{mKdV}$ ), and with a combined regime in between. Our goal is to unify these three important classes of $\mathrm{KdV}$ equations. We begin Section 5.2 by describing a useful transformation between the $\mathrm{eKdV}$ and $\mathrm{mKdV}$ equations. Essentially, this allows us to defer any analytic questions of the eKdV to our work on the mKdV in chapter 3. However, we are primarily concerned with rather the qualitative aspects of the solutions and this will be the focus of the rest of the chapter.

In Section 5.3, we will examine the transition from KdV to eKdV equations. This will continue in Section 5.4 where we examine the eKdV equation when the quadratic and cubic factors are of equal importance (which we call, the 'fully' eKdV). Finally, in Section 5.5 we examine the transition from $\mathrm{eKdV}$ to $\mathrm{mKdV}$ equation, where the 
cubic nonlinearity dominates. Figure 49 presents a useful guide to our plan of attach in this chapter.

\subsection{A Transformation from eKdV to $\mathrm{mKdV}$}

Akin to the linear transformation presented in Section 4.2.1 of chapter 4 that allows us to remove the detuning parameter of the KdV equation, there is a similar transformation for the eKdV which removes the quadratic nonlinearity.

If we begin with equation (87) and perform a linear shift using,

$$
v=u+\frac{3 \alpha}{4 \beta},
$$

the $\mathrm{eKdV}$ equation is recast as an $m K d V$ equation,

$$
-\gamma v_{x x x}+\beta v^{2} v_{x}+\bar{\Delta} v_{x}-\mu v_{x x}=f(x),
$$

where now the detuning parameter changes to,

$$
\bar{\Delta}=\Delta-\frac{3 \alpha^{2}}{16 \beta} .
$$

But now the mean condition also changes to,

$$
\int_{0}^{2 L} u(x) d x=\int_{0}^{2 L}\left(v-\frac{3 \alpha}{4 \beta}\right) d x=0
$$

or,

$$
\int_{0}^{2 L} v(x) d x=\frac{3 \alpha}{2 \beta} L .
$$

Thus at least in theory, asymptotic solutions to the eKdV equation can be treated using the mKdV theory of Chapter 3. Unfortunately, this transformation does not lend itself to any significant grasp of the qualitative structure the eKdV solutions. Having changed the mean condition (91), the class of allowable solutions also changes and so the particular details of the asymptotic matching or types of boundary layer behaviour will need to be dealt with separately. 
Although this will be our last encounter with the transformation, we should remain aware that at least on an analytical level, the eKdV is no different from the mKdV. However qualitatively, we will later see that solutions can be similar to both those of the $\mathrm{KdV}$ and the $\mathrm{mKdV}$. In the next section, we will examine how the eKdV spectrum behaves, focusing instead on the qualitative details.

\subsection{The Transition from $\mathrm{KdV}$ to $\mathrm{eKdV}$}

The transition that occurs when we begin with a KdV equation with leading order nonlinearity,

$$
-\gamma u_{x x x}+\alpha u u_{x}+\Delta u_{x}-\mu u_{x x}=f(x)
$$

where $\alpha \sim \mathcal{O}(1)$ and introduce a cubic factor,

$$
-\gamma u_{x x x}+\beta u^{2} u_{x}+\alpha u u_{x}+\Delta u_{x}-\mu u_{x x}=f(x),
$$

where $\beta \ll 1$ is the focus of this section.

We shall see that this particular regime ties together many of the different properties observed during our study of the mKdV and KdV equations and also serves to answer some of the questions we may have left open in previous chapters. We can begin by sketching the cubic polynomials of an undamped KdV and an eKdV with small cubic nonlinearity,

$$
\begin{aligned}
& \text { (1) : } \quad \gamma u^{\prime \prime}=\frac{\alpha}{2} u^{2}+\Delta u-C \\
& \text { (2) : } \quad \gamma u^{\prime \prime}=\frac{\beta}{3} u^{3}+\frac{\alpha}{2} u^{2}+\Delta u-C
\end{aligned}
$$

where we have chosen the parameters as follows, 


\begin{tabular}{|c|c|}
\hline Parameter & Value \\
\hline \hline$\gamma$ & 0.005 \\
\hline$\alpha$ & 2 \\
\hline$\beta$ & 0.1 \\
\hline$\Delta$ & -6 \\
\hline$C$ & 0 \\
\hline
\end{tabular}

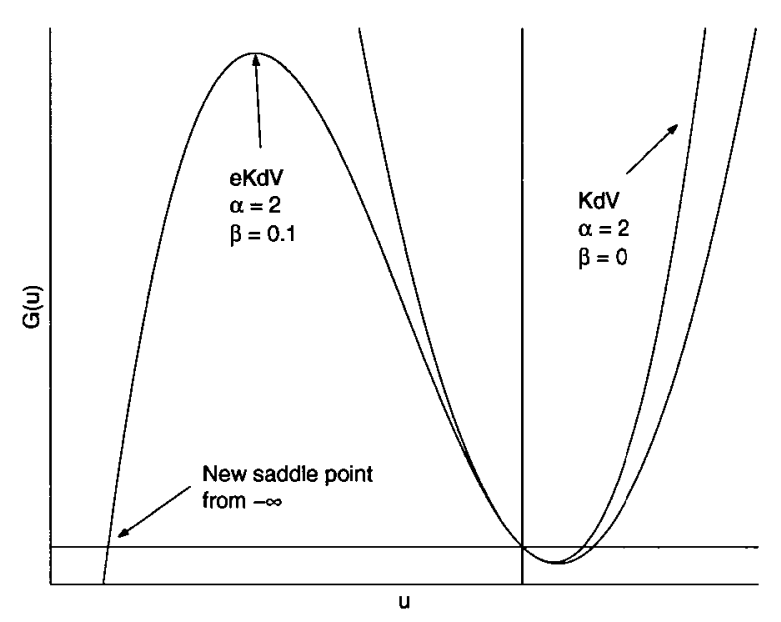

Figure 50: The cubic polynomials of a KdV and weakly cubic eKdV. Notice the creation of a new saddle as well as the introduction of a small asymmetry.

Figure 50 illustrates these two cubic equations. Remember that the roots of the cubic equations reveal the locations of the non-dispersive solutions for a fixed value of the spatial variation $x$. We can see the two effects on the non-dispersive solutions after introducing the cubic factor: First, a newly created third solution, tending from $u=-\infty$ begins to approach the other two non-dispersive solutions as larger and larger values of $\beta$ are chosen. This third solution corresponds to $u_{2}$ in our previous notation for the mKdV (See equation (15)).

However, we would also expect that the effect of this change on the pre-existing solutions is minimal, especially when the old solutions are sufficiently far from the newly formed root. Furthermore, the introduction of the cubic factor changes the 
centre root (corresponding to $u_{-}$in the $\mathrm{KdV}$ and $u_{3}$ in the $\mathrm{mKdV}$ ) very little while the right root (corresponding to $u_{+}$in the $\mathrm{KdV}$ and $u_{1}$ in the $\mathrm{mKdV}$ ) - if it exists is moved closer to the centre.

We can thus differentiate two possible consequences, depending on the relative size of the cubic nonlinearity $\beta$ : regimes where the newly formed root is largely ignored by the solution trajectories (at leading order), and regimes where the cubic root can not be ignored. We summarize our observations so far:

- New saddle point from $-\infty$

Introducing a cubic factor produces a second saddle to the left of the centre fixed point.

- Small changes in symmetry of pre-existing solutions

The original centre and saddles found in the KdV do not change too dramatically for small $\beta$.

\section{- Possible cubic attraction}

Certain large-amplitude solutions may tend close to new saddle. This is where the mKdV-type behaviour may arise.

\subsubsection{No Substantial Cubic Effect}

As an example when the KdV type behaviour dominates throughout the resonant band, examine Figure 51, which overlays the resonance curves of our equations (93) and (94), that is, the KdV equation (1) with $\alpha=2$, and the eKdV (2) with $\alpha=2$ and $\beta=0.1$.

The noteworthy qualitative difference is a subtle change in the slope of the fingers: the fingers bend towards the left when the cubic factor is introduced. This is simply a result of the new asymmetric attraction between the non-dispersive solutions with now the trajectories in the phase plane spend a little more or less time near one of the three non-dispersive solutions. 


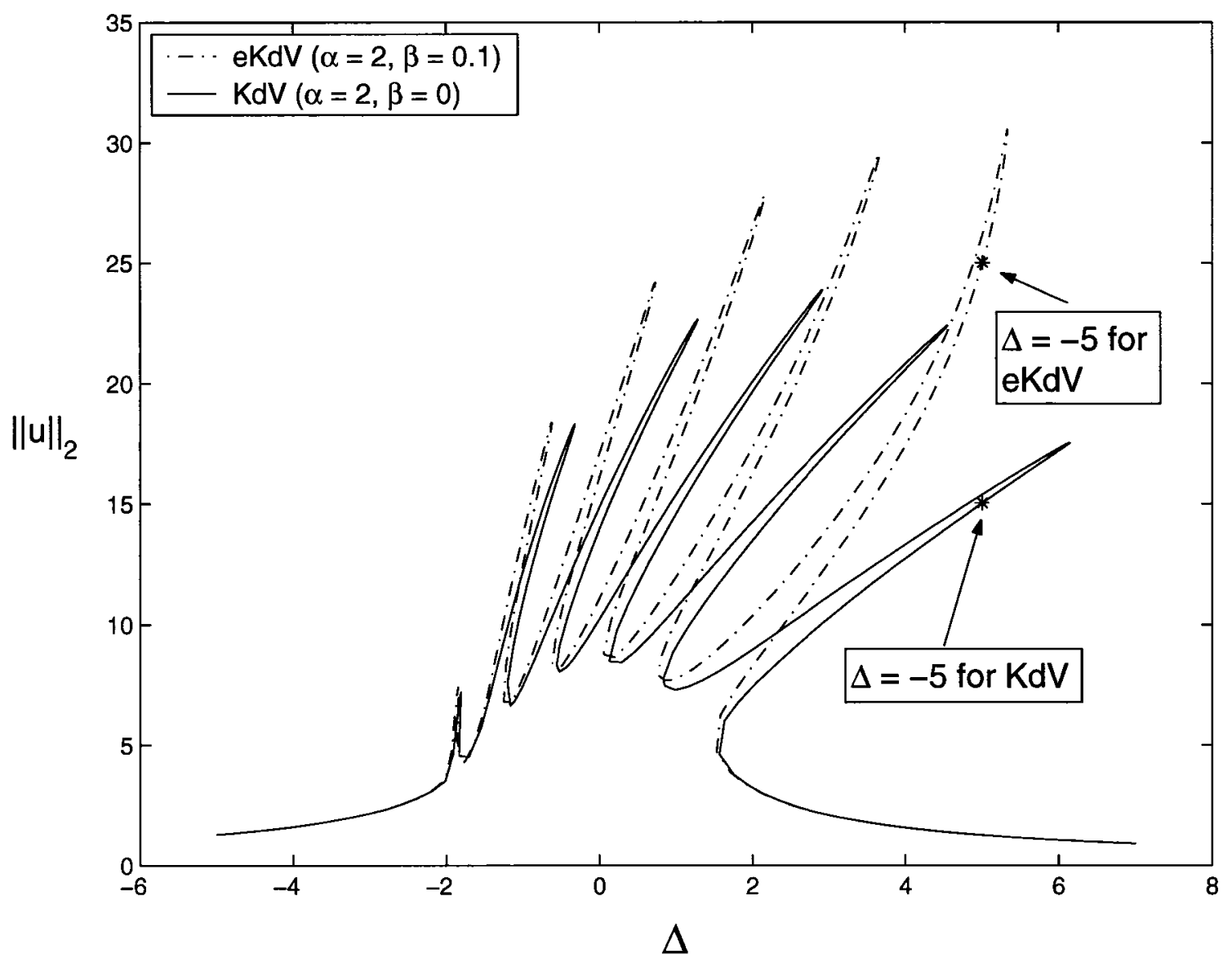

Figure 51: Resonance curves of the $\mathrm{KdV}$ and weakly cubic eKdV equations. The indicated points are discussed in the text. 
In this prime example of an eKdV equation purely dominated by the quadratic $\mathrm{KdV}$-type behaviour, the assumption that,

$$
\mathcal{O}(\beta) \sim \mathcal{O}(\gamma)
$$

and the typical perturbation (or multiple-scales) expansion,

$$
u(x)=u_{0}(x)+\sqrt{\gamma} u_{1}(x)+\gamma u_{2}(x)+\ldots,
$$

removes the negligable cubic effect and re-scales the equation so that our KdV theory in Chapter 4 can be readily applied. This is similar to the work of Marchant and Smyth during their study of the eKdV equation in [28]. Figure 52 illustrates the solutions for $\Delta=5$ on the corresponding locations of the resonance curves shown in Figure 51.

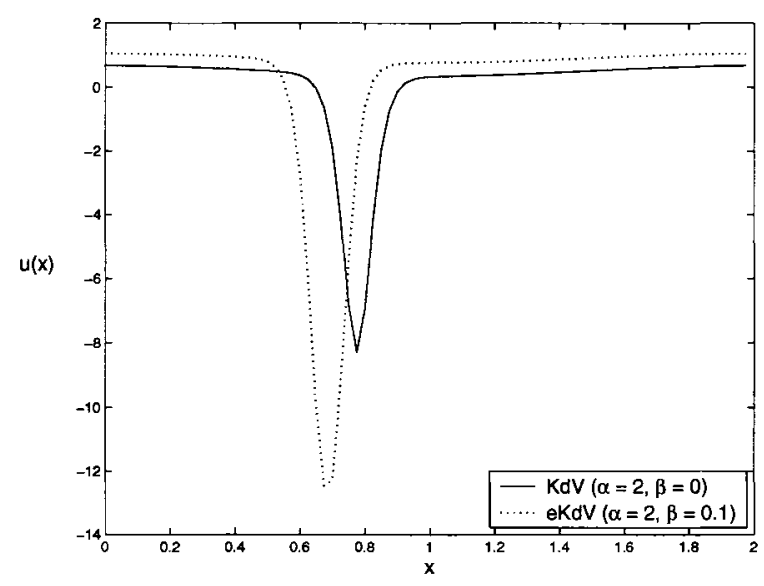

Figure 52: A comparison between solutions of the $\mathrm{KdV}$ and weakly cubic eKdV at $\Delta=-5$. Although the solutions are quite different in size, their overall shape remains the same.

Notice that both solutions contain the same $\operatorname{sech}^{2}$ type profile, thus the analytic framework for the $\mathrm{KdV}$ in Chapter 4 should remain reasonably similar for this case as well. However, the larger $\beta$ is relative to $\alpha$, the more prominent the differences in phase and peak amplitude. What happens when at leading order, the solutions 
are no longer dominated by the quadratic nonlinear term? This is the question we examine in the next section.

\subsubsection{Substantial Cubic Effect}

We can surmise that in certain large-amplitude solutions, the phase plane behaviour may tend close to the newly introduced cubic root, thus producing more mKdV-like behaviour than before. In fact, this separation between $\mathrm{KdV}$ and $\mathrm{mKdV}$-type regimes occurs at a singular point.

This sudden change is illustrated in Figure 53, where the resonance plot of the $\mathrm{KdV}$ equation (1) with $\alpha=2$ is shown, along those for two other $\mathrm{eKdV}$ equations of the form (2), this time with $\beta=0.1035$ and $\beta=0.1037$.

As seen, the smaller cubic factor of $\beta \approx 0.1035$ serves to bend the fingers back and upwards but qualitatively, there is still little difference in the solutions. However, near this critical value, the finger suddenly shoots backwards if $\beta$ is increased any further, as in the resonance plot for the $\mathrm{eKdV}$ with $\beta \approx 0.1037$. This is reminiscent of what was observed in the mKdV equation (Compare with Figure 20). Qualitatively, what accounts for this sudden change?

Remember that in the $\mathrm{KdV}$ regime, the first fingertip in the resonance plot indicates a location where the dispersive peak is centred upon the inflection point of the non-dispersive solutions (See Figure 39).

To be more specific, consider the sequence of solutions displayed in Figure 54, corresponding to the $\mathrm{eKdV}$ equation with smaller cubic nonlinearity, $\beta=0.1035$.

- Region E ( $\Delta=4.8$ in Figure 54 and Figure 55)

As we move from left to right on topside of the first finger of Figure 53, the dispersive peak moves rightward from the maximum of the non-dispersive solution to the point of inflection. Accompanying the increasing norm $\|u\|_{2}$ is an increasing amplitude in peak height.

- Region D $(\Delta=5.26$ in Figure 54 and $\Delta=5.24$ in Figure 55) 


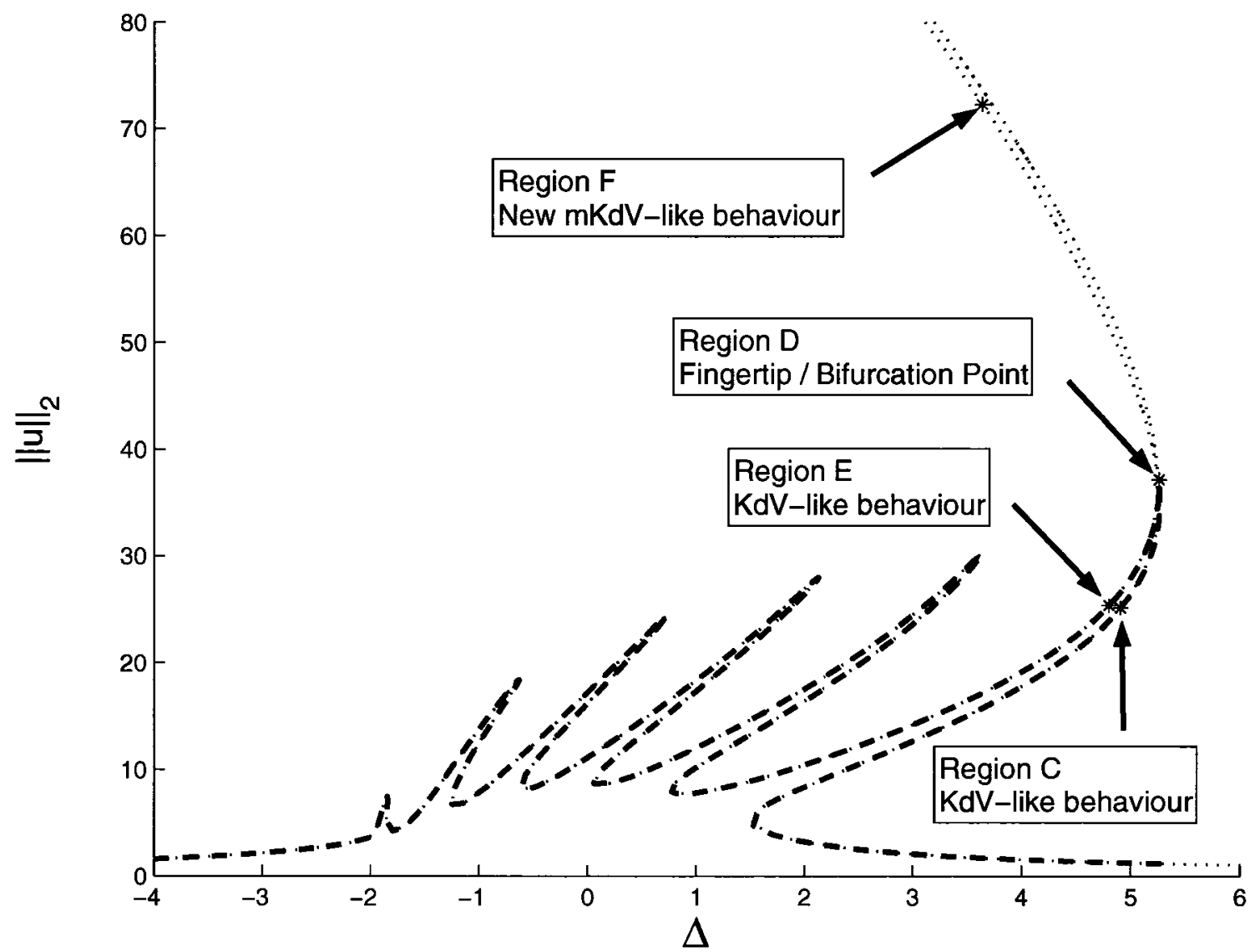

Figure 53: Resonance curves of the eKdV equation 


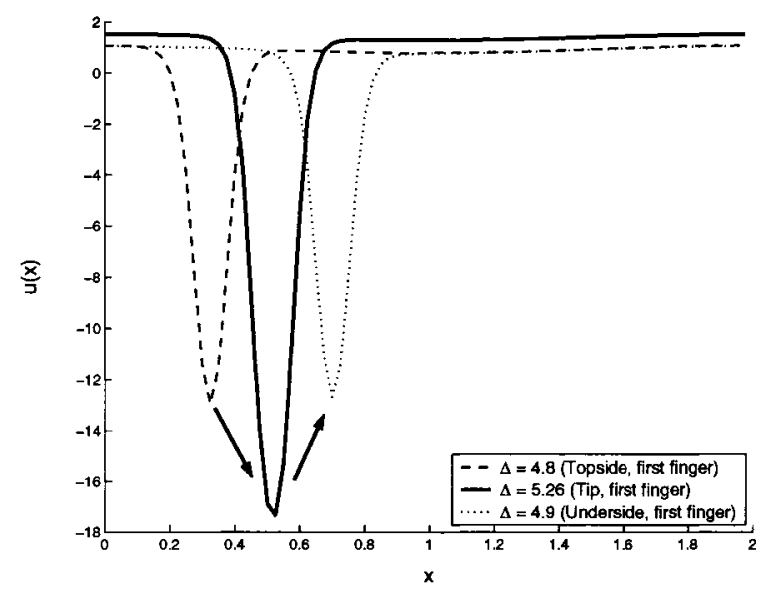

Figure 54: Insufficient cubic effect and thus the behaviour is KdV-like.

Once the peak has reached the point of inflection at the tip of the finger where the amplitude is a maximum, a decision is made: depending on the amplitude of the dispersive peak and consequently, on $\beta$, the solution may proceed back down the finger into Region $\mathbf{C}$ or may change dramatically, finding itself instead in Region F.

- Region C $(\Delta=4.9$ in Figure 54)

If the peak is too far from the third non-dispersive solution, the KdV-type behaviour continues as usual; the peak continues moving rightward, down the finger, as both the norm $\|u\|_{2}$ and the peak amplitude decreases $(\Delta=3.64)$. The shift continues until the peak is positioned at the minimum, and the peak quickly vanishes (Compare to Regions A through D in Figures 36-39 in Section $4.5)$.

- Region F ( $\Delta=3.64$ in Figure 55)

However, if the peak is sufficiently close to the third non-dispersive solution, then a new type of behaviour emerges. The sequence shown in Figure 55 depicts this radical change. As before, the peak moves towards the point of inflection as we climb the topside of the first finger (Region E). However, at this critical value 
of $\beta=\beta^{*}$ and at the tip of the finger $\Delta=\Delta^{*}$, the maximum peak amplitude is sufficiently large so that the solution begins to be pulled towards the newly introduced cubic root (Region D).

Now, instead of continuing the rightward movement, the peak shifts in direction and proceeds backwards, all the while increasing in amplitude (Region F). This corresponds to the sudden change in the resonance curves at the tip of the finger, and the renewed increase in the norm $\|u\|_{2}$.

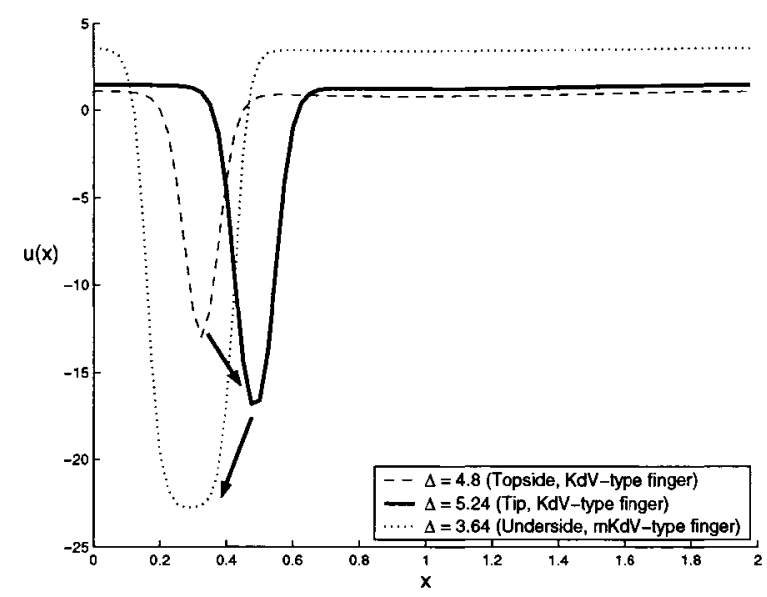

Figure 55: Sufficient cubic effect and the behaviour is mKdV-like

The creation of this new $m K d V$-like regime, though dramatic, is hardly surprising having been prepared by our investigation of the $\mathrm{mKdV}$ in Chapter 3 . Figure 56 provides the full resonance plot for the $\mathrm{eKdV}$ at the critical transition $\beta \approx 0.1037$. Figure 57 examines some of the characteristic solutions on this branch in detail. In particular, note the circular movement of the dispersive layer about the point of inflection. In the next section, we will discuss this peak movement in slightly more detail and also discuss the bifurcation point where the $\mathrm{mKdV}$-regime first appears. 


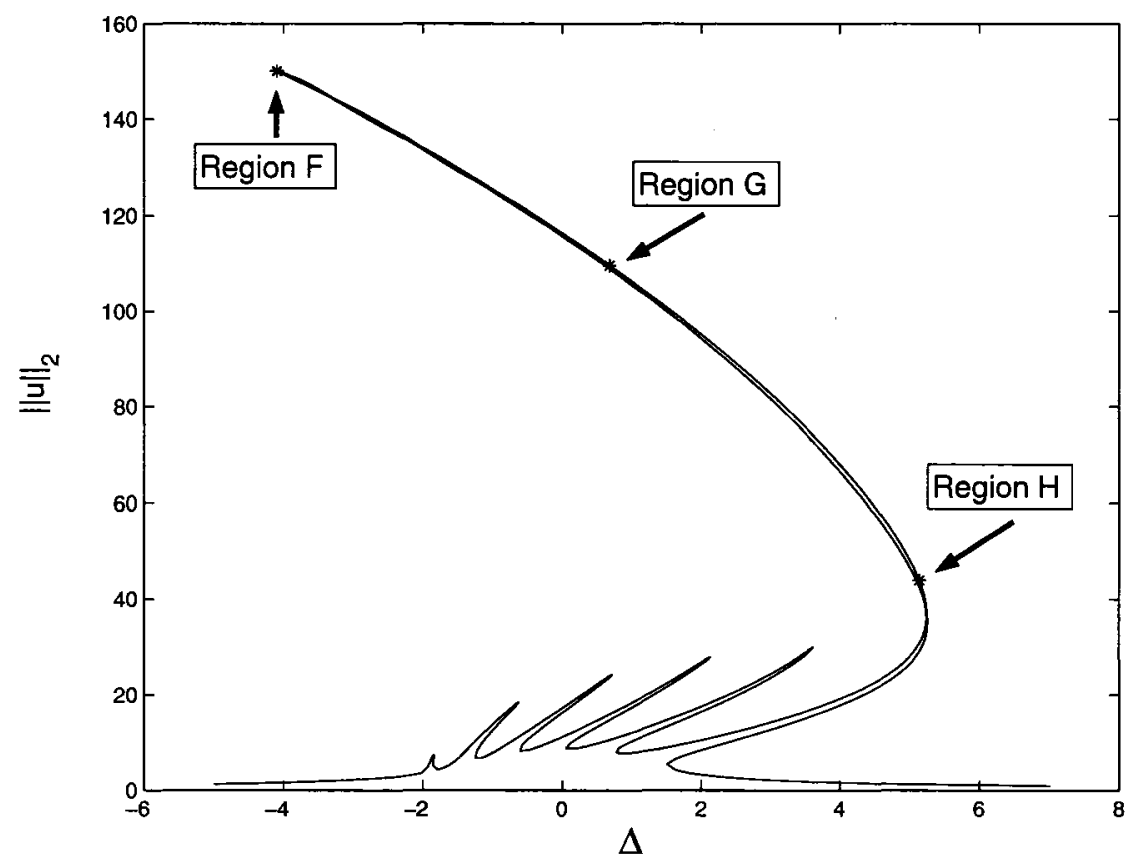

Figure 56: The full resonance curves of the eKdV equation after the bifurcation point. The region labels correspond to the solutions in Figure 57

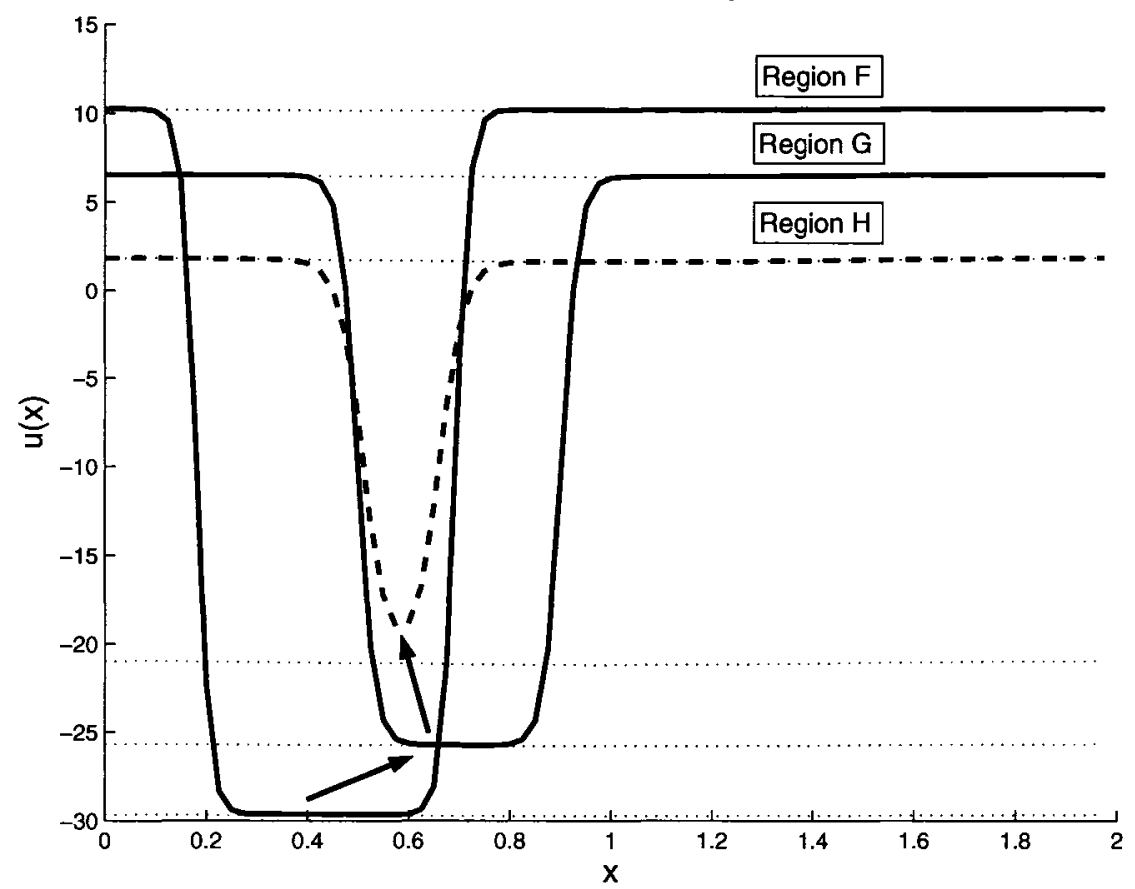

Figure 57: Solutions of the eKdV equation after the bifurcation point. The labels correspond to the regions shown in Figure 56. Notice the circular movement of the peaks. 


\subsubsection{Predicting the Bifurcation and the Peak Movement}

Unfortunately, while the location of the critical bifurcation point $\left(\beta^{*}\right.$ and $\left.\Delta^{*}\right)$ is easy to find and describe qualitatively, analytically tracking the peak movement is not an easy problem to solve. We know from our study of the $\mathrm{mKdV}$ and $\mathrm{KdV}$ equations that the location of the dispersive peaks are readily provided by the solvability condition in equations (52) or (85),

$$
Q(x, m(x))=\omega_{1}^{\prime} \int_{0}^{T} u_{0 X}^{2} d X=\kappa e^{-\frac{\mu}{\gamma} x} .
$$

For simple choices of $u(x)$, the expression can be integrated and the peak locations found by studying the extremum of the expression. However, in the case of an eKdV equation, the quartic roots involved in the dispersive solutions (37) as well as the more convoluted expression of elliptic functions makes the task of providing an explicit expression for the layer location a difficult one indeed!

For the KdV equation, Amundsen, Cox, and Mortell showed in [2] that, for the case of the $\mathrm{KdV}$ in (93) with $\alpha=\frac{3}{2}$, the location of the dispersive layers are found at $x=\delta_{1}$ and $x=\delta_{2}$ in the following expressions:

$$
\begin{gathered}
\delta_{1}=\frac{1}{\pi}\left[\arcsin \left(\frac{c}{\sqrt{1+\frac{25 \pi^{2} \gamma^{2}}{16 \mu^{2}}}}\right)+\arctan \left(\frac{4 \mu}{5 \pi \gamma}\right)\right], \\
\delta_{2}=1-\frac{1}{\pi}\left[\arcsin \left(\frac{c}{\sqrt{1+\frac{25 \pi^{2} \gamma^{2}}{16 \mu^{2}}}}\right)+\arctan \left(\frac{4 \mu}{5 \pi \gamma}\right)\right],
\end{gathered}
$$

where the principal branches of the inverse trigonometric functions are used. The full details of their work are not particularly important here, but the key observation is that the existence of two possible peak trajectories is given in the case of the $\mathrm{KdV}$ by (96) and (97).

Why are there two locations? The two expressions corresponds to either the top or underside of the fingers in the resonance curves. Thus, we can also expect $\delta_{1}=\delta_{2}$ for parameters corresponding to the fingertips of the resonance curves. 


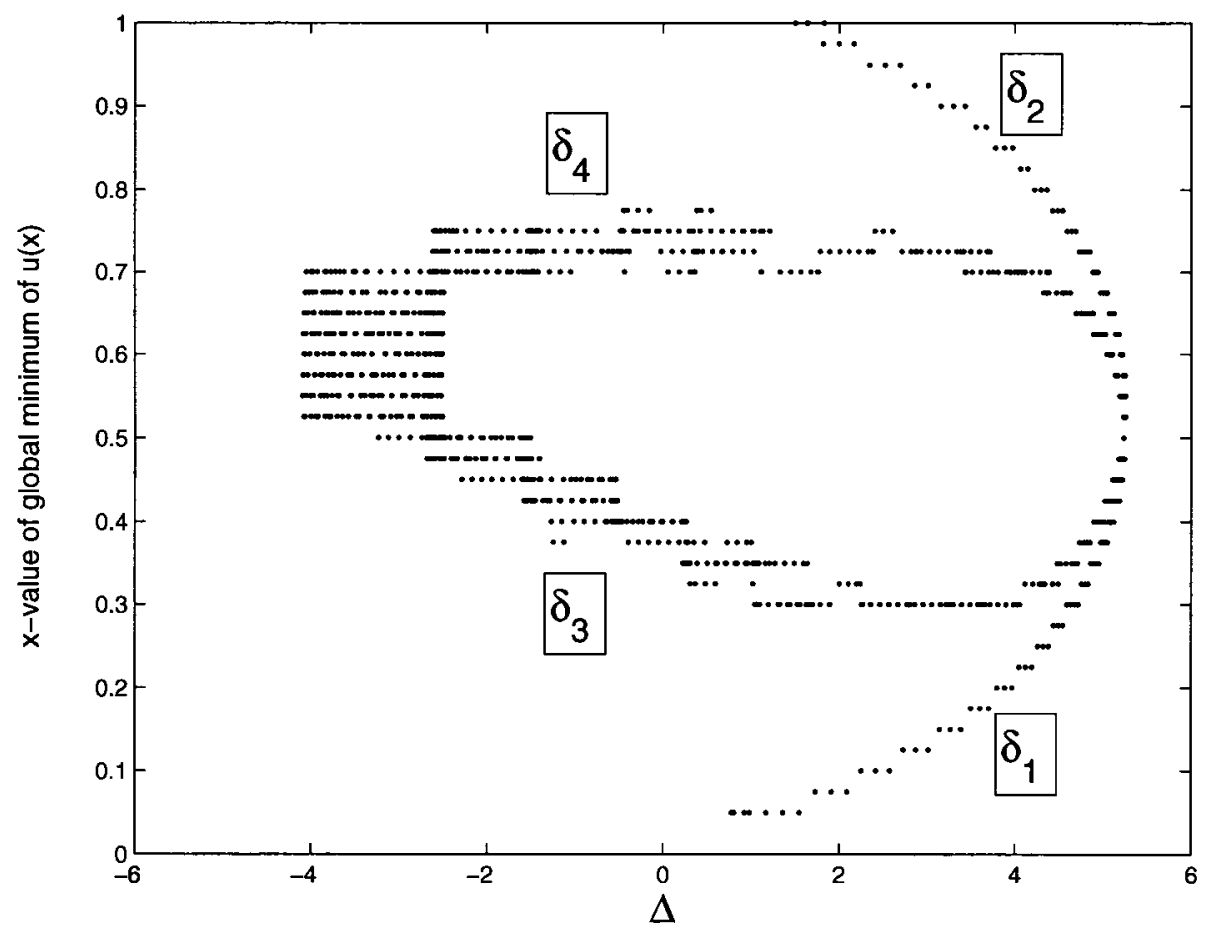

Figure 58: Tracking the movement of the dispersive peak on the first finger. $\delta_{1}$ and $\delta_{2}$ correspond to the $\mathrm{KdV}$-like regime, and $\delta_{3}$ and $\delta_{4}$ describe the position of the peak within the mKdV regime. 
Similarly, the layer locations for the eKdV equations are provided by a similar expression, but this time involving four possible solutions, $\delta_{1}$ through $\delta_{4}$. Figure 58 provides the evidence for our claim, as we track the single peak location (or global minimum) numerically for $\beta=0.1037$ as a function of $\Delta$ (the figure is restricted to only solutions in the first finger).

Within the KdV-regime (near $\delta_{1}$ and $\delta_{2}$ ), notice the monotonic shift in the dispersive layer from $x=0(\max )$ to $x=1$ (min). However, at the point of inflection $(x=0.5)$, the solutions bifurcate. Although we can continue via $\delta_{2}$ into the KdV regime, a more interesting case follows $\delta_{3}$ and $\delta_{4}$ into the $\mathrm{mKdV}$ regime. The $\mathrm{mKdV}$ regime is marked in the figure by the circular and slightly asymmetric movement of the peaks. This matches our observation of the solutions in Figure 57.

But the algebraic difficulty of the problem makes proving our claim difficult. Integrating the problem directly in (95) does not seem viable, but there is still hope of an alternate argument or simplification to track the layer movement, and consequently, the bifurcation points in the $\mathrm{eKdV}$. For the moment, we leave this question open and will briefly bring it up again in Chapter 6 . In the next section, we discuss what happens as the cubic parameter, $\beta$ is further increased, and we enter into the middle of the eKdV spectrum.

\subsection{In the Middle of the eKdV Spectrum}

Let us begin where we left off in the previous section: at the critical juncture $\left(\beta^{*}, \Delta^{*}\right)$ when the first finger bends backwards and enters an mKdV-like regime. As we continue to increase the cubic factor $\beta$, each finger from right to left, will begin to mimic the previous one's behaviour.

The resonance plots of Figure 59 displays this transition into the strongly cubic, strongly quadratic region of the $\mathrm{eKdV}$, for $\alpha=2$ and $\beta=3$. By now, the fingers have all been significantly affected by the cubic factor and have been accompanied by an increase in length, similar to what was seen in Figure 56. Note that the sixth finger is not shown, the domain of our resonance curves not wide enough to accommodate the long fifth finger. 


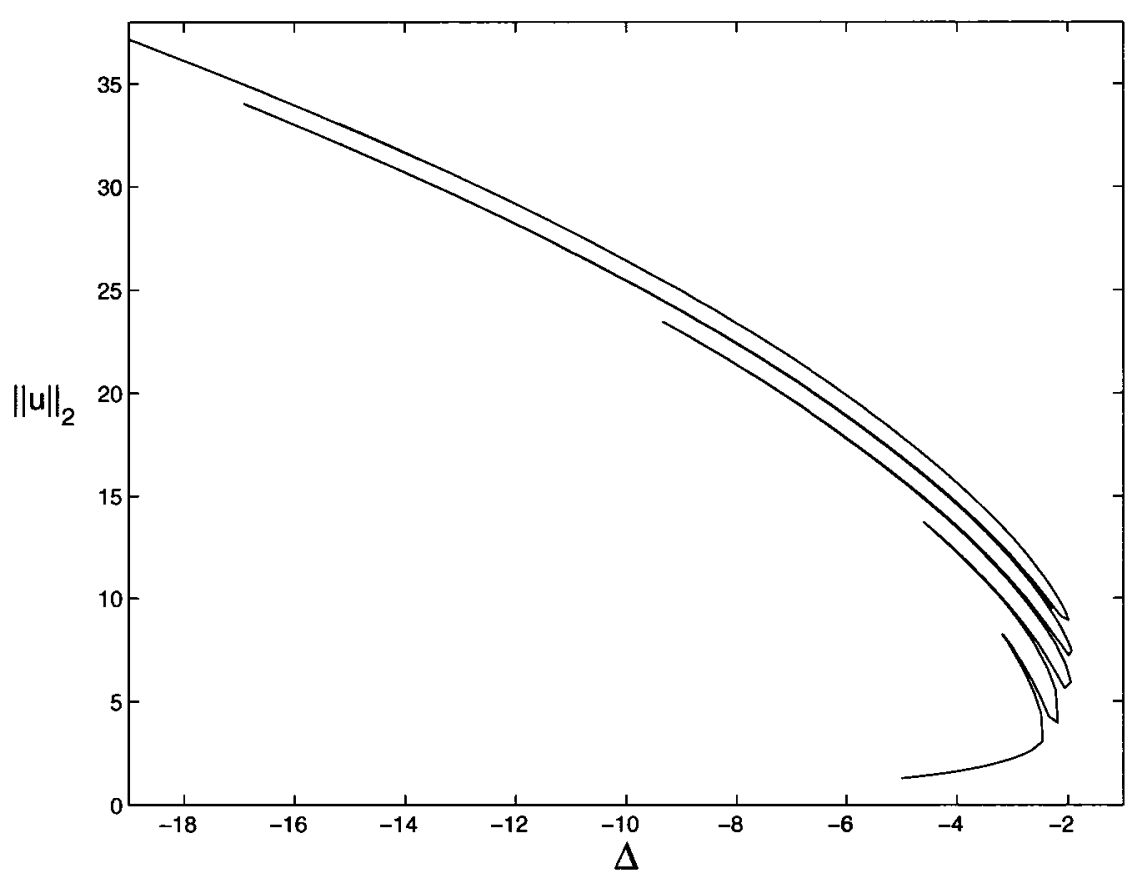

Figure 59: Resonance curves of the fully eKdV equation with $\alpha=2$ and $\beta=3$ 
In terms of the particular solutions, the boundary layer behaviour of the eKdV solutions can be roughly described as either of the KdV-type (near homoclinic transitions) or of the mKdV type (near heteroclinic transitions). The only significant difference in what we have previously seen with the $\mathrm{mKdV}$ or the $\mathrm{KdV}$ is that the quadratic factor provides an inherit asymmetry of the problem, thus mixtures of $\mathrm{KdV}$ or mKdV-type transitions can occur.

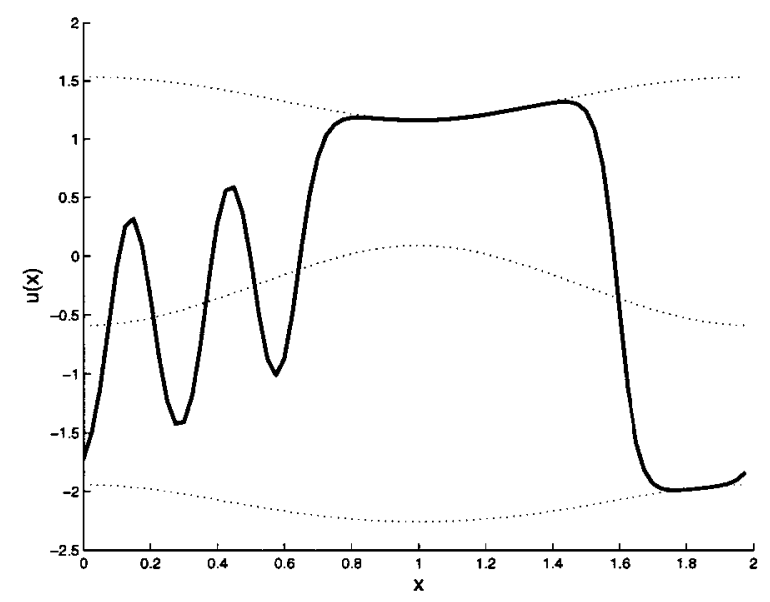

Figure 60: An example of the combined $\mathrm{KdV}$ and $\mathrm{mKdV}$ effects on a fully quadratic and cubic nonlinear $\mathrm{eKdV}$. Notice the obvious asymmetry of the boundary layers.

For example, examine Figure 60 and notice the stark asymmetry in the solution. Unlike in the $\mathrm{KdV}$ or $\mathrm{mKdV}$, there is now a possibility of different behaviour in each of the two boundary layers. This is due a combination of the damping (which moves shifts the dispersive layers) and the asymmetric position of the roots (which provides different degrees of attraction from the fixed points).

In sum, there are no surprises as to the boundary layer behaviour of eKdV solutions - they will either be reminiscent of the mKdV-type (near heteroclinic transitions between non-dispersive solutions) and/or of the KdV-type (near homoclinic transitions to and from the same non-dispersive solution). However, the intermingling of a quadratic factor (providing an asymmetry) and a cubic factor (restoring a symmetry) produces a much richer array of possible solutions. 


\subsection{The Transition from eKdV to $\mathrm{mKdV}$}

Finally, as $\beta$ becomes much larger than $\alpha$ and the quadratic factor becomes less important, the resonance curves begin to mirror those of the $\mathrm{mKdV}$ equation (see Figure 20). The crucial observation is that for large cubic nonlinearity and small (nearly negligible) quadratic nonlinearity, the only affect on the phase plane is a small asymmetric positioning of the roots, as in Figure 61 . This is also seen by the fact the linear transformation (88) from the eKdV to $\mathrm{mKdV}$ is the identity $v \approx u$ when $\beta \gg \alpha$.

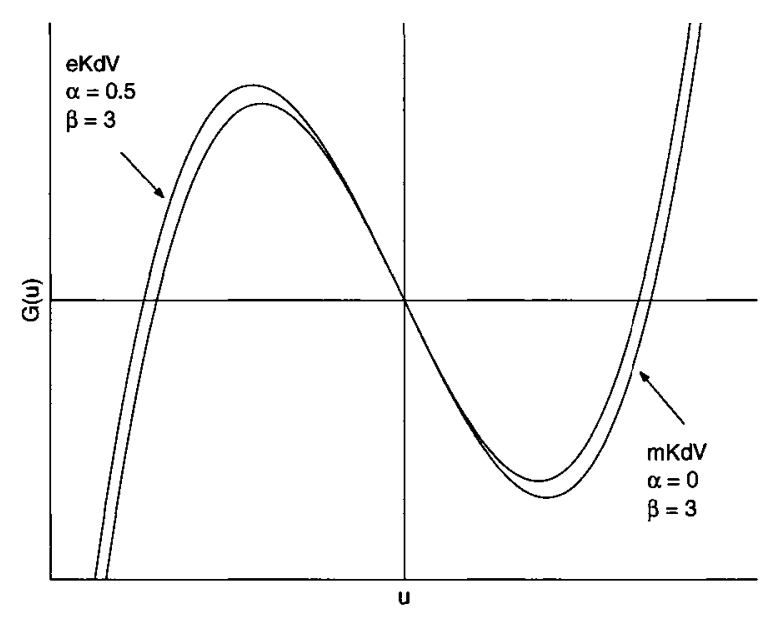

Figure 61: The cubic roots of the $\mathrm{mKdV}$ and a weakly quadratic eKdV equation.

In our first encounter with the resonance curves of the $\mathrm{mKdV}$ equation in Figure 20 , we were slightly unsure of how the resonance curves for negative $\Delta$ transitioned to positive $\Delta$. Was it a continuous transition? If so, how many fingers form? Following our discussion in the previous section, we now realize that there are indeed a total of six fingers which have fully reversed directions from the KdV equation, but they are so long in length they can not covered by our domain in Figure 35.

We have thus succeeded at completing a qualitative discussion of the eKdV spectrum and in particular, have revealed the mechanisms which unite the solutions of the $\mathrm{KdV}$ equation with those of the $\mathrm{mKdV}$ equation. In the final section of this chapter, 
we summarize our observations.

\subsection{Summary}

- The theory we have developed previously for the $\mathrm{mKdV}$ and $\mathrm{KdV}$ equations can be applied to understanding the eKdV.

- Any eKdV equation can be transformed into an mKdV equation through a linear shift.

- Introduction of a cubic factor into the $\mathrm{KdV}$ equation produces significant qualitative change at a critical point when the parameters are such that the dispersive solution is attracted to the new cubic root.

- Introduction of a quadratic factor into the $\mathrm{mKdV}$ produces little change.

- There is an interesting bifurcation point where the weakly cubic eKdV first enters an mKdV-like regime. This is characterized by a sharp change in the resonance curves. This bifurcation point can be predicted by studying the location of the layers, but the algebraic difficulty makes deriving even implicit expressions difficult. 


\section{Chapter 6}

\section{Conclusions}

\subsection{Summary of Results}

\section{Resonant Solutions of the mKdV Equation}

Analytic and numerical methods were applied to solve the steady state periodically forced $\mathrm{mKdV}$ equation with Burgers damping. Using the method of multiple scales, uniformly valid leading order approximations in the form of elliptic functions were derived and shown to accurately predict the numerical solutions for a wide range of parameters.

\section{The KdV, mKdV and eKdV Connection}

The resonant solutions of the forced eKdV equation were studied, with an emphasis on varying the quadratic and cubic nonlinearities. We showed that in general, solutions of the $\mathrm{KdV}$ can be roughly described as possessing behaviour of the KdV-type (asymmetry in the boundary layers and near homoclinic orbits) or of the mKdV-type (transitions between two non-dispersive solutions).

An intriguing bifurcation point in the eKdV was discovered where the solutions first enter an mKdV-type regime. This point is marked by a sharp change in the resonance curves (Figure 62), as the solutions encounter a new set of dispersive behaviour. 


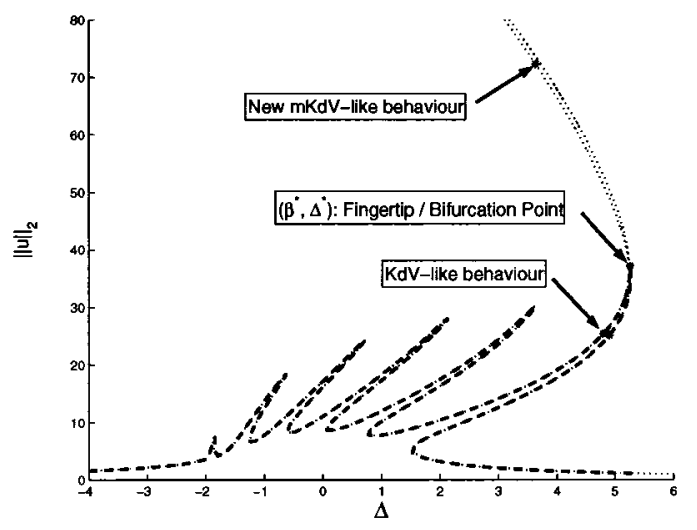

Figure 62: The ekdV bifurcation

\subsection{Summary of Contributions}

\section{A Unification of KdV-type Equations}

The most significant contribution of this thesis is in unifying the solutions and characteristics of the $\mathrm{KdV}, \mathrm{mKdV}$, and $\mathrm{eKdV}$ equations. Our work culminates in an analysis of the eKdV spectrum, seen in Figure 63, and we provide a broad overview of the similarities, differences, and connections between these equations of the KdV-type.

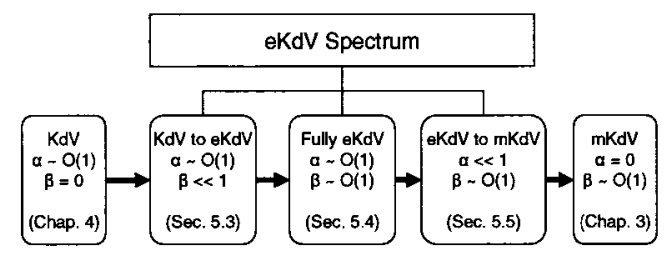

Figure 63: Studying the KdV-mKdV-eKdV connection.

\section{A Development of a Flexible Methodology}

Throughout this work, we have sought to emphasize the effectiveness and flexibility of our methodology in studying the KdV-type equations. Inspired and 
guided by the work of Amundsen, Cox, and Mortell [2], we have successfully extended their methodology to study the forced $\mathrm{mkdV}$ and $\mathrm{eKdV}$ equations. The theory is elegant, practical, and has to potential to be applied to a wide class of problems.

\subsection{Future Research}

\section{A Global Approach to Asymptotic Approximations}

While our outlined methodology is successful in developing asymptotic approximations for most solutions of the KdV-type, it is implicit in the procedure that the dispersive layers are independent and can be studied individually. But this local and layer-based approach will fail in cases where dispersive layers separate or coalesce.

So how are these solutions handled? In special cases where the dispersive layer(s) have expanded to cover the entire domain, as in Figure 64, then a simple linearization about the appropriate non-dispersive solution will work. This was done by Ockendon, Ockendon, and Johnson [33] for the periodically forced $\mathrm{KdV}$ equation.

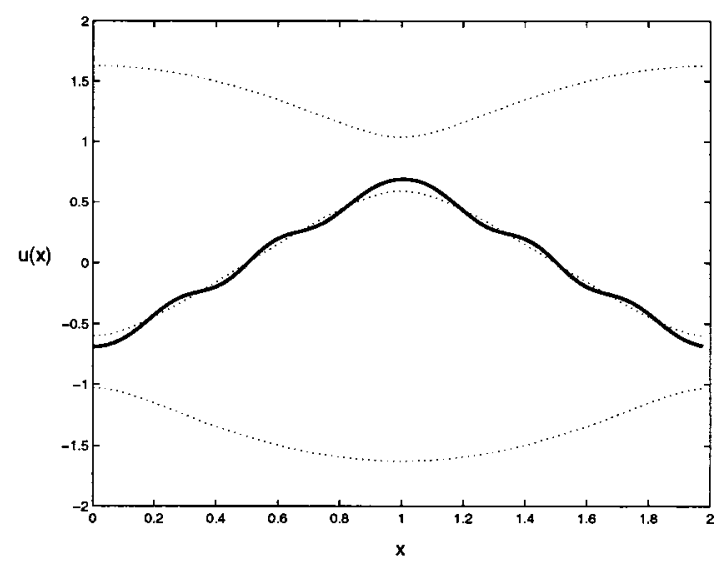

Figure 64: A solution where the dispersive layer occupies the entire domain.

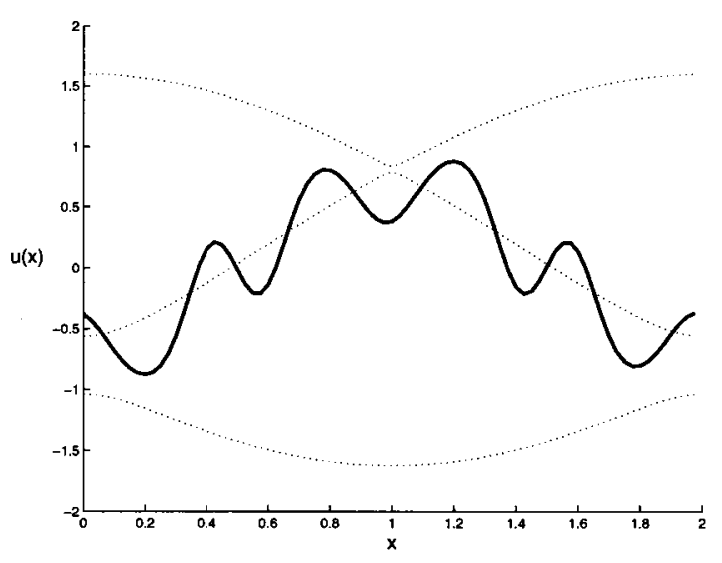

Figure 65: Are there one or two layers? A layer-based approach may not work. 
But for more complex solutions where there is no clear distinction between nondispersive and dispersive layers, such as in Figure 65, then a more global-based approach seems necessary. This is reminiscent of a WKB-type method (see [3]), where the form of the solutions are assumed a priori, and there is no need to study boundary layers. Unfortunately, conventional WKB techniques are only useful for linear equations, but there has been some effort to extend WKB theory to nonlinear equations (see for example, [30]).

\section{The Cubic and Quadratic Bifurcation in the eKdV}

One of the questions largely left unanswered in our work is the nature of the eKdV bifurcation $\left(\beta^{*}, \Delta^{*}\right)$, separating $\mathrm{KdV}$-like behaviour from $\mathrm{mKdV}$-like behaviour. It was pointed out that the bifurcation can be understood by way of the boundary layer locations $\delta_{1}$ to $\delta_{4}$, and Figure 66 provides visual evidence of this claim. However, the details remain tightly shrouded in the algebraic complexity of the problem, and we hope that a more elegant alternative to the problem exists. In any case, the nature of this bifurcation is fascinating not only mathematically, but in a physical standpoint as well, and it would be interesting to see if this critical juncture arises in physical phenomena.

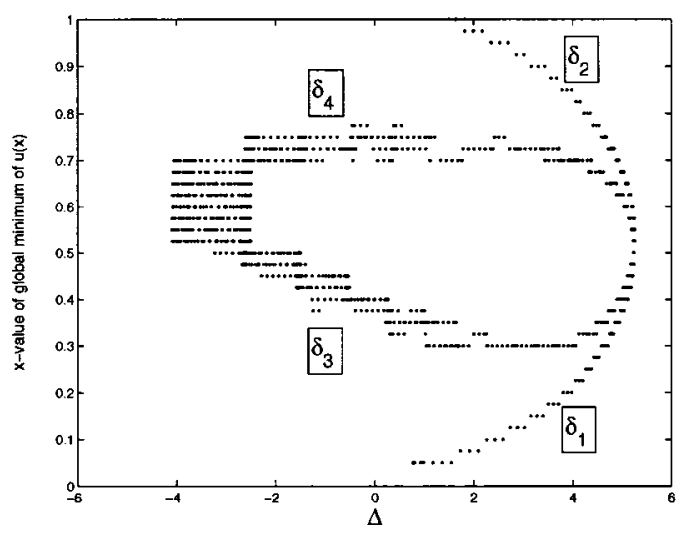

Figure 66: The bifurcation, seen by tracking the movement of the dispersive peaks. 


\section{Appendix A}

\section{Existence and Uniqueness of Solutions}

Let us begin with the steady state extended KdV equation,

$$
-\gamma u_{x x x}+\Delta u_{x}+\alpha u u_{x}+\beta u^{2} u_{x}-\mu u_{x x}=f(x) .
$$

To apply known theorems of existence and uniqueness, we would like to transform this third order differential equation into a first order system of equations as follows,

$$
\left(\begin{array}{l}
u_{1} \\
u_{2} \\
u_{3}
\end{array}\right)^{\prime}=\left(\begin{array}{c}
u_{2} \\
u_{3} \\
\frac{1}{\gamma}\left(-\mu u_{3}+\Delta u_{2}+\alpha u_{1} u_{2}+\beta u_{1}^{2} u_{2}-f(x)\right)
\end{array}\right)
$$

where $u_{i}: \Omega \rightarrow \mathbb{R}$. Thus, we simply have a system,

$$
\mathbf{u}^{\prime}=\mathbf{F}(x, \mathbf{u}(x))
$$

where $\mathbf{F}: \mathbb{R}^{4} \rightarrow \mathbb{R}^{3}$ is clearly continuous in any region $\Omega \times U \subseteq \mathbb{R}^{4}$. Also, the derivative matrix is given by,

$$
\left(\begin{array}{lll}
\frac{\partial F_{1}}{\partial u_{1}} & \frac{\partial F_{1}}{\partial u_{2}} & \frac{\partial F_{1}}{\partial u_{3}} \\
\frac{\partial F_{2}}{\partial u_{1}} & \frac{\partial F_{2}}{\partial u_{2}} & \frac{\partial F_{2}}{\partial u_{3}} \\
\frac{\partial F_{3}}{\partial u_{1}} & \frac{\partial F_{3}}{\partial u_{2}} & \frac{\partial F_{3}}{\partial u_{3}}
\end{array}\right)=\left(\begin{array}{ccc}
0 & 1 & 0 \\
0 & 0 & 1 \\
\frac{1}{\gamma}\left(\alpha u_{2}+2 \beta u_{1} u_{2}\right) & \frac{1}{\gamma}\left(\Delta+\alpha u_{1}+\beta u_{1}^{2}\right) & -\frac{\mu}{\gamma} u_{3}
\end{array}\right)
$$


and clearly the partial derivatives are continuous in $\Omega \times U$. Thus by Picard's Theorem [40], it can be said that given an initial value for the problem (99), there exists a local unique solution that can be extended everywhere.

Notice, however, that our work in this thesis is concerned rather with periodic solutions $u(x)$ of (99) defined on a closed and bounded interval $x \in[0,2 L]$ with zero mean. Thus we do not expect any straightforward application of existence and uniqueness theorem to say much more about the problem at hand.

But our work in Chapters 3 to 5 in constructing leading order asymptotic solutions still provide ample details regarding the existence of such steady state periodic solutions and it would seem that with only modest modifications, these more subtle questions - though not directly within the scope of our work - can be answered. 


\section{Appendix B}

\section{Roots of the Quartic Equation}

In chapter 3, we encountered the solution of the Modified Korteweg-de Vries equation, expressed as an inverse integral involving the square root of a quartic equation. This inverse integral was later transformed to a rational expression of Jacobi elliptic functions involving the four quartic roots.

Because the details of these four roots were not necessary to the analysis, we omitted them then, but here we provide the details for the reader. Although the expressions for the roots are convoluted, they are not without theoretical merit - the behaviour of the individual roots fully describe both the non-dispersive (slow) and dispersive (fast) solutions of the $\mathrm{mKdV}$, and so much of our understanding regarding the $\mathrm{mKdV}$ can be found via an exhaustive study of the behaviour of the four roots.

The quartic equation under consideration is the following:

$$
u^{4}+\frac{6 \Delta}{\beta} u^{2}-\frac{12 C}{\beta} u+E,
$$

where all the parameters are constants. Since our equation is a depressed quartic (without a cubic term), we can directly apply Euler's Method to solve the equation (see for example, [1]).

The auxilary cubic equation is,

$$
z^{3}+\Delta z^{2}+\frac{\Delta^{2}}{4} z-E z-\frac{c^{2}}{4}
$$

The three roots of this auxilary cubic equation are, 


$$
z_{1,2,3}=\frac{\tau^{1 / 3}}{6 \beta} e^{2 \pi i k / 3}+\frac{3 \Delta^{2}+E \beta^{2}}{2 \beta \tau^{1 / 3} e^{2 \pi i k / 3}}, \quad k=0,1,2
$$

where,

$$
\begin{aligned}
\tau= & \lambda+3 \sqrt{\sigma} \\
\lambda= & 27 \Delta^{3}-27 \Delta E \beta^{2}+243 C^{2} \beta \\
\sigma= & -3\left(\frac{81 \Delta^{4} E \beta-18 \Delta^{2} E^{2} \beta^{3}+E^{3} \beta^{5}-486 \Delta^{3} C^{2}}{\beta}\right) \\
& -3\left(\frac{486 \Delta E \beta^{2} C^{2}-2187 C^{4} \beta}{\beta}\right)
\end{aligned}
$$

Now if we let,

$$
\begin{aligned}
p & =\sqrt{z_{1}} \\
q & =\sqrt{z_{2}} \\
r & =\frac{3 C}{2 \beta p q}
\end{aligned}
$$

then the four solutions of the quartic are given by,

$$
\begin{aligned}
& u_{1}=p+q+r \\
& u_{2}=p-q-r \\
& u_{3}=-p+q-r \\
& u_{4}=-p-q+r
\end{aligned}
$$

where the roots $a, b, c$, and $d$ in chapter 3 are obtained by ordering each of the roots $u_{i}$ for each encountered value of $\Delta, C$, and $E$. 


\section{Bibliography}

[1] Abramowitz, M. and Stegun, I.A. 1964 Handbook of Mathematical Functions with Formulas, Graphs, and Mathematical Tables.

[2] Amundsen, D.E., Cox, E.A., and Mortell, M.P. 2006 Asymptotic solutions for resonant sloshing of shallow water in a tank.

[3] Bender, C.M. and Orszag, S.A. 1999 Advanced Mathematical Methods for Scientists and Engineers.

[4] Blenkinsop, M.T. and Amundsen, D.E. 2006 Asymptotic Method for Approximation of Resonant Solutions of the Periodically Forced, Damped KdV Equation.

[5] Bourland, F.J. and Haberman, R. 1988 The modulated phase shift for strongly nonlinear, slowly varying, and weakly damped oscillators.

[6] Bourland, F.J. and Haberman, R. 1988 Variation of wave action: modulations of the phase shift for strongly nonlinear dispersive waves with weak dissipation.

[7] Bourland, F.J. and Haberman, R. 1991 Averaging methods for the phase shift of arbitrarily perturbed strongly nonlinear oscillators with an application to capture.

[8] Boussinesq, J. 1871 Theorie de l'intumescence liquid appelee onde solitaire ou de translation, se propageant dans un canal rectangulaire.

[9] Byrd, P.F. and Friedman, M.D. 1971 Handbook of Elliptic Integrals for Engineers and Scientists. 
[10] Chen, S.H. and Cheung, Y.K. 1995 An Elliptic Perturbation Method for Certain Strongly Non-Linear Oscillators.

[11] Chester, W. 1964 Resonant oscillations in closed tubes.

[12] Chester, W. 1968 Resonant oscillations of water waves I.

[13] Chester, W. and Bones, J.A. 1968 Resonant oscillations of water waves II.

[14] Cox, E.A. and Mortell, M.P. 1985 The evolution of resonant water-wave oscillations.

[15] Doedel, E.J. 1981 AUTO: A program for the automatic bifurcation analysis of autonomous systems.

[16] Drazin, P.G. and Johnson, R.S. 1989 Solitons: an introduction.

[17] Elias-Zunika, A. 2006 A General Solution of the Duffing Equation.

[18] Gradshteyn, I.S. and Ryzhik, I.M. 2000 Table of Integrals, Series, and Products.

[19] Grimshaw, R. 1990 Nonlinear Ordinary Differential Equations.

[20] Grimshaw, R. and Smyth, N. 1986 Resonant flow of a stratified fluid over a topography.

[21] Hagedorn, P. 1988 Non-Linear Oscillations.

[22] Kaper, B. 1976 Perturbed Nonlinear Oscillations.

[23] Kevorkian, J. and Cole, J.D. 1996 Multiple Scale and Singular Perturbation Methods.

[24] Korteweg, D.J. and de Vries, G. 1895 On the shape of form of long waves advancing in a rectangular canal, and on a new type of long stationary waves.

[25] Kuzmak, G.E. 1957 Asymptotic solutions of nonlinear second order differential equations with variable coefficients. 
[26] Luke, J.C. 1965 A perturbation method for nonlinear dispersive wave problems.

[27] Malkov, M.A. 1996 Spatial chaos in weakly dispersive and viscous media: A nonperturbative theory of the driven KdV-Burgers equation.

[28] Marchant, T.R. and Smyth, N.F. 1990 The extended Korteweg-de Vries equation and the resonant flow of a fluid over a topography.

[29] Mellville, W.K. and Helfrich, K.R. 1986 Transcritical two-layer flow over a topography.

[30] Miura, R.M. and Kruskal, M.D. 1974 Applications of a Non Linear WKB Method to the Korteweg-DeVries Equation (SIAM Journal on Applied Mathematics.)

[31] Newell, A.C. 1985 Solitons in Mathematics and Physics.

[32] Ockendon, J.R. and Ockendon, H. 1972 Resonant surface waves.

[33] Ockendon, J.R. Ockendon, H., and Johnson, A.D. 1986 Resonant sloshing in shallow water.

[34] Redekopp, L.G. and You, Z. 1995 Spatiotemporal response described by the resonantly forced modified Korteweg-de Vries equation.

[35] Rayleigh, Lord 1876 On waves.

[36] Russell, J.S. 1844 Report on waves.

[37] Smyth, N.F. 1987 Modulation theory solution for resonant flow over a topography.

[38] Struble, R.A. and Yionoulis, S.M. 1963 General Perturbational Solution of the Harmonically Forced Duffing Equation.

[39] Stoker, J, J. 1950 Nonlinear Vibrations.

[40] Walter, Wolfgang. 1991 Ordinary Differential Equations.

[41] Whitham, G.B. 2006 Linear and Nonlinear Waves. 
[42] Zabusky, N.J. and Kruskal, M.D. 1965 Interactions of 'solitons' in a collisionless plasma and the recurrence of initial states.

[43] Elias-Zuniga, A. 2006 A General Solution of the Duffing Equation. 